\title{
A Comparison of Nonlinear Filters on Mobile Robot Pose Estimation
}

\author{
by \\ Zongwen Xue B.A.Sc \\ A Thesis submitted to \\ the Faculty of Graduate Studies and Postdoctoral Affairs \\ in partial fulfilment of \\ the requirements for the degree of \\ Master of Applied Science in Electrical Engineering \\ Ottawa-Carleton Institute for Electrical and Computer Engineering \\ Department System and Computer Engineering \\ Carleton University \\ Ottawa, Ontario, Canada
}

August 2013

Copyright (C)

Zongwen Xue, 2013 
The undersigned hereby recommend to

the Faculty of Graduate Studies and Postdoctoral Affairs

acceptance of the Thesis

\title{
A Comparison of Nonlinear Filters on Mobile Robot Pose Estimation
}

\author{
Submitted by Zongwen Xue B.A.Sc \\ in partial fulfilment of the requirements for the degree of
}

Master of Applied Science in Electrical Engineering

Prof. Howard M. Schwartz, Supervisor

Professor Roshdy H.M. Hafez, Department Chair

Department System and Computer Engineering

Ottawa-Carleton Institute for Electrical and Computer Engineering Department System and Computer Engineering

Carleton University

Zongwen Xue, 2013 


\section{Abstract}

Pose estimation for mobile robots attracts a lot of attention in recent years. In order to remove process and measurement noise, a number of filtering approaches are available to use: the extended Kalman filter (EKF), the unscented Kalman filter (UKF), and several variants of the particle filter $(\mathrm{PF})$.

This thesis quantitatively explores and compares the performance of the different filtering techniques applied to mobile robot pose estimation. The main criteria compared are the magnitude of the error of pose estimation, the computational complexity, and the robustness of each filter to non-linear/non-Gaussian noise. All filters are applied on both an experimental environment of a differential wheeled robot and a simulated environment of a three-wheeled robot.

The simulation and experimental results indicate that the bootstrap particle filter has the best state estimation accuracy and the most computational cost. The UKF performs better than the EKF and they both have much less computational cost than the particle filter. 


\section{Acknowledgments}

Foremost, I would like to express my sincere gratitude to my thesis supervisor Prof. Howard Schwartz for the continuous support of my Master's study and research, for his patience, motivation, enthusiasm, and immense knowledge. His guidance helped me in all the time of research and writing of this thesis. I could not have imagined having a better advisor and mentor for my Master's study.

Also I would like to acknowledge all the professors that I have had the pleasure of studying with here at Carleton University. I would like to express my appreciation to everyone I have had the pleasure of studying with or interacting with here at Carleton.

Finally, I would like to acknowledge my mother, Liya Zhu, my wife, Junling Lu, and my daughter, Aida Xue for their unwavering support and patience during the last two years. Without their support, none of this would have been possible. 


\section{Table of Contents}

Abstract $\quad$ iii

Acknowledgments $\quad$ iv

Table of Contents $\quad$ v

List of Tables $\quad$ ix

List of Figures $\quad$ X

List of Acronyms xii

List of Symbols $\quad$ xiv

1 Introduction 1

1.1 Motivation . . . . . . . . . . . . . . . . . . 1

1.1.1 Pose Estimation for Mobile Robots . . . . . . . . . . . . . . . 1

1.1.2 Non-linear Filtering . . . . . . . . . . . . . . . . . . 3

1.2 Problem Statement . . . . . . . . . . . . . . . . 6

1.3 Thesis overview . . . . . . . . . . . . . . . . . . . . . . . . . 7

2 Filtering Theory $\quad 9$

2.1 Introduction . . . . . . . . . . . . . . . . . 9

2.2 System Modelling . . . . . . . . . . . . . . . . . . . . . . 11 
2.3 Linear Regression . . . . . . . . . . . . . . . . . . . . . . . . . . . . . 12

2.3.1 The Least Squares Estimate . . . . . . . . . . . . . . . . 12

2.3.2 Analysis of the Least Squares Estimate . . . . . . . . . . . 14

2.3.3 Recursive Identification Methods . . . . . . . . . . . . . 15

2.3.4 The Recursive Least-Squares Method (RLS) . . . . . . . . 15

2.4 The Kalman Filter . . . . . . . . . . . . . . . . . . . . . . . . . . . . 19

2.4.1 The Discrete Kalman Filter and Implementation . . . . . . . 20

2.4 .2 The Extended Kalman Filter . . . . . . . . . . . . . . . . 23

2.4 .3 Filter Parameters and Tuning . . . . . . . . . . . . . 27

2.5 Implementation Methods of the Kalman Filter . . . . . . . . . . . 32

2.5.1 Effects of Computer Roundoff on Kalman Filter Performance . 32

2.5.2 Square-Root Filtering and Factorization Methods . . . . . . . 34

2.5.3 Cholesky Decomposition Algorithm . . . . . . . . . . . 35

2.5.4 Modified Cholesky Decomposition Algorithm . . . . . . . . . 37

2.5.5 Decorrelation of Measurement Noise . . . . . . . . . . . . 37

2.5.6 Square-Root and UD Filtering Methods . . . . . . . . . . 41

2.6 The Unscented Kalman Filter . . . . . . . . . . . . . . . . . . . . . 43

2.6.1 The Unscented Transformation _. . . . . . . . . . . . . . 45

2.6.2 Implementation of the Unscented Kalman Filter . . . . . . . 47

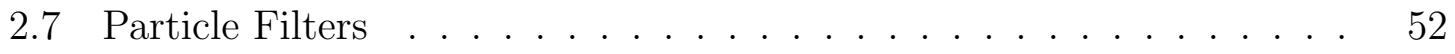

2.7.1 Monte Carlo Approach . . . . . . . . . . . . . . . . . . . 52

2.7 .2 Importance Sampling . . . . . . . . . . . . . . . . . . . 55

2.7.3 Sequential Importance Sampling . . . . . . . . . . . . . 58

2.7.4 Resampling . . . . . . . . . . . . . . . . . . 62

2.7.5 The Bootstrap Particle Filter _ . . . . . . . . . 67

2.8 Chapter Summary . . . . . . . . . . . . . . . . . . . . . . . . 68 
3 Simulation and Results for Pose Estimation

3.1 Introduction . . . . . . . . . . . . . . . . . . . . . . 72

3.2 Model Description . . . . . . . . . . . . . . . . . 73

3.2.1 System Dynamic Model . . . . . . . . . . . . . . 73

3.2.2 Measurement Model . . . . . . . . . . . . . . . . . 75

3.3 Implementation of the Filters . . . . . . . . . . . . . . . 77

3.3.1 The EKF . . . . . . . . . . . . . . . . . 77

3.3.2 The UKF . . . . . . . . . . . . . . . . . . . . . . 79

3.3.3 The Bootstrap PF . . . . . . . . . . . . . . . 80

3.3.4 Initialization . . . . . . . . . . . . . . . . 80

3.4 Results . . . . . . . . . . . . . . . . . . 81

3.5 Chapter Summary . . . . . . . . . . . . . . . . . 87

4 Experiment and Results for Pose Estimation $\quad 90$

4.1 Introduction . . . . . . . . . . . . . . . . . . . . . . . . . 90

4.2 Architecture . . . . . . . . . . . . . . . . . . . 90

4.3 The Mobile Robot and Its Actuator . . . . . . . . . . . . . . . . 94

4.4 The Sensor of the Measurement System . . . . . . . . . . . . . . 96

4.5 System Dynamics Modelling . . . . . . . . . . . . . . . . . . . . 97

4.6 Implementation of the Filters . . . . . . . . . . . . . . . . . 100

4.6.1 The EKF ......................... 100

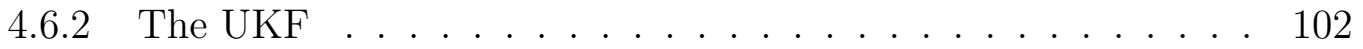

4.6.3 The Bootstrap PF . . . . . . . . . . . . . . . . . 102

4.6.4 Initialization . . . . . . . . . . . . . . . . . 102

4.7 Results . . . . . . . . . . . . . . . . . . 103

4.8 Chapter Summary . . . . . . . . . . . . . . . . . . . 110 
5 Conclusion and Recommendations

5.1 Conclusion . . . . . . . . . . . . . . . . . . . . 112

5.2 Thesis Contribution . . . . . . . . . . . . . . . . . . 114

5.3 Recommendations . . . . . . . . . . . . . . . . 115

$\begin{array}{ll}\text { List of References } & 117\end{array}$

$\begin{array}{ll}\text { Appendix A Filter Applications } & 121\end{array}$

A.1 Sample Application for the Kalman Filter . . . . . . . . . . . . . . 121

A.1.1 The System Models . . . . . . . . . . . . . . . . . . . . . 121

A.1.2 The Kalman Filter and Parameters . . . . . . . . . . . . . . . 122

A.1.3 Simulations and Results . . . . . . . . . . . . . . 123

A.2 Sample Application for the EKF, the UKF and the PF . . . . . . . 125

A.2.1 One dimensional non-linear model and parameters . . . . . . . 126

A.2.2 Simulation Results . . . . . . . . . . . . . . . . 127 


\section{List of Tables}

2.1 Solutions of Cholesky Factor for $3 \times 3$ Symmetric Matrix . . . . . . . 36

3.1 Averaged RMS Position Error in Centimeters (50 Trials) . . . . . . . 85

3.2 Averaged RMS Heading Error in Centimeters (50 Trials) . . . . . . . 85

3.3 Computational Complexity (50 Trials) . . . . . . . . . . . 86

4.1 Evaluation of Process Error . . . . . . . . . . . . . . . 95

4.2 Time-Averaged RMS Position Error in Centimeters . . . . . . . . . . 107

4.3 Time-Averaged RMS Heading Error in Centimeters . . . . . . . . . . 108

4.4 Computational Complexity . . . . . . . . . . . . . . . 109 


\section{List of Figures}

2.1 Kalman Filter Timing Diagram . . . . . . . . . . . . . . . 21

2.2 Simulation of Estimating a Constant $(R=0.01) \ldots \ldots \ldots \ldots$

2.3 Kalman Filter Simulation $(R=1)$ : Results $\ldots \ldots \ldots \ldots$

2.4 Kalman Filter $\operatorname{Simulation}(R=0.0001)$ : Results $\ldots \ldots \ldots \ldots$

2.5 Samples of MC Simulation for a Gaussian $\left(N=10^{3}\right) \ldots \ldots \ldots$

2.6 Estimated Distribution of MC Simulation for a $\operatorname{Gaussian}\left(N=10^{3}\right)$. 56

2.7 Estimated Distribution of MC Simulation for a $\operatorname{Gaussian}\left(N=10^{6}\right)$. $\quad 57$

2.8 Graphical Interpretation of Systematic Resampling . . . . . . . 66

2.9 Generic Particle Filter Flowchart _ . . . . . . . . . . . . 70

3.1 Three wheeled omni-steer mobile robot . . . . . . . . . . 73

3.2 Model of Mobile Robot . . . . . . . . . . . . . . . . . . . . . 74

3.3 Model of Measurement System . . . . . . . . . . . . . . . . 76

3.4 The real path of Mobile Robot (solid line). . . . . . . . . . 82

3.5 Performance Comparison on RMSE of Positions . . . . . . . . . 83

3.6 Performance Comparison on RMSE of Headings . . . . . . . . . . 84

3.7 RMSE on Position Estimation . . . . . . . . . . . . . . . 85

3.8 Variance of RMSE on Position Estimation . . . . . . . . . 85

3.9 RMSE on Orientation Estimation $\ldots \ldots \ldots \ldots \ldots$

3.10 Variance on Orientation Estimation . . . . . . . . . 86

3.11 Computational Complexity . . . . . . . . . . . . . . 87 
3.12 Performance Comparison on RMSE of Positions (Bootstrap PF: $N=$ $500,1000,2500) \ldots \ldots \ldots . \ldots \ldots$

4.1 The Architecture of the Experimental Platform . . . . . . . . . . . . 91

4.2 A 2-wheeled mobile robot with a front-facing LCD display . . . . . . 92

4.3 Screenshot of the OpenCV Vision System . . . . . . . . . . . . . . 93

4.4 Kinematic Model of a Differential Drive Mobile Robot. . . . . . . . . 97

4.5 Robot Navigational Path Recorded by the Camera System . . . . . . 105

4.6 Performance Comparison on RMSE of Positions . . . . . . . . . . . . 106

4.7 Performance Comparison on RMSE of Headings . . . . . . . . . . . . 107

4.8 Comparison on RMSE of Positions . . . . . . . . . . . . . . . . 108

A.1 Kalman Filter Simulation $(R=0.01)$ :Results . . . . . . . . . . . 123

A.2 Kalman Filter Simulation $(R=0.01): P_{k}^{-} \ldots \ldots \ldots 124$

A.3 Kalman Filter Simulation $(R=1)$ : Results . . . . . . . . . . . 125

A.4 Kalman Filter Simulation $(R=0.0001)$ : Results . . . . . . . . . 126

A.5 Univariate Nonstationary Growth Model(True States) . . . . . . . . . 128

A.6 Univariate Nonstationary Growth Model(True State and The EKF) . 129

A.7 Univariate Nonstationary Growth Model(True State and The UKF) • 130

A.8 Univariate Nonstationary Growth Model(True State and The PF) . . 131

A.9 Univariate Nonstationary Growth Model(Comparison) . . . . . . . 132 


\section{List of Acronyms}

\begin{tabular}{|c|c|}
\hline Acronyms & Definition \\
\hline $\mathrm{EKF}$ & Extended Kalman Filter \\
\hline UKF & Unscented Kalman Filter \\
\hline $\mathrm{PF}$ & Particle Filter \\
\hline $\mathrm{SMC}$ & Sequential Monte Carlo \\
\hline GRV & Gaussian random variable \\
\hline $\mathrm{PDF}$ & probability density function \\
\hline MCRTP & Mobile Cooperating Robot Test Platform \\
\hline RMSE & root mean square error \\
\hline SIS & sequential importance sampling \\
\hline SIR & sample importance resampling \\
\hline RLS & Recursive Least-Squares Method \\
\hline MMSE & minimum mean-square error \\
\hline $\mathrm{UT}$ & unscented transformation \\
\hline
\end{tabular}




$\begin{array}{ll}\text { MC } & \text { Monte Carlo } \\ \text { CDF } & \text { cumulative sum function } \\ \text { SDE } & \text { stochastic differential equations } \\ \text { OpenCV } & \text { Open Computer Vision Library } \\ \text { HSV } & \text { hue, saturation, value } \\ \text { APF } & \text { adaptive particle filter } \\ \text { GPU } & \text { graphics processing unit }\end{array}$




\section{List of Symbols}

\section{Symbols Definition}

\begin{tabular}{|c|c|}
\hline$k$ & time step index \\
\hline$n$ & dimension of system state vector \\
\hline$x_{k}$ & system state vector at time step $k$ \\
\hline$z_{k}$ & measurement state vector at time step $k$ \\
\hline$w_{k}$ & process noise at time step $k$ \\
\hline$\eta_{k}$ & measurement noise at time step $k$ \\
\hline$f(\cdot)$ & system function \\
\hline$h(\cdot)$ & measurement function \\
\hline $\mathcal{N}(\alpha, \beta)$ & Gaussian distribution with mean $\alpha$ and variance $\beta$ \\
\hline$p(a \mid b)$ & probability of $a$ given $b$ \\
\hline$u_{k}$ & measured control signal, i.e. system input, at time step $k$ \\
\hline$E[\cdot]$ & expected value \\
\hline & Extended Kalman Filter: \\
\hline
\end{tabular}



$Q_{k} \quad$ covariance matrix of process noise at time step $k$
$R_{k} \quad$ covariance matrix of measurement noise at time step $k$
$\phi_{k} \quad$ system transition matrix/fundamental matrix
$H \quad$ measurement sensitivity matrix
$I_{n} \quad$ identity matrix with dimension of $n$
$P_{k} \quad$ error covariance matrix of state estimate at time step $k$
$K_{k} \quad$ Kalman gain matrix at time step $k$
$\hat{x}_{k}^{-}, \hat{x}_{k} \quad$ prior and posterior state estimate at time step $k$
$\hat{P}_{k}^{-}, \hat{P}_{k} \quad$ prior and posterior error covariance matrix of state esti- mate at time step $k$
$\epsilon_{\text {roundoff }} \quad$ unit roundoff error
U unit upper triangular matrix
D diagonal matrix

\section{Unscented Kalman Filter:}

$\mathcal{X}_{i}, W_{i} \quad i$ th sigma point and its corresponding weight

$\mathcal{Y}_{i} \quad i$ th transformed sigma point

$n^{a} \quad$ dimension of augmented state vector

$x_{k}^{a}, P_{k}^{a} \quad$ augmented state vector and augmented error covariance matrix at time step $k$ 


\section{Particle Filter:}

$\hat{P}(X) \quad$ estimate of the empirical distribution

$\delta(\cdot) \quad$ Dirac delta function

$\sigma_{x} \quad$ standard deviation of $x$

$q(x) \quad$ importance sampling distribution

$N \quad$ number of samples

\section{Simulation and Experiments:}

$X_{k} \quad$ the robot system state

$x_{k}, y_{k}, \theta_{k} \quad$ Pose of the robot, i.e., Cartesian coordinates and orientation of the robot at time step $k$

$v \quad$ velocity of the robot

L distance between the rear and front wheels' axles of the robot

$\delta \quad$ angle of the steering wheel with respect to the robot longitude

$x_{L i}, y_{L i} \quad$ the Cartesian coordinates of the landmarks $i$

$r_{1}, r_{2} \quad$ measurements of the distances between the robot and landmark 1 and landmark 2 respectively

$\alpha \quad$ measurement of the angle between the landmark 3 and the mobile robot longitude 


$\begin{array}{ll}T_{s} & \text { sampling period } \\ l & \text { distance between robot's two wheels } \\ \omega & \text { angular velocity of the robot } \\ v_{L} & \text { velocity of the left wheel } \\ v_{R} & \text { velocity of the right wheel }\end{array}$




\section{Chapter 1}

\section{Introduction}

\subsection{Motivation}

\subsubsection{Pose Estimation for Mobile Robots}

Mobile robot pose estimation is a popular topic of recent research. It has been used in a great many applications ranging from underground mining to planetary exploration. Mobile robots can be found in industry, military and security environments. To accomplish complete autonomy for mobile robots in the different environments, many problems have to be solved. These problems can be defined as the combination of the three major categories: guidance, navigation, and control [1]. Guidance involves intelligent decision making of locations where a robot should go and which path or trajectory should be taken to reach the goals. Pose estimation is a robot's ability to perceive the location and orientation of where it currently is in the environment in order to ensure it is on the proper course. Finally, control of a robot is a method by which the robot can determine the signals to send to the robot's actuators.

For any mobile robot or other mobility device which has the ability to navigate in its environment, it requires the information of self-localization and representation of its local environment, i.e a map of the environment. So the problem of navigation 
is often categorized into the three fundamental components: self-localization, path planning, and map building and interpretation. Self-localization (Pose estimation) of mobile robots requires reliable real-time estimates of the system state. Normally, an accurate pose estimation is essential for a mobile robot to accomplish its tasks during navigation. However, the complete system state is not always directly measurable. Moreover, in real world applications, measurements are often corrupted by various levels of noise.

Pose estimation for a mobile robot is an ability that takes all the useful information to determine the most likely state of the mobile robot at a point in time. Mobile robot pose estimation can be used to track a robot's trajectory, enable intelligent control, and estimate its locations and orientations. In order to simulate a robot, a kinematic model which describes the underlying dynamics is needed. The robot's behavior can be modelled mathematically by a set of stochastic differential equations(SDE). Normally, the dynamic models of mobile robots have non-deterministic natures, dead reckoning is subjected to cumulative errors. Moreover, the measurements of a robot's states are often corrupted with varying degrees of error. We need algorithms to overcome these difficulties and yield reliable state estimation.

The system state of a mobile robot is generally referred to as a probability distribution that reflects the available information of the state. When new information from the system or a measurement is available, the probability distribution is updated appropriately. Since the exact system state is not always available, approximation schemes are used. Some popular examples of approximation are the Extended Kalman Filter (EKF), the unscented Kalman Filter (UKF), and the particle filter (PF) and its variants.

The main objective of this thesis is to evaluate the variations of the performance of non-linear filters applied to mobile robot pose estimation. 


\subsubsection{Non-linear Filtering}

There are a great many applications with non-linear and non-Gaussian features, including, but not limited to: localization of robots, image processing, chemical plant control, forecasting weather, and estimating noisy digital communication signals. All these applications can be classified as filtering problems which appear under of a variety of names, such as suboptimal (non-linear) filtering, stochastic filtering, Bayesian filtering and on-line inference and learning [2]. The dynamic and measurement models of non-linear systems usually have the form:

$$
\begin{aligned}
& x_{k+1}=f\left(x_{k}\right)+w_{k} \\
& z_{k+1}=h\left(x_{k+1}\right)+\eta_{k+1}
\end{aligned}
$$

where $w_{k}$ and $\eta_{k}$ are the process noise and measurement noise respectively. They are defined as zero-mean Gaussian random variables which are independent over time. The covariance matrices of the process and measurement noise are denoted by $Q_{k}$ and $R_{k}$ :

$$
Q_{k}=E\left[w_{k} w_{k}^{T}\right] \quad R_{k}=E\left[\eta_{k} \eta_{k}^{T}\right]
$$

where $E[\cdot]$ is the expectation. Therefore, $w_{k} \sim \mathcal{N}\left(0, Q_{k}\right)$ and $\eta_{k} \sim \mathcal{N}\left(0, R_{k}\right)$. The expression $\mathcal{N}(\alpha, \beta)$ denotes a Gaussian random variable(GRV) with mean $\alpha$ and variance $\beta$.

In the 1970s, the Extended Kalman Filter (EKF) was developed as an initial approach to perform state estimation for non-linear systems [3]. Since then, many non-linear filtering approaches have been developed to overcome the problems in non-linear filtering applications. The EKF, UKF and the particle filter are the three methods with more popularity. The EKF is an approximation method for non-linear state estimation problems that is based on a Taylor-series expansion to overcome the 
non-linearity in the system and measurement models.

The EKF approximates the system functions by lineazing the non-linear functions around the current estimation. At each timestep, the Jacobian of system function $f(\cdot)$ and measurement function $h(\cdot)$ are computed to obtain the local linearization of system and measurement functions. Since this analytical method approximates the true posterior state distribution by locally linearized functions, the EKF is prone to introduce errors or even diverge if the system is highly non-linear [4]. In a sample application in Appendix A.2, the EKF is applied to estimate system states of a highly non-linear model. Figure A.6 shows that the performance on the state estimate of the EKF is very poor. The estimations of the EKF are rarely close to the true state. It indicates that the local linearization method of the EKF is not sufficient to approximate the posterior density of the underlying model. Obviously, the EKF is not applicable to the state estimation of highly non-linear systems.

The Unscented Kalman filter (UKF) was proposed by S. J. Julier and J. K. Uhlmann in 1997 [5]. The UKF was developed because generally it would be more difficult to approximate a non-linear function than to approximate the state distribution of a GRV $w_{k}$ (See Eqn.(1.1) and Eqn (1.2)). The UKF algorithm implicitly captures the first and second order moments of the state distribution of a GRV [6]. Since there is no need to calculate Jacobian matrices which generally is difficult and error-prone, the UKF is easier to implement than the EKF which linearizes state dynamic functions and measurement functions [7]. The approximation of the posteriori probability density function (PDF) of the system state in the UKF is performed by a set of sigma points. These sigma points encode the mean and covariance of state distribution. The so-called sigma points are a set of deterministically chosen sample points. Each point is propagated through the nonlinear system functions and yields its corresponding transformed point. The transformed points are then used to derive the posteriori mean and covariance of the state. Finally, the posteriori state estimate 
is calculated $[6,8]$.

Although the UKF can update the state estimation by approximating the posteriori PDF of a state variable, sometimes the true PDF of the system state is nonGaussian. The analytical approaches such as the UKF and the EKF are not applicable to the non-Gaussian filtering problems. Thus they may produce large errors or diverge [5]. However, with recent advances in computational power, new methodologies for computing the posterior distributions of the system states, namely, the Sequential Monte Carlo (SMC) methods, have attracted more popularity [2]. The SMC-based methods recursively approximate the posterior PDF of the system state through Monto-Carlo integration.

The initial idea of the Monte Carlo method was first proposed in the late 1940's [9]. However, it did not gain its popularity as a practical technique in the field of filtering until a new seminal publication in 1996 by Gordon et al. [10]. Due to the widespread availability of fast and cheap computing power in the recent years, a great number of scientific papers with respect to various SMC methods and applications have been published. Variations of the SMC methods can be found under several other name definitions, including, bootstrap filters, particle filters, Monte Carlo Methods, and condensation.

The SMC methods use Monte-Carlo simulations of the underlying systems to provide a convenient approach to compute the posterior distribution. SMC methods can approximate the statistical information of the system state regardless of the linearity or Gaussianity in the system and measurement functions. Another advantage of the SMC methods is that as the number of particles increases, the variance of the approximation error decreases proportionally. Theoretically, a very large number of particles would make the approximation of the SMC methods into an identical representation of the probability density function (PDF). However it would be impractical to implement the SMC with a very large number of samples in real time applications 
due to the prohibitive computational cost. To avoid confusion, for the rest of this thesis the $\mathrm{SMC}$ is referred to the Particle Filter $(\mathrm{PF})$.

All filtering approaches described above are applied to the pose estimation problem of mobile robots models. This thesis compares and evaluates their performance, convergence, and computational complexity on the underlying system models.

\subsection{Problem Statement}

The task of this thesis is to compare and analyse the natures of non-linear filters on state estimation of the underlying mobile robot models. The non-linear filters were applied to perform robot pose estimation in a simulated environment and an experimental environment.

The simulated environment is developed which included the mobile robot's dynamic model and the measurement model. The system dynamic model of a threewheeled omni-steer mobile robot is simulated. The simulated measurement model provides the bearing and distance of the robot with respect to three known landmark.

The experimental environment that is used to evaluate the nature of the non-linear filters is the Mobile Cooperating Robot Test Platform(MCRTP). The MCRTP was developed by Professor Howard Schwartz and some undergraduate students of Carleton University. It is composed of two main components: Arduino-based two-wheeled robots, and a camera system. The mobile robots in the experimental platform are two-wheeled differential robots. The robots and the measurement system are evaluated for their model definitions. The camera system is a combination of a USB digital camera and a software application running on a computer. The camera is fixed at the ceiling on the top of experimental area. It is connected to the computer with a USB 
cable. The camera system tracks and broadcasts the robot's positions and orientations over a wireless network. In order to track the motions of the robots, the digital input to the robot motors is recorded on-line. Simultaneously, the camera system captures the robot's location and orientation in the experiments. The software that operates the robots and the camera system was revised to sample the experimental data more precisely. The non-linear filter algorithms including the EKF, the UKF and the PF were implemented on MATLAB. To perform robot pose estimation, the non-linear filters were modified with respect to the experimental environment and the simulated environment. The robots in the experiments and simulations were programmed to move along non-linear trajectories like a circular arc or a spiral. The performance of all the non-linear filters was compared and analysed, including RMSE (root mean square error) of robot pose estimation and the computational complexity.

\subsection{Thesis overview}

This chapter introduced the motivation behind the comparison of the non-linear filtering approaches. A brief introduction of state estimation and filtering techniques was given. Finally, a description of the problems and solutions was presented.

The organization of this thesis is as follows:

- Chapter 2 serves as an introduction to the background topics of modelling dynamic systems and algorithms for system state propagation. It also covers the theories and the implementation methods of the Kalman filter, the extended Kalman filter, and the unscented Kalman filter. Moreover, this chapter presents the details of sequential importance sampling (SIS), and sample importance resampling (SIR). Finally, it discusses the theories and implementations of the Bootstrap particle filter. Several examples are provided to explain the operations and theories of the particle filters. 
- Chapter 3 describes the development of the environment for the mobile robot simulation. The development includes the combination of two main components: the model of the simulated mobile robots and the sensors, and the model of the measurement system. It also introduces the implementation of the EKF, UKF and the bootstrap filters. Finally, the chapter compares the performance of non-linear filters with respect to the pose estimation. The criteria of the comparison is the RMSE of the robot pose estimation and the computational complexity.

- Chapter 4 provides a detailed description of the experimental platform including it architecture, components with underlying hardware and software. Moreover, the system dynamics model of the mobile robots is derived. It also explains how the experimental platform perform the robot localization. Specifically, the principles of operation and the data flow of the experimental system are introduced. Finally, this chapter compares the performance of the non-linear filters with respect the pose estimation of the robots. The criteria of the comparison is the RMSE of the pose estimation and the computational complexity.

- Chapter $5 \mathrm{~A}$ concise listing of the conclusion regarding the content of this thesis is given. It also recommends the possible directions of future work to further the research presented in this document. 


\section{Chapter 2}

\section{Filtering Theory}

\section{$2.1 \quad$ Introduction}

Filtering theory and, simultaneously, estimation theory is a rich and vast area. A great number of scientific papers have been published in this area. Meanwhile, many practical applications have been developed in the areas including but not limited to, control, communications and signal processing. It might be odd that the terms "filter" and "estimator" have the same meaning in the filtering field. But since the second half of the twentieth century, estimation theory has been treated as a synonym to filter theory [11].

The theory of recursive least squares estimation originated with the ambitions of two people in the early 1800s [12]. Karl Friederich Gauss completed his work on the least-squares method in 1795, but did not publish it until 1808 in his book Theoria Motvs Corporvm Celestivm [13]. Adrien Legendre developed the least-square regression theory independently while solving similar problems and published his book Nouvelles méthodes pour la détermination des orbites des cometes [14]. Both Gauss and Legendre applied their theory to estimate the locations of planets and comets based on imperfect measurements. 
In 1880 Thorvald N. Thiele, a Danish astronomer, extended the earlier leastsquares theory of his predecessor and developed a recursive algorithm similar to the Kalman filter $[15,16]$. Thiele's filter is a special case of the Kalman filter which has a scalar state, scalar measurement, and deterministic initial state. The state transitions and the measurement matrices of his filter are equal to unity [17].

There was little progress in estimation theory until the early 1940s when Kolmorgorov [18] and Wiener [19] published papers on linear minimum mean-square error estimation independently. Wiener and Kolmogorov's work in the 1940s did not arise within the context of state-space theory, but it did lay the foundation of the Kalman filter. Rudolph Kalman published his seminal paper for the discrete-time Kalman filter in 1960 [20] and one year later, Kalman and Bucy released another paper describing the continuous-time Kalman filter [21]. It is interesting to note that the most outstanding contributions to estimation theory are driven by the people from astronomical and engineering fields instead of mathematicians.

Given advancement in computing power since the second half of last century, estimation theory has been widely applied to many applications in various fields. To overcome the obstacle of non-linear filtering, the NASA Ames developed an extended version of the Kalman filter (the EKF) in the 1960s [22,23]. The EKF linearizes the non-linear system functions about a working point by applying multivariate Taylor Series expansions. In 1997, Juilier and Uhlmann introduced another extension of the Kalman filter, namely, the Unscented Kalman filter [5]. Both the extended Kalman filter and the unscented Kalman filter are suboptimal versions of the original Kalman filter.

The sequential Monte-Carlo method (SMC) has gained popularity in recent years. Although the idea of the Monte Carlo method was first proposed in the late 1940's [9], it did not become available as a practical technique until Gordon, Salmond, and Smith publish a seminal paper in 1993. This paper is known as the bootstrap filter, Novel 
approach to nonlinear/non-Gaussian Bayesian state estimation [10]. A new kind of filtering approach, the particle filter, which is also known as the bootstrap filter is proposed in this paper. Particle filtering is a technique for state estimation through a sequential Monte Carlo method of Bayesian analysis. Thousands of scientific papers have been published discussing variants and extensions to the bootstrap filter in recent years. Some of the most significant particle filter advancements after 1993 are the quasi-Monte-Carlo particle filter [24], the marginal particle filter [25], the auxiliary particle filter [26], the regularized particle filter [27] and the particle filter with RaoBlackwellization [28].

\section{$2.2 \quad$ System Modelling}

State space modelling can be divided into two main components. Generally, the system dynamics are represented by a set of differential equations. The discrete form of the differential equations specifies the propagation of state vector in time. State space variables at time step $k$ are commonly defined as $x_{k} \in \mathbb{R}^{n}$. The system dynamics model has the form

$$
x_{k}=f_{k}\left(x_{k-1}, w_{k}\right),
$$

where $f_{k}: \mathbb{R}^{n} \times \mathbb{R}^{n} \rightarrow \mathbb{R}^{n}$ is known as the system transition function. The state vector $x_{k}$ is often referred to as hidden states since they normally can not be observed directly. The process noise $w_{k} \in \mathbb{R}^{n}$ is usually assumed to be zero mean, Gaussian white noise that is independent of former and current states.

The second component of system modelling is a mathematical model describing the relationship between the states and the measurement:

$$
z_{k}=h_{k}\left(x_{k}, \eta_{k}\right),
$$


where $h_{k}: \mathbb{R}^{m} \times \mathbb{R}^{n} \rightarrow \mathbb{R}^{m}$ is the measurement function. The measurement noise $\eta_{k}$ is usually defined as a zero mean Gaussian white noise which is independent over time. Normally, the system and measurement noise are independent with respect to each other.

The rest of the chapter is dedicated to the introduction of the theories behind some of the classical and contemporary filtering methods.

\subsection{Linear Regression}

\subsubsection{The Least Squares Estimate}

This section describes the concept of linear regression. Most of the material found in this and the next sections is a summary of the books $[29,30]$. The linear regression theory can be tracked back to Gauss(1809) who used this technique to calculate the locations and the orbits of planets. The linear regression has its simplest form of parametric model:

$$
y_{i}=\varphi_{i}^{T} \theta
$$

where $y_{i}$ is a value of a measurement, $\varphi_{i}$ is an $n$-vector of known quantities. The term $\theta$ denotes an $n$-vector of unknown parameters which is also referred to as the parameter vector. We call the elements of the vector $\varphi_{i}$ regressors and $y_{i}$ the regressed variable. The variable $i$ denotes a time variable which usually takes integer values.

The model (2.3) can be extended to the multivariable case as

$$
y_{i}=\Phi_{i}^{T} \theta
$$

where $y_{i}$ is a $p$-vector and $\Phi_{i}$ denotes an $n \times p$ matrix. The unknown parameters $\theta$ is still a $n$-vector. 
The objective of the problem is to calculate an estimate $\hat{\theta}$ of the parameter vector $\theta$ from the measurements $y_{1}, \varphi_{1}, \ldots, y_{N}, \varphi_{N}$. A set of linear equations is obtained by using these measurements,

$$
\begin{aligned}
y(1) & =\varphi_{1}^{T} \theta \\
y(2) & =\varphi_{2}^{T} \theta \\
& \vdots \\
y(N) & =\varphi_{N}^{T} \theta
\end{aligned}
$$

The equations above are rewritten in a matrix form as

$$
Y=\Phi \theta
$$

where $Y=\left(y_{1}, \ldots, y_{N}\right)^{T}$ is an $N \times 1$ vector and $\Phi\left(\varphi_{1}^{T}, \ldots, \varphi_{N}^{T}\right)^{T}$ is an $N \times n$ matrix. Given the number of measurements $N=n$, the term $\Phi$ becomes a square matrix. Eqn.(2.5) can easily be solved for $\theta$ when $\Phi$ is a nonsingular matrix. However, the measurements are generally corrupted by noise and disturbances in practice. Thus, the model is redefined with some levels of errors. The prediction errors are introduced as

$$
\epsilon_{i, \theta}=y_{i}-\varphi_{i}^{T} \theta
$$

where $\epsilon$ is a $N$-vector as $\epsilon=\left(\epsilon_{1}, \ldots, \epsilon_{N}\right)^{T}$. The prediction errors $\epsilon$ are often referred to as residuals. The least squares estimate of $\theta$ is defined as the vector $\hat{\theta}$ that minimizes the loss function

$$
V(\theta, Y)=\sum_{i=1}^{N} \frac{1}{2} \epsilon_{i}^{2}=\sum_{i=1}^{N} \frac{1}{2}\left[y_{i}-\varphi_{i}^{T} \hat{\theta}\right]^{2}
$$


Given that the matrix $\Phi^{T} \Phi$ is positive definite, The loss function $V(\theta)$ has its minimum value as

$$
\hat{\theta}=\left(\Phi^{T} \Phi\right)^{-1} \Phi^{T} Y
$$

The corresponding minimal value of $V(\theta)$ is derived analytically,

$$
\min _{\theta} V(\theta)=V(\hat{\theta})=\frac{1}{2}\left[Y^{Y}-Y^{T} \Phi\left(\Phi^{T} \Phi\right)^{-1} \Phi^{T} Y\right]
$$

The least squares estimate of Eqn.(2.8) can be expressed in the equivalent form

$$
\hat{\theta}=\left[\sum_{i=1}^{N} \varphi_{i} \varphi_{i}^{T}\right]^{-1}\left[\sum_{i=1}^{N} \varphi_{i} y_{i}\right]
$$

\subsubsection{Analysis of the Least Squares Estimate}

Some statistical properties can be derived from Eqn.(2.8). Suppose that the data satisfies

$$
y_{i}=\varphi_{i}^{T} \theta_{0}+e_{i}
$$

where $\theta_{0}$ is the true parameter vector. The term $e_{i}$ is a stochastic variable with zero mean and variance $\lambda^{2}$. Eqn.(2.11) has its matrix form as

$$
Y=\Phi \theta_{0}+e
$$

where $e=\left[e_{1}, \ldots, e_{N}\right]^{T}$

Since the estimate $\hat{\theta}$ is derived from Eqn.(2.12) with its white noise $e_{i}$, it has the following properties:

1. The term $\hat{\theta}$ is an unbiased estimate of $\theta$. 
2. The covariance matrix of $\hat{\theta}$ is

$$
P(\hat{\theta})=\lambda^{2}\left(\Phi^{T} \Phi\right)^{-1}
$$

3. An unbiased estimate of $\lambda^{2}$ is given by

$$
E\left(\lambda^{2}\right)=2 V(\hat{\theta}) /(N-n)
$$

\subsubsection{Recursive Identification Methods}

The least-squares estimate is an off-line identification method. It uses all the recorded measurements simultaneously to compute the parameter estimates. The counterpart of off-line identification methods is on-line (also called recursive) identification methods. In recursive identification methods, the parameters estimates are computed recursively in time. That is, an estimate $\hat{\theta}_{i-1}$ is based upon the data up to time step $i-1$, then $\hat{\theta}_{i}$ is computed by some simple modification based on the current measurement at time $i$.

\subsubsection{The Recursive Least-Squares Method (RLS)}

Let us start from an example to explain the RLS algorithm. This example is to estimate a constant recursively. Suppose that we have a model

$$
y_{i}=b+e_{i}
$$

where $e_{i}$ denotes a white noise with zero mean and variance $\lambda^{2}$. This means that a constant is to be estimated from a number of noisy measurements. This model can 
be expressed in the form of the least squares estimate.

$$
\begin{aligned}
\varphi_{i} & =1 \quad \theta=b \\
\Phi & =(1, \cdots, 1)^{T}
\end{aligned}
$$

and the estimate $\hat{\theta}$ is computed by Eqn. 2.8 as

$$
\hat{\theta}=\left(\Phi^{T} \Phi\right)^{-1} \Phi^{T} Y=\frac{1}{k}\left[y_{1}+\ldots+y_{k}\right]
$$

So the least squares estimate $\hat{\theta}$ of $\theta$ is simply the arithmetic mean,

$$
\hat{\theta}=\frac{1}{k} \sum_{i}^{k} y_{i}
$$

This expression can be reformulated as a recursive algorithm.

$$
\begin{aligned}
\hat{\theta} & =\frac{1}{k}\left[\sum_{i-1}^{k-1} y_{i}+y_{k}\right]=\frac{1}{k}\left[(k-1) \hat{\theta}_{k-1}+y_{k}\right] \\
& =\hat{\theta}_{k-1}+\frac{1}{k}\left[y_{k}-\hat{\theta}_{k-1}\right]
\end{aligned}
$$

Eqn.(2.18) shows that the estimate of $\theta$ at time step $k$ is equal to the previous estimate plus a correction term. The correction term is proportional to the difference between the observed value $y_{k}$ at step $k$ and the predicted value $\hat{\theta}_{k-1}$ at step $k-1$. Furthermore, the prediction error is weighted by the factor $1 / k$, which means the magnitude of the changes of the estimate will decrease with the increase of the time steps.

The variance of $\hat{\theta}$ is computed by using Eqn.(2.13), neglecting a factor of $\lambda^{2}$.

$$
P_{k}=\left(\Phi^{T} \Phi\right)^{-1}=\frac{1}{k} \quad \Phi^{T}=(1, \ldots, 1)
$$


The term $1 / k$ in Eqn.(2.19) can be considered as a factor of recursion for $P_{k}$.

$$
P_{k}^{-1}=k=P_{k-1}^{-1}+1
$$

and hence

$$
P_{k}=\frac{1}{P_{k-1}^{-1}+1}=\frac{P_{k-1}}{1+P_{k-1}}
$$

\section{Derivation of the Recursive Least Squares Method}

The parameter estimate is given by Eqn.(2.10) as follows,

$$
\hat{\theta}=\left[\sum_{i=1}^{k} \varphi_{i} \varphi_{i}^{T}\right]^{-1}\left[\sum_{i=1}^{k} \varphi_{i} y_{i}\right]
$$

In order to compute Eqn.(2.22) recursively, we need to introduce the notation

$$
P_{k}=\left[\sum_{i=1}^{N} \varphi_{i} \varphi_{i}^{T}\right]^{-1}
$$

and then

$$
P_{k}^{-1}=P_{k-1}^{-1}+\varphi_{k} \varphi_{k}^{T}
$$

Eqn.(2.22) can be rewritten as the following form

$$
\begin{aligned}
\hat{\theta} & =P_{k}\left[\sum_{i=1}^{k-1} \varphi_{i} y_{i}+\varphi_{k} y_{k}\right] \\
& =P_{k}\left[P_{k-1}^{-1} \hat{\theta}_{k-1}+\varphi_{k} y_{k}\right] \\
& =\hat{\theta}_{k-1}+P_{k} \varphi_{k}\left[y_{k}-\varphi_{k}^{T} \hat{\theta}_{k-1}\right]
\end{aligned}
$$


Thus

$$
\begin{aligned}
\hat{\theta}_{k} & =\hat{\theta}_{k-1}+K_{k} \epsilon_{k} \\
K_{k} & =P_{k} \varphi_{k} \\
\epsilon_{k} & =y_{k}-\varphi_{k}^{T} \hat{\theta}_{k-1}
\end{aligned}
$$

where the term $\epsilon_{k}$ is the prediction error. It is the difference between the measured output $y_{k}$ and the prediction which is made at the time step $k-1$. The one-stepahead prediction $\hat{y}$ made at the step $k-1$ is based on the model corresponding to the estimate $\hat{\theta}_{k-1}$.

$$
\hat{y}\left(k \mid k-1 ; \hat{\theta}_{k-1}\right)=\varphi_{k}^{T} \hat{\theta}_{k-1}
$$

The vector $K_{k}$ in Eqn.(2.27) is interpreted as a gain factor showing how much the value of $\epsilon$ will modify the different elements of the parameter vector.

In order to avoid a matrix inversion at each time step, Eqn.(2.24) can be rewritten in a more useful form.

$$
P_{k}=P_{k-1}-\frac{P_{k-1} \varphi_{k} \varphi_{k}^{T} P_{k-1}}{1+\varphi_{k}^{T} P_{k-1} \varphi_{k}}
$$

The algorithm can be simplified by combining Eqn.(2.27) and (2.30).

$$
\begin{aligned}
K_{k} & =P_{k-1} \varphi_{k}-\frac{P_{k-1} \varphi_{k} \varphi_{k}^{T} P_{k-1} \varphi_{k}}{1+\varphi_{k}^{T} P_{k-1} \varphi_{k}} \\
& =\frac{P_{k-1} \varphi_{k}}{1+\varphi_{k}^{T} P_{k-1} \varphi_{k}}
\end{aligned}
$$

This form for $K_{k}$ is more convenient to use in the implementation of the RLS method since $K_{k}$ must be computed in updating $P_{k}$ at each time step.

Now the derivation of RLS method is completed. The RLS algorithm is composed of Eqn.(2.26), (2.28), (2.30), and (2.31). For convenience, the complete algorithm is 
summarized as follows:

$$
\begin{aligned}
\hat{\theta} & =\hat{\theta}_{k-1}+K_{k} \epsilon_{k} \\
\epsilon_{k} & =y_{k}-\varphi_{k}^{T} \hat{\theta}_{k-1} \\
P_{k} & =P_{k-1}-\frac{P_{k-1} \varphi_{k} \varphi_{k}^{T} P_{k-1}}{1+\varphi_{k}^{T} P_{k-1} \varphi_{k}} \\
K_{k} & =\frac{P_{k-1} \varphi_{k}}{1+\varphi_{k}^{T} P_{k-1} \varphi_{k}}
\end{aligned}
$$

We can redo the parameter estimate for the model (2.15) of the example in section 2.3.4 by using the RLS algorithm.

Since $\varphi_{k}=1$, Eqn.(2.34) becomes

$$
P_{k}=P_{k-1}-\frac{P_{k-1}^{2}}{1+P_{k-1}}=\frac{P_{k-1}}{1+P_{k-1}}
$$

which is equal to Eqn.(2.11). Thus

$$
P_{k}=K_{k}=1 / k
$$

Moreover, the parameter estimate given by Eqn.(2.32) is

$$
\hat{\theta}_{k}=\hat{\theta}_{k-1}+\frac{1}{k}\left[y_{k}-\hat{\theta}_{k-1}\right]
$$

which coincides with Eqn.(2.18).

\subsection{The Kalman Filter}

Rudolph E. Kalman published his seminal paper of the discrete-time Kalman filter (KF) in 1960 [20] and one year later, Kalman and Bucy released another paper describing the continuous-time Kalman filter [21]. The introduction of the KF is 
considered as a big advancement in the field of filtering theory. There have been thousands of experiments and literature published for this filter since then. Most of the material described in this section is a summary from the sources $[3,20,21,31,32]$.

\subsubsection{The Discrete Kalman Filter and Implementation}

The Kalman filter is a recursive predictive filter based upon a state-space model and a recursive algorithm. The state-space model represents the dynamics of the system of interest. The KF uses observations available along with system dynamic model to estimate the current system state. Generally, the discrete-time state-space model is denoted as:

$$
\begin{array}{ll}
x_{k}=\phi_{k-1} x_{k-1}+u_{k-1}+w_{k-1} & \text { Transition Function } \\
z_{k}=H_{k} x_{k}+\eta_{k} & \text { Measurement Function }
\end{array}
$$

where $x_{k} \in \mathbb{R}^{n}$ specifies the state vector and $z_{k} \in \mathbb{R}^{m}$ represents the observation vector. The term $u$ denotes the input to the system. The terms of noise $w_{k}$ and $\eta_{k}$ are the system state disturbance and the measurement error. They are normally defined as Gaussian random variables with zero mean and independent over time. The covariance matrices of the process and measurement noise are denoted by $Q_{k}$ and $R_{k}$ :

$$
Q_{k}=E\left[w_{k} w_{k}^{T}\right] \quad R_{k}=E\left[\eta_{k} \eta_{k}^{T}\right]
$$

where the noise $w_{k} \sim \mathcal{N}\left(0, Q_{k}\right)$ and $\eta_{k} \sim \mathcal{N}\left(0, R_{k}\right)$. The term $E[\cdot]$ is the expected value.

In order to implement the $\mathrm{KF}$, we assume that the initial state estimates $x_{0}$ and the estimate error covariance $P_{0}$ are available at time $k=0$. The matrices $\phi_{k}, H_{k}, Q_{k}, R_{k}$ are known for all $k=1, \ldots, n$. Furthermore, the noisy measurements 
$Z_{k}=\left\{z_{1}, \ldots, z_{k}\right\}$ are available at time step $k$.

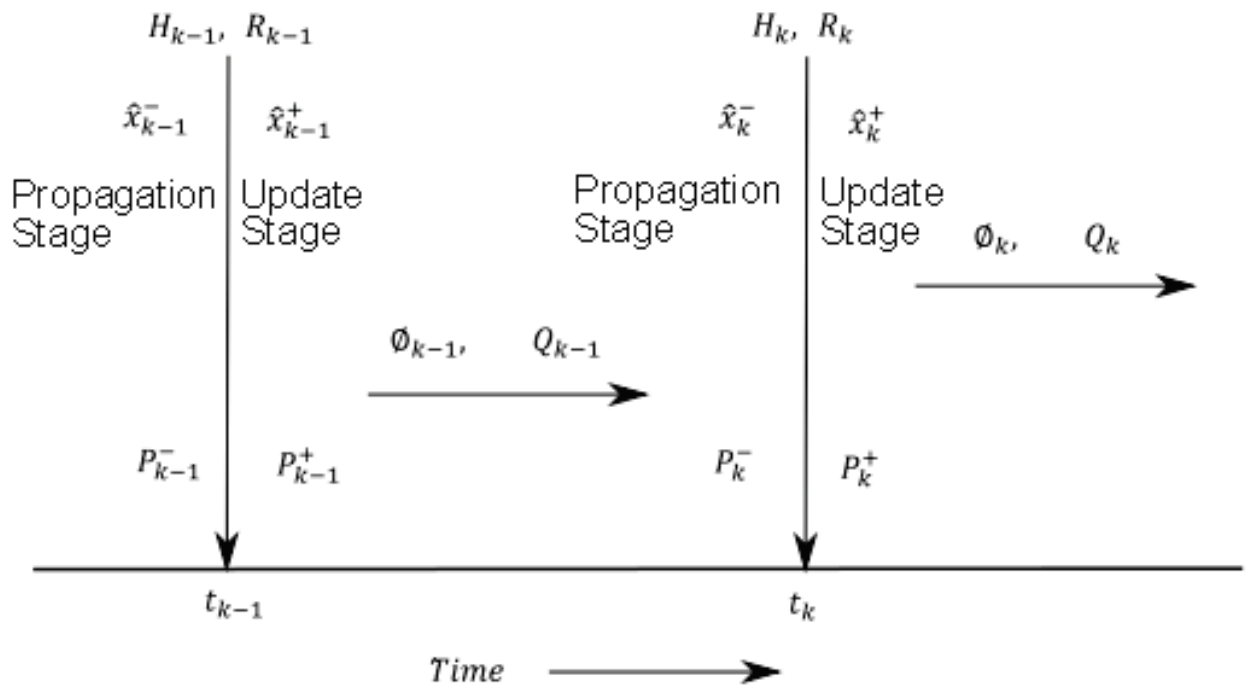

Figure 2.1: Discrete Kalman Filter Time Step Transition Diagram

The discrete KF is summarized by Eqn. (2.42) to Eqn. (2.46). The equations of the KF are a combination of two stages: the propagation stage which predicts the true state based on the previous measurement. The update stage that corrects the prediction as the most recent measurement is available. Figure 2.1 illustrates the two stages of the Kalman filter along with the time step transitions. Eqn.(2.42) and Eqn.(2.43) belong to the equations of the propagation stage,

$$
\begin{aligned}
\hat{x}_{k}^{-} & =\phi_{k} \hat{x}_{k-1}+u_{k-1} \\
P_{k}^{-} & =\phi_{k} P_{k-1} \phi_{k}^{T}+Q_{k-1}
\end{aligned}
$$

and Eqn.(2.44), (2.45), and (2.46) are the equations of the update stage.

$$
\begin{aligned}
K_{k} & =P_{k}^{-} H_{k}^{T}\left(H_{k} P_{k}^{-} H_{k}^{T}+R_{k}\right)^{-1} \\
\hat{x}_{k} & =\hat{x}_{k}^{-}+K_{k}\left(z_{k}-H_{k} \hat{x}_{k}^{-}\right) \\
P_{k} & =\left(I-K_{k} H_{k}\right) P_{k}^{-}
\end{aligned}
$$


Notice that the equations of the propagation stage compute the a priori state estimate $\hat{x}_{k}^{-}$and update the a priori error covariance estimate $P_{k}^{-}$from the time step $k-$ 1 to $k$. The equations of the update stage are applied to calculate the posterior state estimate $\hat{x}_{k}$ when the most recent measurement $z_{k}$ is available. Eqn.(2.44) computes the Kalman gain $K_{k}$. Eqn. (2.45) updates the posteriori state estimate $\hat{x}$ by introducing the correction with respect to the current measurement $z_{k}$. Finally the posteriori error covariance $P_{k}$ is updated by Eqn.(2.46).

The system function $\phi$ might change at each time step in above equations, but we generally assume that it is a constant in the practice of the KF. The process noise covariance $Q_{k}$ and the measurement noise covariance $R_{k}$ are normally considered as constants in real projects.

The propagation of state estimate $\hat{x}_{k}$ can be simplified by combining Eqn.(2.42) and $(2.45)$.

$$
\hat{x}_{k}=\phi_{k} \hat{x}_{k-1}+u_{k-1}+K_{k}\left(z_{k}-H \phi_{k} \hat{x}_{k-1}-H u_{k-1}\right)
$$

The Kalman gain $K_{k}$ is updated at each time step by the matrix Riccati equations (Eqn.(2.43),(2.44), and (2.46)) while the filter is operating. These Riccati equations are a set of recursive equations given by

$$
\begin{aligned}
P_{k}^{-} & =\phi_{k} P_{k-1} \phi_{k}^{T}+Q_{k-1} \\
K_{k} & =P_{k}^{-} H_{k}^{T}\left(H_{k} P_{k}^{-} H_{k}^{T}+R_{k}\right)^{-1} \\
P_{k} & =\left(I-K_{k} H_{k}\right) P_{k}^{-}
\end{aligned}
$$

where the covariance matrix $P_{k}$ represents the errors in the state estimates after an update of a measurement. The term $P_{k}^{-}$denotes the errors in the state estimates before an update. For the rest of this chapter, the Riccati equations are referred to as Eqn.(2.48)-(2.50). 
A simple example which illustrates the operation of the Kalman filter is presented in Appendix A.1. In this example, a scalar constant (a voltage) is estimated from noisy measurements. The measurements are corrupted by a 0.1 volt white noise. Figure A.1 shows that the filter converges eventually to the true value after the iterations. The covariance matrix $P_{k}$ approaches to zero very swiftly during the operation. It changes from the initial value of 1 to 0.0003 after 50 iterations.

The next several sections introduce the theories and the implementation methods of the extended Kalman filter (EKF). The EKF is a suboptimal filter compared to the Kalman filter and can be used to solve the problems of non-linear systems.

\subsubsection{The Extended Kalman Filter}

The Kalman filter can only work well on linear systems. In order to perform state estimation of non-linear systems, we need to use an extension of the KF, the Extended Kalman Filter (EKF). The EKF is one of the most popular approaches solving such non-linear problems. It was developed by Stanley F. Schmidt [33] and applied to the space navigation problem of the Apollo project for manned exploration of the moon. The EKF is a minimum mean-square error (MMSE) estimator. It is referred to as an extension of the Kalman filter. The EKF linearizes the non-linear system and observation functions by using Taylor series expansions. The systems are linearized about the current state estimation $\hat{x}_{k}$ in order to facilitate the estimation of non-linear systems. Notice that the implementation of the EKF needs more computational effort than the implementation of the Kalman filter. As described in section 2.4.1, the system dynamic matrix $\phi$, the observational matrix $H$, and their covariance matrices $Q$ and $R$ are constants in the implementation of the KF. Hence they can be pre-computed. This makes the Kalman filter more efficient on-line because there is no need to update those matrices at each time step. However, these matrices are impossible to be pre-computed in the EKF implementations since they are functions 
of the state and change on each time step. That is, the EKF implementations require extra computational effort to update those matrices on each time step.

The system dynamic model of a non-linear system describes the transition of statespace over time. The model is usually expressed as a set of first-order differential equations:

$$
\dot{x}=f(x, u)+w
$$

where $x$ denotes the system states. The function $f(\cdot)$ is a set of non-linear differential equations. The term $w$ defines a matrix to represent the random process noise that is a zero-mean Gaussian Random variable (GRV). Its covariance matrix can be defined as $Q=E\left[w w^{T}\right]$

It is generally not necessary to integrate Eqn.(2.51) to find explicit discrete time equations. However, we can approximate the propagation of the system states by applying numerical integration such as $4 t h$ order Runge-Kutta methods. In practice, the step size of numerical integration is adjusted with respect to required accuracy.

The measurement model is defined as

$$
z=h(x)+\eta
$$

where $\eta$ is a zero-mean random measurement noise. Its covariance matrix $R$ is denoted as $R=E\left(\eta \eta^{T}\right)$. Since the measurements are discrete in real world, the non-linear measurement function can be rewritten as:

$$
z_{k}=h\left(x_{k}\right)+\eta_{k}
$$

The non-linear function $h(\cdot)$ in Eqn.(2.53) relates the state vector $x_{k}$ and the measurement $z_{k}$. Since the elements of measurements are normally uncorrelated with each other, the discrete form of measurement noise covariance is expressed as $R=$ 
$\operatorname{diag}\left\{\eta_{1}^{2}, \ldots \eta_{m}^{2}\right\}$

In order to linearize the non-linear system and measurement functions, a first-order approximation is used for the system dynamics matrix $F$ and the measurement matrix $H$. The system functions are linearized about the current state estimate by taking the partial derivatives of the system function $f(\cdot)$ and the measurement function $h(\cdot)$. The derivations of underlying Jacobian matrices are presented in Eqn.(2.54) and (2.55).

The matrix $F$, is the Jacobian of the system dynamics matrix $f(\cdot)$ and is computed by taking the partial derivative of $f(\cdot)$ with respect to $x$,

$$
F=\left.\frac{\partial f(x)}{\partial x}\right|_{x=\hat{x}}
$$

The matrix $H$ is the Jacobian of the measurement matrix $h(\cdot)$ and is computed by taking the partial derivative of $h(\cdot)$ with respect to $x$,

$$
H=\left.\frac{\partial h(x)}{\partial x}\right|_{x=\hat{x}}
$$

In order to approximate the fundamental matrix $\phi_{k}$, we need to use the Taylorseries expansion of $e^{F T_{s}}$ to implement the fundamental matrix $\phi_{k}$ as,

$$
\phi_{k}=I+F T_{s}+\frac{F^{2} T_{s}^{2}}{2 !}+\frac{F^{3} T_{s}^{3}}{3 !}+\cdots
$$

where $T_{s}$ represents the sampling time period and $I$ is the identity matrix. Generally, the computation can be simplified by choosing the first two terms of Eqn.(2.56). The fundamental matrix has the form:

$$
\phi_{k} \approx I+F T_{s}
$$


The same equation set as the Kalman filter can be applied in applications of the EKF. Notice that we attach the subscript $k$ to the Jacobians $\phi, W, H$, and $V$ to indicate that they are different and need to be updated at each time step:

$$
\begin{aligned}
\hat{x}_{k}^{-} & =f\left(\hat{x}_{k-1}, u_{k-1}\right) \\
P_{k}^{-} & =\phi_{k} P_{k-1} \phi_{k}^{T}+Q_{k-1} \\
K_{k} & =P_{k}^{-} H_{k}^{T}\left(H_{k} P_{k}^{-} H_{K}^{T}+R_{k}\right)^{-1} \\
\hat{x}_{k} & =\hat{x}_{k}^{-}+K_{k}\left(z_{k}-h\left(\hat{x}_{k}^{-}\right)\right) \\
P_{k} & =\left(I-K_{k} H_{k}\right) P_{k}^{-}
\end{aligned}
$$

In fact, the EKF is aimed at approximating the posterior probability density function (PDF), $p\left(x_{k} \mid z_{k}\right)$ by linearizing the nonlinear system functions. Many practical applications have obtained satisfactory results by using the EKF. However, with increasing levels of system nonlinearity, the local linearity assumptions may break down, and the estimation error of the EKF is expected to increase. Appendix A.2 presents an example with a highly non-linear model. Figure A.6 shows that the estimations of the EKF is rarely close to the true state. The local linearization method of the EKF is not an adequate approach to approximate the posterior density of the underlying model. In order to linearize the highly non-linear systems, the EKFs with higher order terms of the Taylor expansion exist. However, they are not used frequently since the additional computational complexity would increase significantly.

Another issue of the EKF implementation is the difficulty of the derivation of Jacobian matrices. In many practical applications, the derivation is not trivial, and sometimes even impossible to obtain [34]. To overcome the problems of the EKF, an alternative filtering approach, the unscented Kalman filter has been developed that can achieve the performance of a second order of state distribution without the need of Jacobian derivation. 


\subsubsection{Filter Parameters and Tuning}

The parameters of the Kalman filter and the EKF can be tuned to achieve desired performance of state estimation. The measurement noise covariance $R$ and the process noise covariance $Q$ should be determined appropriately in the Kalman filter's implementations. It is relatively easy to determine the measurement noise. In practice, the measurement noise covariance can usually be measured prior to operations of the filter [32]. By taking some off-line measurements, the variance of the measurement noise can be determined. However, the process noise covariance is more difficult to assess since the state of a process being estimated is generally not directly observable.

The performance of the filter can be tuned by adjusting the process error covariance matrix $Q$. A reasonable output of the state vector produced by the Kalman filters or the EKF can be a criteria to judge the behaviour of a filter. Moreover, the estimates cannot be too noisy, that is, the variance of the state vector has to be reasonable. In practical applications, the measurement noise plays a major role in introducing noise into estimates. The objective of tuning $Q$ is to reduce the magnitude of noise in state estimate. As shown in Eqn.(2.43), a larger $Q$ can produce a larger a priori error covariance $P_{k}^{-}$. A larger $P_{k}^{-}$would lead to a larger Kalman gain $K$ as indicated in Eqn.(2.44). The term $e$ in Eqn.(2.63) reflects the measurement innovation. Since the measurement noise $\eta$ is a term of the innovation process $e$ as shown in Eqn.(2.65) and $K_{k}(e)$ is a term to calculate state estimate $\hat{x}$, a larger $K$ would cause more measurement noise to be added to the a posteriori state estimate $\hat{x}$.

$$
\begin{aligned}
& \hat{x}_{k}=\hat{x}_{k}^{-}+K_{k}(e) \\
& \hat{x}_{k}=\hat{x}_{k}^{-}+K_{k}\left(z_{k}-h\left(\hat{x}_{k}^{-}\right)\right) \\
& \hat{x}_{k}=\hat{x}_{k}^{-}+K_{k}\left(h_{k}\left(x_{k}\right)+\eta-h\left(\hat{x}_{k}^{-}\right)\right)
\end{aligned}
$$


So, the main tuning rule is to select $Q$ as large as possible without the state estimates becoming too noisy. Since each element of the process noise is typically assumed to act on its respective state independently, the process noise covariance $Q$ can be simplified to a diagonal matrix: $\operatorname{diag}\left(w_{1}^{2}, \ldots, w_{n}^{2}\right)$. Finally, each of the diagonal elements can be adjusted independently to fine tune each element of $Q$ individually.

A simple example is presented in Appendix A.1 to illustrate the impact of white noise component which is added to the observations. This example is a simulation to the real life situation of estimating a voltage from the noisy measurements. The system model is

$$
x_{k}=x_{k-1}+w_{k}
$$

where $w_{k} \sim \mathcal{N}\left(0,0.001^{2}\right)$. The measurement model is

$$
z_{k}=x_{k}+\eta_{k}
$$

where $\eta_{k} \sim \mathcal{N}\left(0,0.1^{2}\right)$. The process noise $w_{k}$ is relatively small, thus the process noise variance is set to $Q=1 e-5$. However the measurement noise variance $R$ is not negligible since the measurement noise is substantial.

Figure 2.2-2.4 illustrate the results of the simulations with respect to the different values of $R$. The random constant $x=-0.37727$ is depicted by the solid line, the noisy measurement is given by the cross marks, and the filter estimate by the remaining curve.

In the first simulation, the measurements are corrupted by a 0.1 volt white noise. The measurement variance is set at $R=(0.1)^{2}=0.01$. Figure 2.2 shows that the filter converges eventually to the true value after the iterations.

Two more simulations are conducted with the different values of $R$. The covariance $R$ is increased or decreased by a factor of 100 respectively. The different performance indicates that $R$ can be a factor affecting the speed of filter's convergence. 


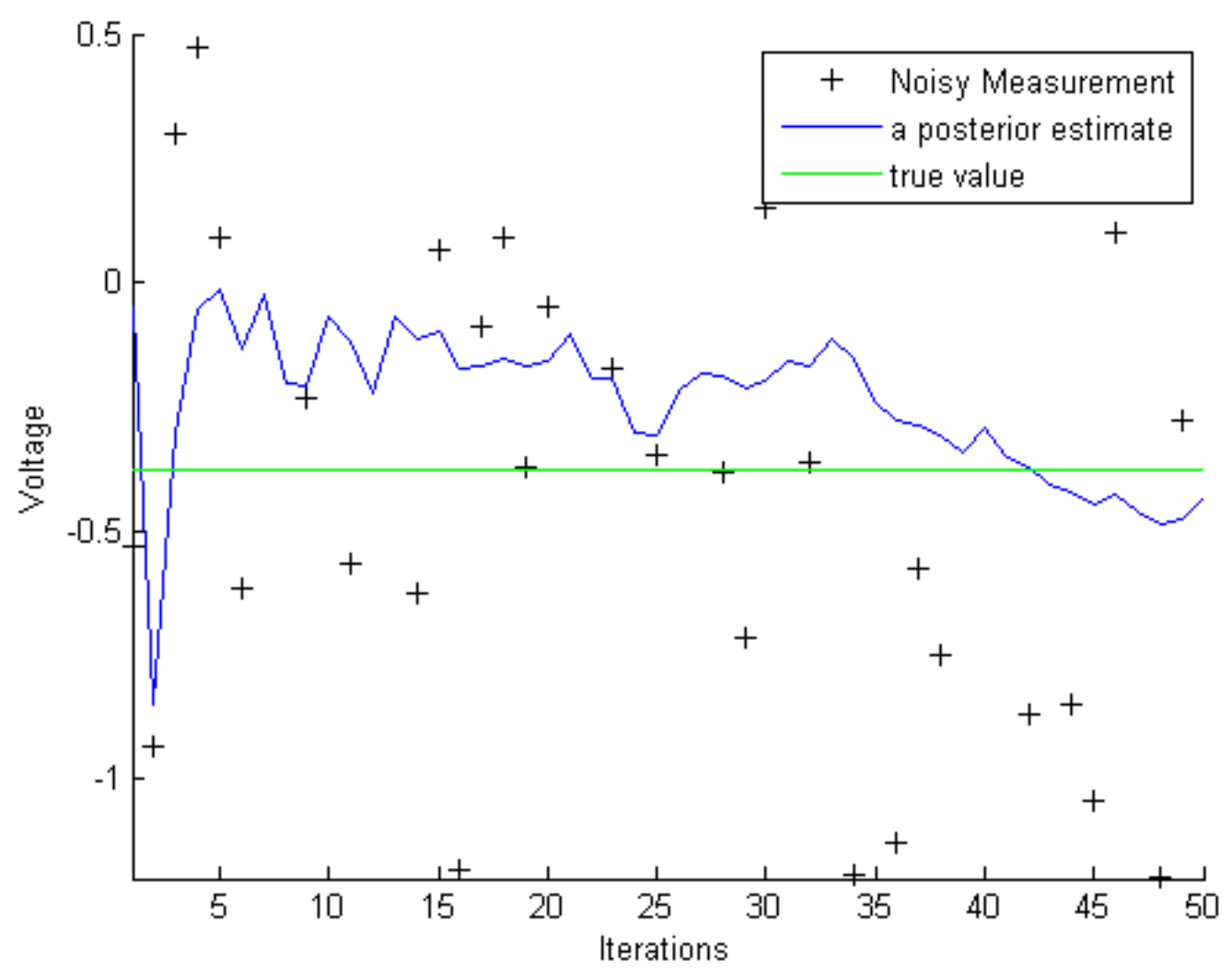

Figure 2.2: The first simulation: $R=0.1^{2}=0.01$.

As shown in Figure 2.3, as the measurement variance was 100 times greater (i.e. $R=1$ ), the filter converges more slowly but it has a smoother performance. Alternatively, as the measurement variance is set 100 times smaller(i.e. $R=0.0001$ ), the filter tends to trust the noisy measurement so it has a larger variance as shown in Figure 2.4,

This example clearly demonstrates the nature of the Kalman filter with respect to the different measurement noise covariance $R$. The filter responds slower to the noisy measurements when $R$ is set 100-time larger. Alternatively, in the simulation where $R$ is decreased by a factor of 100 , the filter responds much quicker as shown in Figure 2.4.

It is also necessary to note that the magnitude of the measurement error does not 


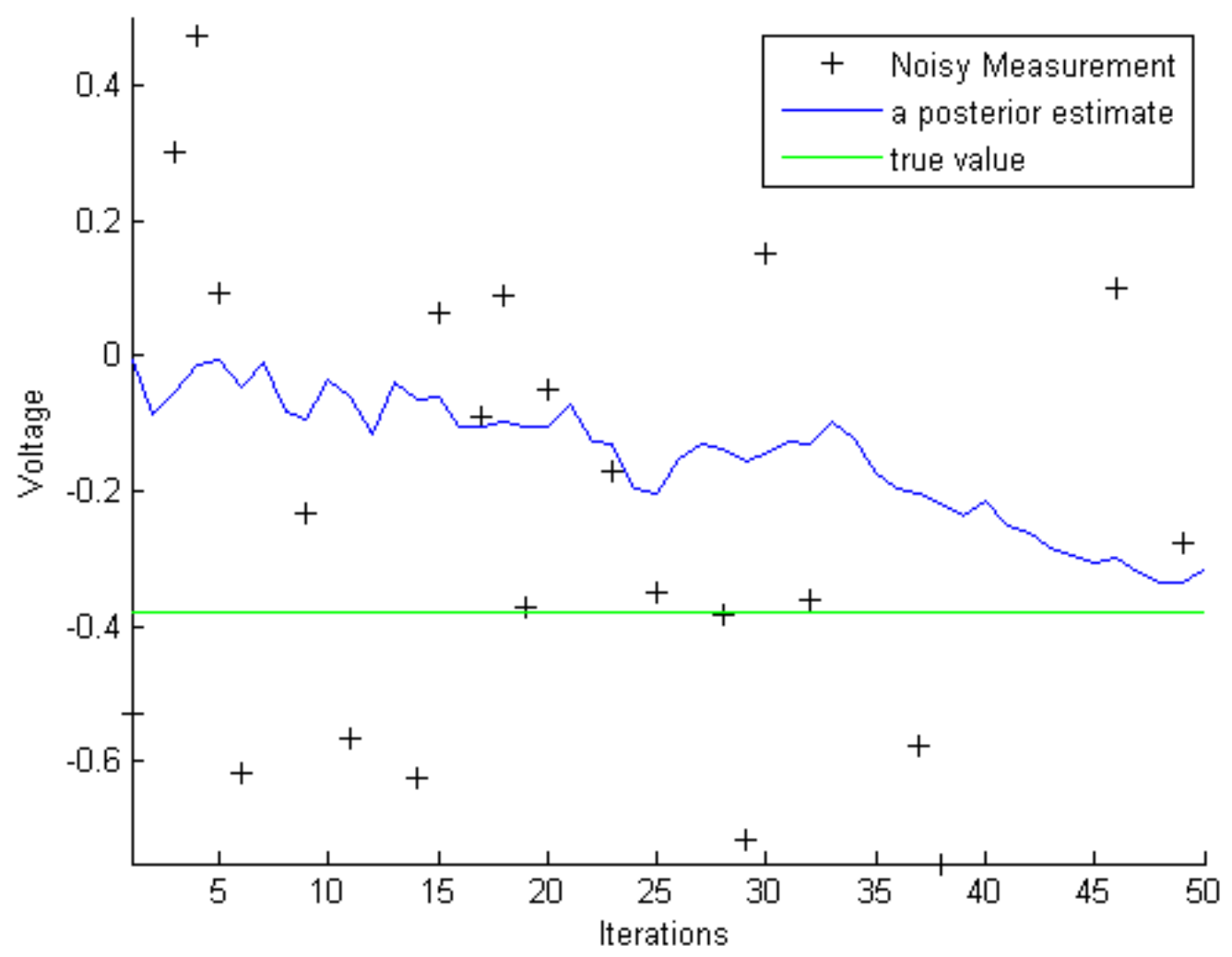

Figure 2.3: Simulation: $R=1$. The filter is slower in convergence, resulting from the reduced estimate variance.

remain constant in most cases. For example, the measurement system used in the experiment in Chapter 4 is a USB camera set (Logitech HDc615). In order to estimate the measurement error generated by the camera system, we conducted experiments by comparing the real measurement by a ruler with the readings of pixels from a camera system. The maximum error produced in the experiments is measured and calculated in $x$ and $y$ direction. Based on our experiments, the maximum error in $x$-direction is about \pm 9 pixels (corresponding to $3.5 \mathrm{~cm}$ ). Whereas the maximum error in $\mathrm{y}$-direction is about \pm 3 pixels (corresponding to $1.2 \mathrm{~cm}$ ). That is, the magnitude of the error in the measurements of a robot movements would be different with respect to the directions of the robot's movements ( $x$-direction or $y$-direction). 


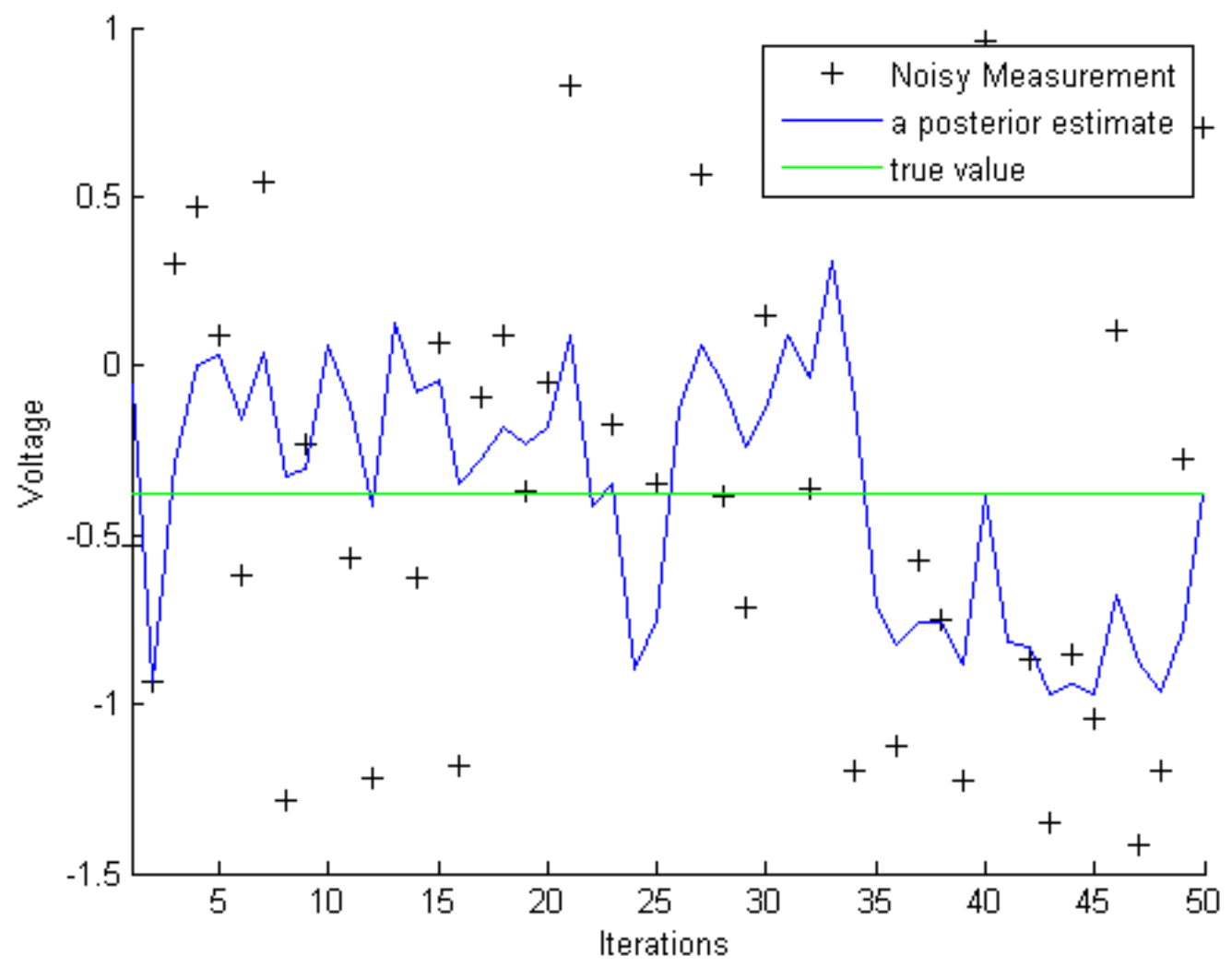

Figure 2.4: Simulation: $R=0.00011$. The filter has a noisy performance, resulting from the increased estimate variance.

The process noise $Q$ can also change on-line during the experiment. The matrix $Q_{k}$ is normally to be adjusted on the basis of different dynamics. For example, in the case of tracking a mobile robot trajectory, we might reduce the magnitude of $Q_{k}$ if the robot is moving slowly, and increase the magnitude if the robot starts moving rapidly since wheel-slipping may occur more often when a robot moves rapidly. In such cases, the determination of $Q_{k}$ is based on both uncertainty of the system model and uncertainty about the change of environment. 


\subsection{Implementation Methods of the Kalman Filter}

Up to now, we have discussed the discrete Kalman filter, the Extended Kalman filter and their parameters' tuning. As described in the last section, the performance of the Kalman filter or the EKF can be tuned by adjusting the covariance matrix of estimation uncertainty.

During the first implementations of the Kalman filters in 1960s, the observed mean-squared estimation errors were often much larger than their theoretical values which is indicated by the covariance matrix. It was noticed that the variance of the estimation errors diverged from their theoretical value. Moreover, the solutions of the Riccati equations (Eqn.(2.43),(2.44), and (2.46)) were observed to have negative variances which is impossible in theory. Finally, the problem was found out to be caused by computer roundoff and some alternative implementation methods were developed to deal with it.

The content in the next section concerning the implementation methods of the Kalman filter is a summary of the sources [17,35-37].

\subsubsection{Effects of Computer Roundoff on Kalman Filter Per- formance}

Computer roundoff is a characteristic in floating-point arithmetic to indicate the machine precision. It is simply expressed as a single parameter $\epsilon_{\text {roundoff }}$ which is called the unit roundoff error:

$$
1+\epsilon_{\text {roundoff }} \equiv 1 \text { in machine precison }
$$

The computer roundoff problem was encountered when the Kalman filter was first implemented to accomplish a mission in the Apollo moon program in the 1960s. Finally, 
the problem was solved satisfactorily by finding new implementation methods. The roundoff problems occurred more often with shorter word-lengths and less accurate implementations of bit-level arithmetic than current microprocessors with the current ANSI/IEEE standard.

However, roundoff can still be a problem when the Kalman filter is implemented in some real-time applications or on embedded computers where computational resources are limited. The next example is modified from [38], and regards an illconditioned roundoff problem during the filter implementation.

Let $I_{n}$ denotes the $n \times n$ identity matrix. Suppose we have a measurement sensitivity matrix:

$$
\begin{gathered}
H=\left[\begin{array}{ccc}
1 & 1 & 1 \\
1 & 1 & 1+\delta
\end{array}\right] \\
P_{0}=I_{3} \quad R=\delta^{2} I_{2}
\end{gathered}
$$

where $\delta^{2}<\epsilon_{\text {roundoff }}$ and $\delta>\epsilon_{\text {roundoff }}$. The product of $H P_{0} H^{T}$ with roundoff is equal to:

$$
H P_{0} H^{T}=\left[\begin{array}{cc}
3 & 3+\delta \\
3+\delta & 3+2 \delta
\end{array}\right]
$$

where $H$ is singular. The result of $R+H P_{0} H^{T}$ is also a singular matrix. In this case, the filter measurement update will fail since the matrix $R+H P_{0} H^{T}$ is singular and not invertible.

Ill-Conditioned and Well-Conditioned Problems are terms to describe the sensitivity regarding the input data and the solution method. A Well-Conditioned problem 
is a condition such that the solution is sensitive to the input data. An ill-Conditioned problem occurs when the solution is insensitive to the input data. Due to the uncertainty of the input data and the numerical precision in the implementation, a matrix $A$, for example can be called ill-conditioned with respect to inversion if $A$ is close to being singular.

During the Kalman filtering implementation, the following factors may contribute to the cause of ill-conditioning problems:

- Large uncertainties in the values of the matrix $\phi, Q, H$, or $R$ due to modelling errors.

- Large ranges of actual values of the matrix parameters, the measurements, or the state values due to poor choices of dimension units or scaling.

- Failure to calculate the Kalman gain since the intermediate result $R=H P H^{T}+$ $R$ is ill-conditioned for inversion as shown in the above example.

\subsubsection{Square-Root Filtering and Factorization Methods}

The square-root filters are the most reliable and numerically stable methods for Kalman filtering implementations. The methods reformulate the matrix Riccati equations to yield certain factors of the covariance matrix $P$. These factors are much better conditioned for matrix operations than $P$ itself. Thus, the filter implementations using these factors are more robust when dealing with the roundoff errors.

James E. Potter developed the first factorization method to transform the Riccati equations when he was a graduate student in mathematics at MIT in 1962. Potter found that a positive-definite matrix can be transformed into a product of its matrix square roots and a Cholesky factor of the covariance matrix can be used for the measurement update. Potter's innovation is called square-root filtering. The SquareRoot filter was implemented on the Apollo computer and successfully accomplished 
all the Apollo missions.

The Square-Root Filtering methods are useful in Kalman filtering implementation in the following ways:

- Decompose the covariance matrices $(R, Q$, and $P)$ in order to implement squareroot filters.

- Decorrelate measurement errors between elements of vector-valued measurements. The element can be processed sequentially as independent scalar measurements after decorrelation.

- Be part of a numerically stable method to compute the matrix inversion $\left(H P H^{T}+R\right)^{-1}$, the intermediate result of the conventional Kalman filter.

\subsubsection{Cholesky Decomposition Algorithm}

The product of a matrix $C$ with its own transpose in the form $C C^{T}=M$ gives a symmetric product of $C$. The term $C$ is called a Cholesky factor of $M$. The Cholesky decomposition algorithm is a method of generating a triangular Cholesky factor from a symmetric, nonnegative definite matrix. It solves the equation $P=C C^{T}$ for a triangular matrix $C$ by using Cholesky decomposition, given the matrix $P$. The following is an example of the Cholesky algorithm finding a lower triangular Cholesky 
factor $P=C C^{T}$ of symmetric $3 \times 3$ matrix $P$ :

$$
\begin{aligned}
{\left[\begin{array}{lll}
p_{11} & p_{21} & p_{31} \\
p_{21} & p_{22} & p_{32} \\
p_{31} & p_{32} & p_{33}
\end{array}\right] } & =\left[\begin{array}{ccc}
c_{11} & 0 & 0 \\
c_{21} & c_{22} & 0 \\
c_{31} & c_{32} & c_{33}
\end{array}\right]\left[\begin{array}{ccc}
c_{11} & 0 & 0 \\
c_{21} & c_{22} & 0 \\
c_{31} & c_{32} & c_{33}
\end{array}\right]^{T} \\
& =\left[\begin{array}{ccc}
c_{11}^{2} & c_{11} c_{21} & c_{11} c_{31} \\
c_{11} c_{21} & c_{c 21}^{2}+c_{22}^{2} & c_{21} c_{31}+c_{22} c_{32} \\
c_{11} c_{31} & c_{21} c_{31}+c_{22} c_{32} & c_{31}^{2}+c_{32}^{2}+c_{33}^{2}
\end{array}\right]
\end{aligned}
$$

The corresponding matrix elements of $P$ and $C$ can be equated as six equations (only six element are independent due to symmetry). The solutions for elements in $C$ are given in Table 2.1.

Table 2.1: Solutions of Cholesky Factor for $3 \times 3$ Symmetric Matrix

\begin{tabular}{ll}
\hline Six Scalar Equations of $P$ & Solutions for element inC \\
\hline$p_{11}=c_{11}^{2}$ & $c_{11}=\sqrt{p_{11}}$ \\
$p_{21}=c_{11} c_{21}$ & $c_{21}=p_{21} / c_{11}$ \\
$p_{22}=c_{21}^{2}+c_{22}$ & $c_{22}=\sqrt{p_{22}-c_{21}^{2}}$ \\
$p_{31}=c_{11} c_{31}$ & $c_{31}=p_{31} / c_{11}$ \\
$p_{32}=c_{21} c_{31}+c_{22} c_{32}$ & $c_{32}=\left(p_{32}-c_{21} c_{31}\right) / c_{22}$ \\
$p_{33}=c_{31}^{2}+c_{32}^{2}+c_{33}^{2}$ & $c_{33}=\sqrt{p_{33}-c_{31}^{2}-c_{32}^{2}}$ \\
\hline
\end{tabular}

A general solution of Cholesky decomposition is given in Algorithms 2.1 by looping through the rows and columns of Cholesky factor $C$ with the prior results. 


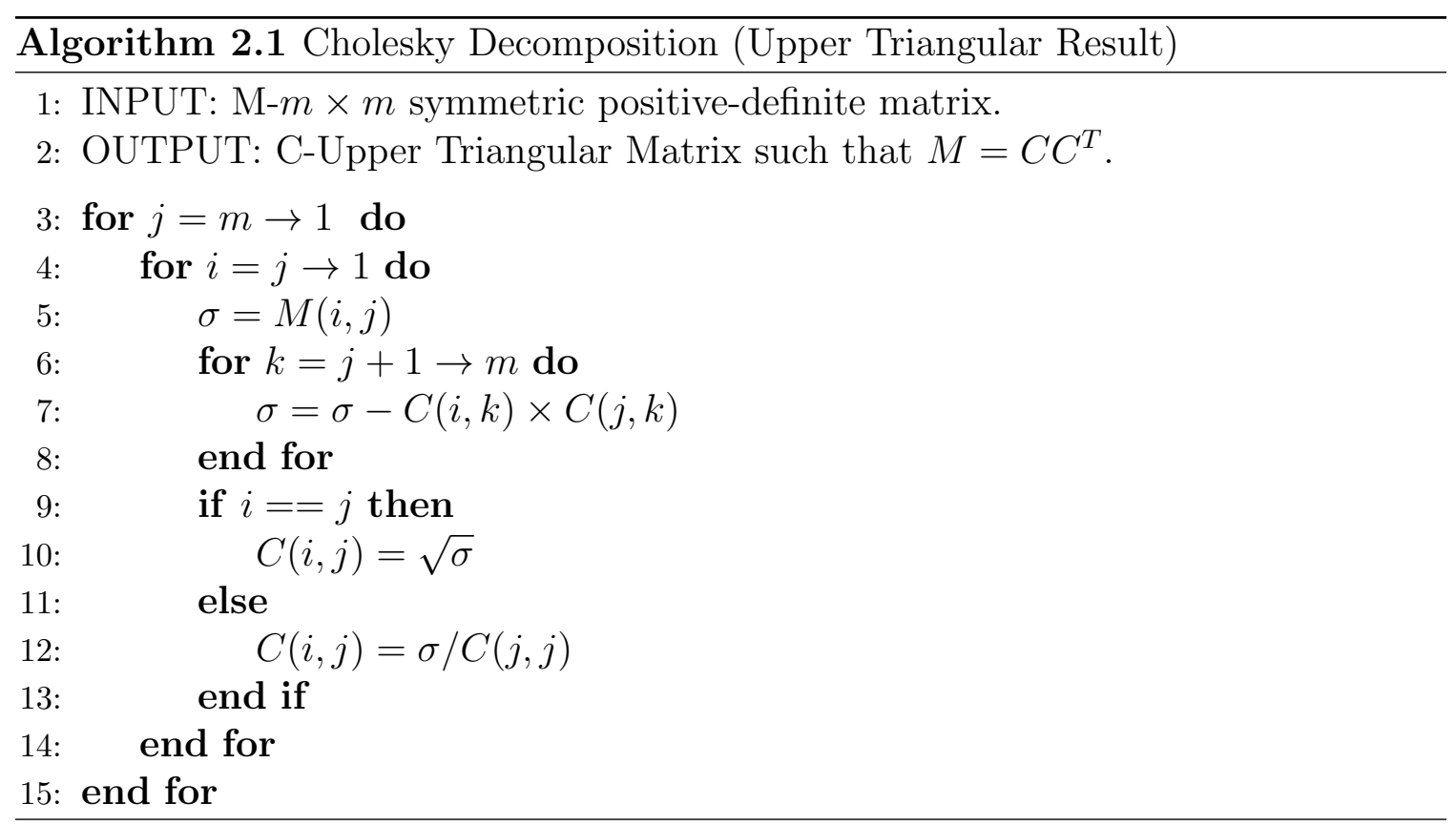

\subsubsection{Modified Cholesky Decomposition Algorithm}

In the 1990s, a simpler algorithm than the symmetric Cholesky decomposition was developed for computing the triangular Cholesky factor. It is called the modified Cholesky decomposition. The modified algorithm decomposes the positive-definite matrix $M$ into a product $M=U D U^{T}$ such that $U$ is unit upper triangular and $D$ is diagonal. This method is also called the UD decomposition algorithm.

An implementation of $U D$ decomposition is illustrated in Algorithm 2.2. It takes $M$ as the input and $U$ and $D$ as the outputs. This algorithm is quite similar to the Cholesky decomposition with one notable exception: the modified Cholesky decomposition does not require taking square roots.

\subsubsection{Decorrelation of Measurement Noise}

The decomposition algorithms can also be used to decorrelate the covariance matrix of measurement uncertainty $R$. The measurement vector $z$ is reformatted such that the measurement errors are uncorrelated from element to element. The decomposition 


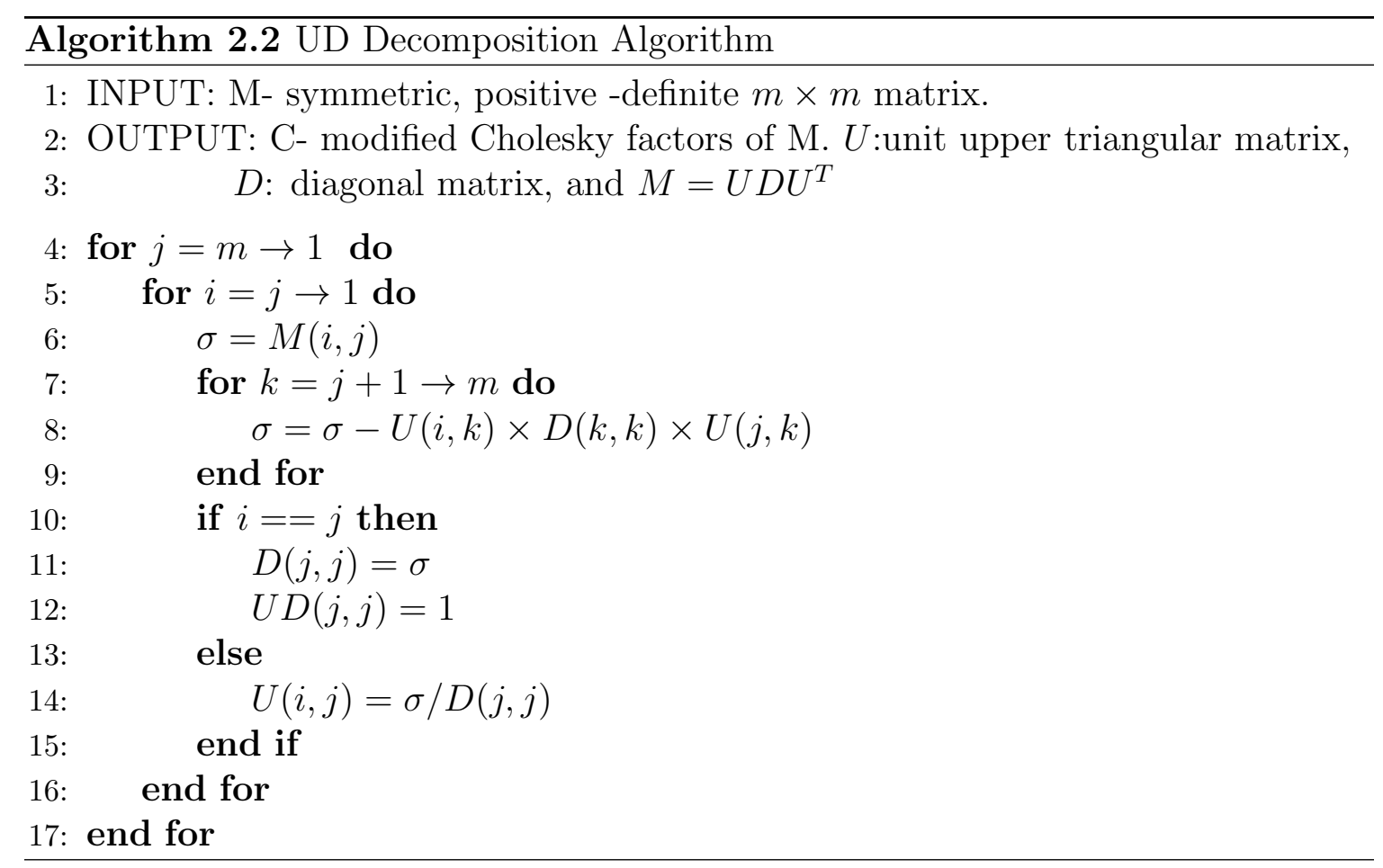

generates a new covariance matrix of measurement uncertainty which is a diagonal matrix. The elements of the reformatted matrix vector $\tilde{z}$ can thus be processed sequentially as uncorrelated scalar measurements.

Given a measurement function:

$$
z=H x+\eta
$$

where $H$ is measurement sensitivity matrix and the elements of measurement noise $\eta$ are correlated. Its covariance matrix is denoted as,

$$
E\left(\eta \eta^{T}\right)=R
$$

Since $R$ is not a diagonal matrix, the scalar component of $z$ can not be processed sequentially as a scalar observation along with its independent measurement error. However, the covariance matrix $R$ can be factored by using the modified Cholesky 
decomposition method as:

$$
R=U D U^{T}
$$

where $D$ is a diagonal matrix and $U$ is a unit upper triangular matrix. Since a unit upper triangular matrix is always non-singular, it always has a matrix inverse. Furthermore, the inverse of a unit upper triangular matrix is still a unit upper triangular matrix.

The measurement vector is redefined as:

$$
\begin{aligned}
\tilde{z} & =U^{-1} z \\
& =U^{-1}(H x+\eta) \\
& =\left(U^{-1} H\right) x+\left(U^{-1} \eta\right) \\
& =\tilde{H} x+\tilde{\eta}
\end{aligned}
$$

The new measurement sensitivity matrix is $\tilde{H}=U^{-1} H$ and the new measurement

error is denoted as $\tilde{\eta}=U^{-1} \eta$. The new covariance matrix $\tilde{R}$ of the measurement errors $\tilde{\eta}$ is restructured as,

$$
\begin{aligned}
\tilde{R} & =E\left(\tilde{\eta} \tilde{\eta}^{T}\right) \\
& =E\left(\left(U^{-1} \eta\right)\left(U^{-1} \eta\right)^{T}\right) \\
& =E\left(U^{-1} \eta \eta^{T} U^{-1 T}\right) \\
& =U^{-1} E\left(\eta \eta^{T}\right) U^{-1 T} \\
& =U^{-1} R U^{-1 T} \\
& =U^{-1}\left(U D U^{T}\right) U^{-1 T} \\
& =D
\end{aligned}
$$

where $\tilde{R}$ is a diagonal covariance matrix. It indicates that the redefined measurement has uncorrelated elements of measurement noise. 
The next step is to solve the unit upper triangular systems of equations

$$
\begin{gathered}
U \tilde{z}=z \\
U \tilde{H}=H
\end{gathered}
$$

for $\tilde{z}$ and $\tilde{H}$, given $z, H$, and $U$. It is not necessary to invert $U$ to solve for $\tilde{z}$ and $\tilde{H}$. It can be done by a method called back substitution. The algorithm 2.3 performs the task of back substitution.

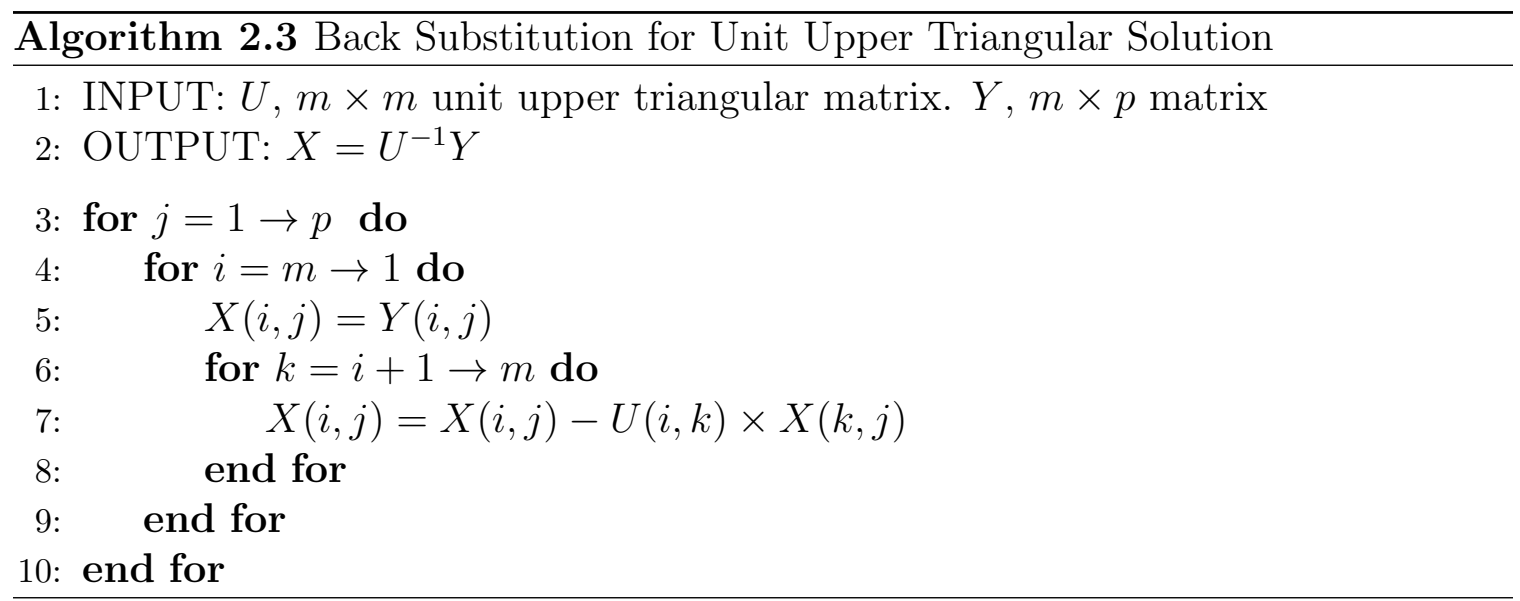

A complete procedure to perform measurement decorrelation is presented in Algorithm 2.4.

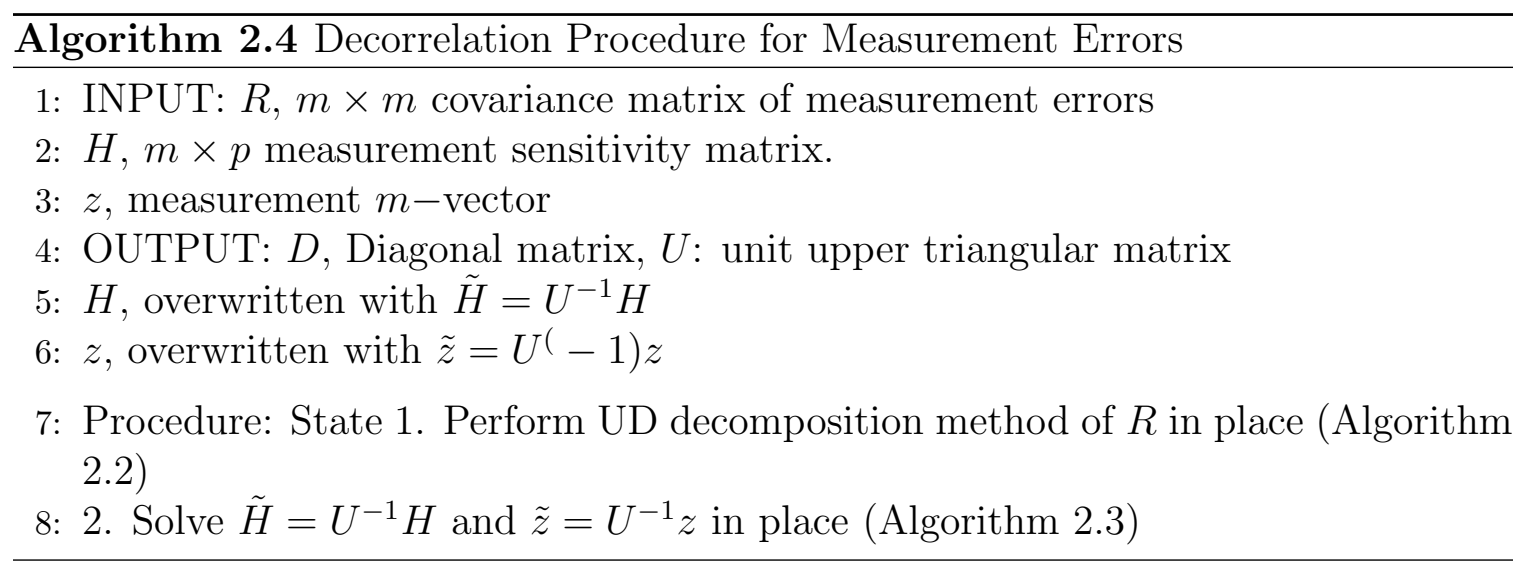

We summarize the procedure of measurement decorrelation here. 
- Perform $U D$ decomposition with respect to $R$ as $R=U D U^{T}, R$ is replaced by $D$.

- Solve for $\tilde{H}=U^{-1} H$ and $\tilde{z}=U^{-1} z$.

- Transform the measurement vector $z=H x+\eta$ with the correlated measurement errors $E\left(\eta \eta^{T}\right)=R$ into the reformatted measurement $\tilde{z}=\tilde{H}+\tilde{\eta}$ with uncorrelated measurement errors $\tilde{\eta}$ and $E\left(\tilde{\eta} \tilde{\eta}^{T}\right)=D$ (diagonal matrix)

\subsubsection{Square-Root and UD Filtering Methods}

Square-root Kalman filters reformulate the Riccati equations by using Cholesky decomposition or modified Cholesky decomposition of the state estimate error covariance $P$. This section presents the two most popular forms of square-root filtering methods.

1. Carlson-Schmidt square-root filtering using Cholesky factors of $P$.

2. Bierman-Thornton $U D$ filtering using modified Cholesky factors of $P$.

\section{Carlson-Schmidt Square-Root Filtering}

This filtering method is composed of a pair of algorithms, including an algorithm for the propagation stage of the $\mathrm{KF}$ and an algorithm for the update stage. If the covariance matrices $R$ and $Q$ are not diagonal matrices, Cholesky decomposition is required to factorize these matrices. The Carlson Triangular Measurement Update is presented in Algorithm 2.5, showing a complete procedure which updates the measurements sequentially.

\section{Bierman-Thornton UD Filtering}

This method is also composed of a pair of algorithms as 


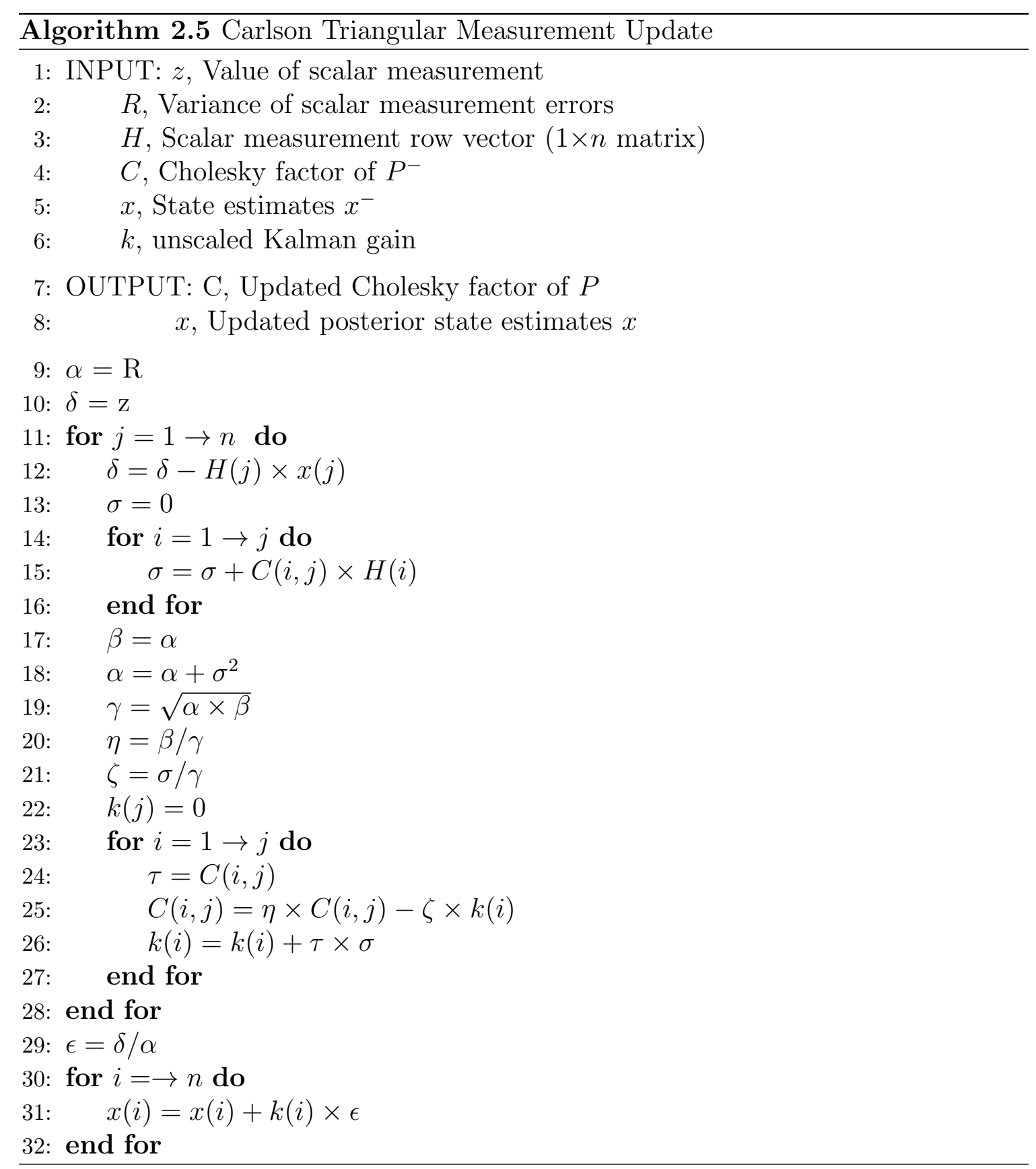

- The Thornton algorithm for the propagation stage that updates $U$ and $D$

- The Bierman algorithm for the update stage that calculates the modified Cholesky factors $U$ and $D$ of the covariance matrix $P=U D U^{T}$ 
Bierman's algorithm is presented in Algorithm 2.6. It is one of the most stable implementations of the Kalman filter measurement update. The algorithm was developed by Gerald J. Bierman. It is similar to the Carlson algorithm but avoids taking scalar square roots.

The Bierman-Thornton UD filtering approach is implemented in Matlab and $\mathrm{C}++$ to perform the mobile robot's pose estimation in the simulations of Chapter 3 and the experiment of Chapter 4.

\subsection{The Unscented Kalman Filter}

As mentioned earlier in Sec.2.4.2, although many practical applications have obtained satisfactory results by using the EKF, there are still some problems that exist. First, with increasing levels of system nonlinearity, the estimation error of the EKF is expected to increase. Moreover, the derivation and the calculation of the Jacobian matrices during the EKF implementation are sometimes difficult and error-prone. In order to overcome problems of the EKF, Jaulier and Uhlmann introduced the Unscented Kalman filter (UKF) in 1997 to approximate the nonlinearities [5]. Like the $\mathrm{EKF}$, the UKF is also a sub-optimal recursive MMSE (minimum mean square error) estimator and it is specific for nonlinear systems. The UKF avoids using Jacobian matrices to linearize the nonlinear system and measurement models. Instead, the UKF uses a method referred to as the unscented transformation (UT) to estimate the distribution of state variables. A set of sample points (sigma points) are deterministically selected by the UT. These sample points are then propagated through the true non-linear system functions and a set of transformed sample points are generated. Finally, the propagated mean and covariance of the transformed sample points are calculated to yield the posterior state estimates. The UKF is based on the assumption that it is easier to approximate a PDF than an arbitrary nonlinear function [5]. The 


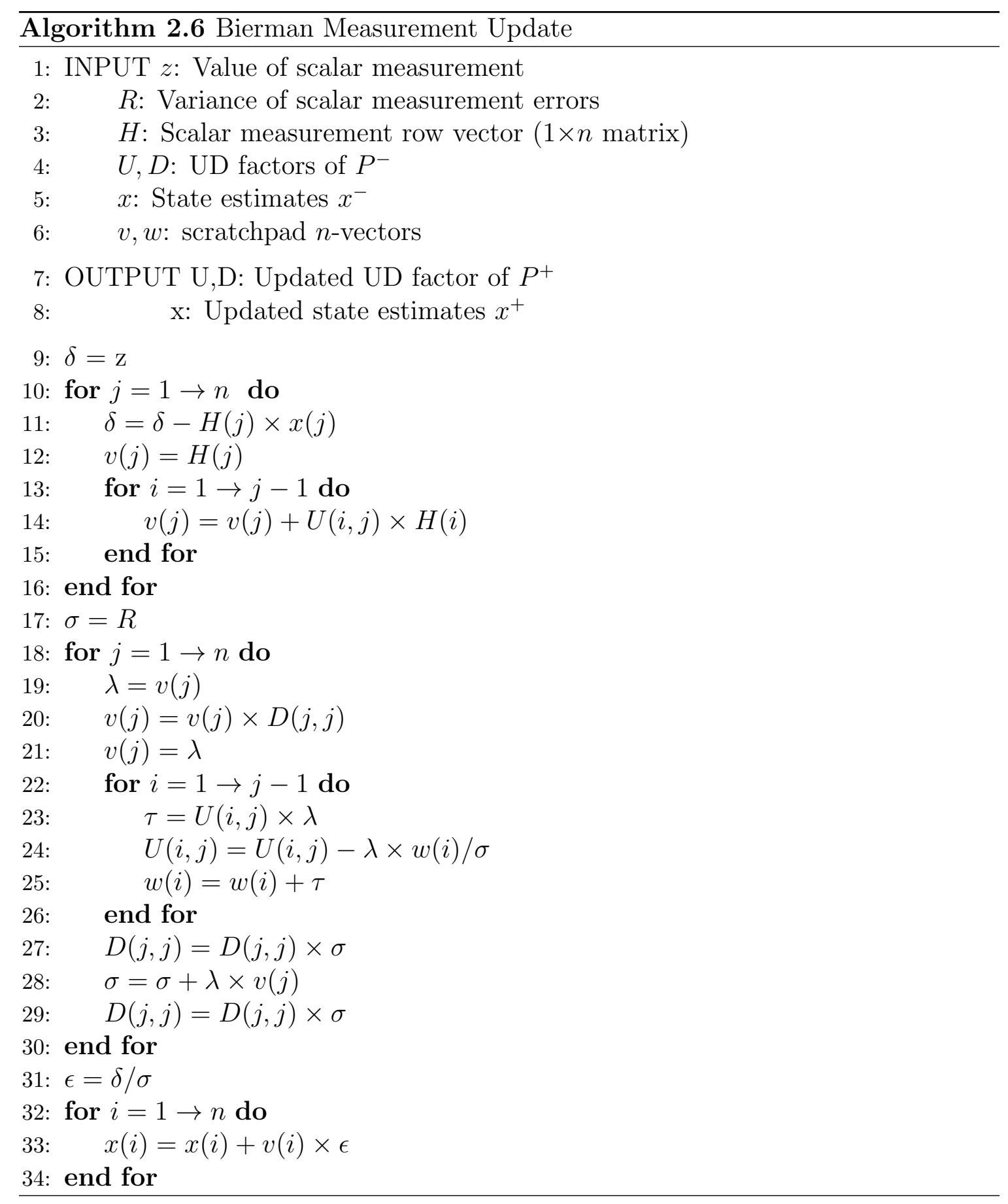

information found in the following sections with respect to the UKF is a summary of $[5,8,34,39,40]$. 


\subsubsection{The Unscented Transformation}

The unscented transformation (UT) is an approximate method to propagate the mean and covariance through non-linear transformations. In the UT, a set of weighted samples (sigma points) $S_{i}=\left\{\mathcal{X}_{i}, W_{i}\right\}$ are carefully chosen such that their statistic properties (mean and covariance) represent the prior distribution of the state variables. Each sigma point is propagated through the nonlinear system functions and a set of transformed sigma points are generated. The transformed set of sigma points and their weights are used to calculate the means and covariances of the posterior probability distribution of the state variables. The UT is similar to a Monte-Carlo method (MC) on the surface. However, there is a big difference between them. Unlike the MC, no random sampling is performed in the UT. Instead, a relatively small number of the sample points are chosen deterministically in the UT.

Let us start with an example of the UT. Suppose that we have the $n$-dimensional random variable $x$ with the mean $\bar{x}$ and the covariance $P_{x x}$. We have another random variable $y$. The random variables $x$ and $y$ are related through a non-linear function,

$$
y=f(x)
$$

The objective of the unscented transformation is to yield the mean $\bar{y}$ and covariance $P_{y y}$ of the variable $y$ by using a set of sigma points.

In order to approximate the distribution of an $n$-dimensional random variable $x, 2 n+1$ weighted points are created by Eqn.(2.78). The sigma points are chosen such that the weighted "sample mean and covariance" of the the sigma points match 
$\left(\bar{x}, P_{x x}\right)$.

$$
\begin{array}{lrl}
\mathcal{X}_{0}=\bar{x} & W_{0}=l /(n+l) \\
\mathcal{X}_{i}=\bar{x}+S_{i} & W_{i}=1 / 2(n+l) \\
\mathcal{X}_{i+n}=\bar{x}-S_{i} & W_{i+n}=1 / 2(n+l)
\end{array}
$$

where $i=1, \ldots, n$ and $n$ denotes the dimension of random variable $x$. The distribution of the random variable $x$ is characterized by its mean $\bar{x}$ and covariance $P_{x x}$. The term $l$ is a fine tuner that can be used to reduce the overall prediction error. The term $S_{i}$ is the $i$ th column of the matrix square root of $(n+l) P_{x x}$. The term $S_{i}$ can be obtained by Cholesky factorization. (Cholesky Factorization is presented in Algorithm 2.1)

$$
\begin{aligned}
A & =S_{i} S_{i}^{T}=(n+l) P_{x x} \\
S_{i} & =\operatorname{cholesky}(A)=\sqrt{(n+l) P_{x x}}
\end{aligned}
$$

where $\sqrt{(n+l) P_{x x}}$ is a upper triangular matrix. The matrix $S$ is generated through Cholesky factorization. The $i$ th sigma point has its corresponding normalized weight $W_{i}$. The weights of sigma points are normalized such that $\sum_{i=1}^{2 n+1} W_{i}=1$. The following procedures are performed to obtain the mean and covariance of $y$ :

1. The non-linear function $f(\cdot)$ is applied to each sigma point to compute its corresponding transformed sigma point:

$$
\mathcal{Y}_{i}=f\left(\mathcal{X}_{i}\right) \quad i=1, \ldots, 2 n+1
$$

2. The mean of $y$ is then computed by the transformed sigma points and their 
respective weights:

$$
\bar{y}=\sum_{i=1}^{2 n+1} W_{i} \mathcal{Y}_{i}
$$

3. The covariance of $y$ is finally computed by the weights and the product of transformed points,

$$
P_{y y}=\sum_{i=1}^{2 n+1} W_{i}\left[\mathcal{Y}_{i}-\bar{y}\right]\left[\mathcal{Y}_{i}-\bar{y}\right]^{T}
$$

The state estimate of the UT represented by the mean and covariance has the accuracy of the second order of the Taylor series expansion of $f(x)$ for any non-linear function [5]. The implementation of the UT is more straightforward since it is not necessary to derive the Jacobians which is required in EKF. The parameter $l$ is a fine tuner to scale the error arising from the higher order terms. Since all higher order terms are simply truncated in the EKF implementations, the UT is an improvement over the EKF and yields more accurate state estimation theoretically.

\subsubsection{Implementation of the Unscented Kalman Filter}

In order to put forward the UKF algorithm, the unscented transformation needs to be incorporated in the Kalman filtering procedures as follows:

1. Propagate the state variable $\hat{x}(k+1 \mid k)$ and its corresponding covariance $P(k+$ $1 \mid k)$. The state variable $x$ is concatenated with the process noise $w_{k}$ which is a $q$-item vector. The augmentation is shown in Eqn. (2.82)

2. Predict the expected measurement $\hat{z}(k+1 \mid k)$ and its corresponding covariance $P_{w w}(k+1 \mid k)$.

3. Update the cross-correlation matrix $P_{x z}(k+1 \mid k)$.

Before we apply the UKF described in [6], the $n$-dimension state vector $x$ needs to be redefined by concatenating the $q$-term process noise. The dimension of the augmented 
state vector is $n^{a}=n+q$. (For example, $n=3, q=3$ and $n^{a}=6$.)

$$
x_{k}^{a}=\left[x_{k}^{T}, w_{k}^{T}\right]^{T}
$$

The term $w_{k}$ denotes the process noise which is a $q$-dimensional vector. The process model can be rewritten as a function of $x_{k}^{a}$,

$$
x_{k+1}=f\left(x_{k}^{a}, u(k)\right)
$$

The augmented state estimate $\hat{x}_{k}^{a}$ and its covariance matrix $P_{k}^{a}$ are used by the unscented transformation to generate $2 n^{a}+1$ sigma points:

$$
\hat{x}_{k}^{a}=\left[\begin{array}{c}
\hat{x}_{k} \\
0_{q \times 1}
\end{array}\right] \quad P_{k}^{a}=\left[\begin{array}{cc}
\hat{P}_{k} & 0 \\
0 & Q_{k}
\end{array}\right]
$$

where $Q_{k}$ is the covariance matrix of the process noise.

The correlated process noise can be easily incorporated into the augmented state vector $x^{a}$ and covariance matrix $P^{a}$. The augmented matrices contain the covariance on the diagonal and the correlations of the state errors and the process noises on the off-diagonal elements. The augmentation method requires additional sigma points which increase the computational cost. However, the process noise can be processed with the same order of accuracy as the state vector. If the measurement model is nonlinear and the elements of the measurement noise are correlated with each other or with the process noise, then the augmented vector needs to include the measurement terms.

The UKF implementation is given as follows:

1. The augmented state vector $x_{k}^{a}$ with the mean $\bar{x}^{a}$ and covariance $P_{k}^{a}$ can be 
approximated by $2 n+1$ weighted points given by

$$
\left[\mathcal{X}_{k}, W_{k}\right]=\operatorname{sigmaPoints}\left(x_{k}^{a}, P_{k}^{a}\right)
$$

where sigmaPoints is a function that is implemented by using Eqn (2.78) in Sec. 2.6.1. This function is to create the sigma points which will be propagated through the system model. The outputs of the function are $W_{k}$ and $\mathcal{X}_{k}$. The term $W_{k} \in \Re^{2 n^{a}+1}$ is a vector of weights and $\mathcal{X}_{k}=\left\{x_{i, k}\right\}_{\left\{i=1, \ldots, 2 n^{a}+1\right\}}$ denotes $2 n^{a}+1$ sigma points. The $2 n^{a}+1$ sigma points and the corresponding weights are generated to approximate the augmented state space $x_{k}^{a}$ and the covariance $P_{k}^{a}$.

2. Each sigma point is propagated through the system model $f(\cdot)$ to compute its corresponding transformed sigma point:

$$
\mathcal{X}_{i, k}=f\left(\mathcal{X}_{i, k-1}, u_{k-1}\right)
$$

where $j=1, \ldots, 2 n^{a}+1$

3. The a priori estimate is then computed by weighted mean of the transformed sigma points and their corresponding weights:

$$
\hat{x}_{k}^{-}=\sum_{i=1}^{2 n^{a}+1} W_{i, k}\left(\mathcal{X}_{k}\right)_{i}
$$

4. The last step of propagation stage is to compute the priori error covariance as:

$$
P_{k}^{-}=\sum_{i=1}^{2 n^{a}+1} W_{i, k}\left[\hat{\mathcal{X}}_{i, k}^{-}-\hat{x}_{k}^{-}\right]\left[\hat{\mathcal{X}}_{i, k}^{-}-\hat{x}_{k}^{-}\right]^{T}
$$


5. In order to implement the update stage, we must compute the predicted observation of each sigma point $\mathcal{X}_{i, k}$ through the measurement function:

$$
z_{i, k}=h\left(\mathcal{X}_{i, k}\right), i=1, \cdots, 2 n^{a}+1
$$

6. Then the a priori expected measurement vector is computed by the weighted mean of the predicted observation for each sigma point:

$$
\hat{z}_{k}^{-}=\sum_{i=1}^{2 n^{a}+1} W_{i, k} z_{i, k}
$$

7. Compute the error covariance matrices:

$$
\begin{aligned}
P_{\hat{z}_{k} \hat{z}_{k}} & =\sum_{i=1}^{2 n^{a}+1} W_{i, k}\left(\hat{z}_{i, k}-\hat{z}_{k}^{-}\right)\left(\hat{z}_{i, k}-\hat{z}_{k}^{-}\right)^{T}+R \\
P_{\hat{x}_{k} \hat{z}_{k}} & =\sum_{i=1}^{2 n^{a}+1} W_{i, k}\left(\hat{\mathcal{X}}_{i, k}-\hat{x}_{k}^{-}\right)\left(\hat{z}_{i, k}-\hat{z}_{k}^{-}\right)^{T}
\end{aligned}
$$

where:

- The term $P_{\hat{z}_{k} \hat{z}_{k}}$ is the innovation covariance and $P_{\hat{x}_{k} \hat{z}_{k}}$ specifies the cross correlation matrix.

- The term $\mathrm{R}$ is the covariance matrix of the measurement noise;

- The term $\hat{z}_{i, k}$ specifies the predicted measurement for each sigma point and $\hat{z}_{k}^{-}$represents the priori expected measurement.

- The term $\hat{\mathcal{X}}_{i, k}$ denotes each transformed sigma point and $\hat{x}_{k}^{-}$specifies the priori state estimate.

8. Compute the Kalman gain:

$$
K_{k}=P_{x z} P_{z z}^{-1}
$$


9. Using the priori measurement vector $\hat{z}_{k}^{-}$to compute the a posteriori state estimate:

$$
\hat{x}_{k}=\hat{x}_{k}^{-}+K_{k}\left(z_{k}-\hat{z}_{k}^{-}\right)
$$

10. The last step of the update stage is to compute the a posteriori estimate of the error covariance as following:

$$
P_{k}=P_{k}^{-}-K_{k} P_{\hat{z}_{k} \hat{z}_{k}} K_{k}^{T}
$$

11. Go to the step 1 for the next time step and create a new set of sigma points with $P_{k}$ and $\hat{x}_{k}$.

The UKF is much easier to implement than the EKF since no Jacobian or Hessians are calculated at any point of the algorithm. This is a major advantage of the UKF implementation over the EKF implementation. According to [8], the computational cost of the UKF is of the same order as the computational cost of the EKF. Superior performance of the UKF over the EKF has been reported in various publications including $[5,8,34,39,40]$.

An example with a highly non-linear system is presented Appendix A.2. The EKF and the UKF are applied to estimate the state variable with respect to this example. The filters and the non-linear model are implemented in Matlab. Figure A.6 and A.9 show the performance of the state estimation with respect to the EKF and UKF. The performance of the filters is quantitatively compared based on the RMSE measure The results of the comparison are illustrated in Figure A.9. Clearly, It demonstrates that the UKF outperforms the EKF significantly on state estimation of the highly non-linear model. 


\subsection{Particle Filters}

The EKF and UKF can only capture the first and second moments of state distribution. When a system model is highly non-linear or its true posterior probability density function (PDF) of the state distribution is non-Gaussain, both the EKF and the UKF are prone to yield large errors and even diverge [10,27]. The particle filter $(\mathrm{PF})$ has appealing convergence properties without being subject to any linearity or Gaussianity constraints. Particle filtering is a technique to implement a recursive Bayesian filter using Monte Carlo (MC) methods and sequential importance sampling(SIS) [41]. Unlike the UKF which uses a deterministic set of samples to approximate the PDF, the PF uses a point mass to represent probability densities. It approximates the posterior PDF by a set of random samples (particles) $N$, with associated weights. The particle filter is also referred to as the Sequential Monte Carlo method. It uses Bayesian analysis to construct the posterior PDF of the state vector $x_{k}$ given all statistical information available.

\subsubsection{Monte Carlo Approach}

The Monte Carlo(MC) approach is a method to solve Bayesian estimation problems by replacing complex analytic or unknown probability distributions with sample based representations. The MC method is to generate independent random samples from a probability distribution $P(X)$. Suppose that $N$ samples (independent and identically

distributed random variables), $\{X(i)\}_{i=1}^{N}$ are drawn from the density $p(X)$ to produce an estimate of the empirical distribution $\hat{P}(X)$.

$$
\hat{P}(X)=\hat{p}_{n}(X)=\frac{1}{N} \sum_{i=1}^{N} \delta(X-X(i))
$$


where $\delta(\cdot)$ denotes a Dirac delta function. The distribution $\hat{P}(X)$ can be used to approximate a probability density function with sums,

$$
\begin{aligned}
I_{N}(f) & =\frac{1}{N} \sum_{i=1}^{N} f(X) \delta(X-X(i))=\frac{1}{N} \sum_{i=1}^{N} f(X(i)) \\
\underset{N \rightarrow \infty}{a . s .} & I(f)=\int f(X) p(X) d X
\end{aligned}
$$

where $I_{N}(f)$ is unbiased and converges to $I(f)$ when $\mathrm{N}$ is approaching infinity according to the strong Law of Large Numbers.

Example: In order to explain the difficulty to approximate a probability density function by using the Monte Carlo method, let us start from an simple application of a constant estimation problem. Suppose that we have a Gaussian random variable and we would like to estimate its mean and variance by using the Monte Carlo method. Gaussian distribution can be expressed analytically as follows:

$$
x \sim \mathcal{N}\left(m_{x}, \sigma_{x}^{2}\right) \quad \text { and } \quad P(x)=\frac{1}{\sqrt{2 \pi \sigma_{x}^{2}}} \exp \left\{\frac{-\left(x-m_{x}\right)^{2}}{2 \sigma_{x}^{2}}\right\}
$$

where the mean $m_{x}$ and the variance $\sigma_{x}$ can be obtained by the following equations.

$$
\begin{aligned}
m_{x} & =\int x P(x) \mathrm{d} x \\
\sigma_{x}^{2} & =\int\left(x-m_{x}\right)^{2} P(x) \mathrm{d} x
\end{aligned}
$$

In contrast, the Monte Carlo method is to draw $N$ samples from a Gaussian distribution. Assuming perfect sampling, $x_{i} \sim \mathcal{N}\left(m_{x}, \sigma_{x}^{2}\right)$, we have the estimate of Gaussian distribution as

$$
\hat{P}(x) \approx \frac{1}{N} \sum_{i=1}^{N} \delta\left(x-x_{i}\right)
$$


Now the mean can be rewritten with the estimation of the samples:

$$
\hat{m}_{x}=\int x \hat{P}(x) \mathrm{d} x=\int x \frac{1}{N} \sum_{i=1}^{N} \delta\left(x-x_{i}\right) \mathrm{d} x=\frac{1}{N} \sum_{i=1}^{N} x_{i}
$$

and variance

$$
\begin{aligned}
\hat{\sigma}_{x}^{2} & =\int\left(x-m_{x}\right)^{2} \hat{P}(x) \mathrm{d} x=\int\left(x-m_{x}\right)^{2} \frac{1}{N} \sum_{i=1}^{N} \delta\left(x-x_{i}\right)(x) \mathrm{d} x \\
& =\frac{1}{N} \sum_{i=1}^{N}\left(x_{i}-\hat{m}_{x}\right)^{2}
\end{aligned}
$$

A simulation of the Monte Carlo method is performed in Matlab to estimate a Gaussian random variable with $m_{x}=2$ and $\sigma_{x}^{2}=4$. The simulations results are shown in Figure 2.5 and Figure 2.6. Figure 2.5 illustrates the sample realization of $N=1000$ samples with corresponding estimated mean(solid line) and upper and lower 95\%-confidence limits about the mean. The sample mean and variance for this MC simulation are at: $\hat{x}_{x}=2.05$ and $\hat{\sigma}_{x}^{2}=4$.13. The Gaussian distribution is estimated using a histogram with 100-bins and is shown in Figure 2.6. In contrast, Figure 2.7 shows a near perfect $\mathrm{MC}$ simulation using $N=10^{6}$ samples.

The example above indicates that the $\mathrm{MC}$ methods approximate the distributions and their associated statistic properties by using simulated samples. The MC methods are acceptable as long as high accuracy is not required or run-time is not a critical concern. These methods are typically very time consuming. In order to achieve a higher level of accuracy, tens or hundreds of thousands of samples are required as shown in Figure 2.7. Therefore, it is very difficult to approximate an arbitrary distribution by using the Monte Carlo methods directly. An extension of the Monte Carlo method has been developed to approximate a distribution more effectively. 


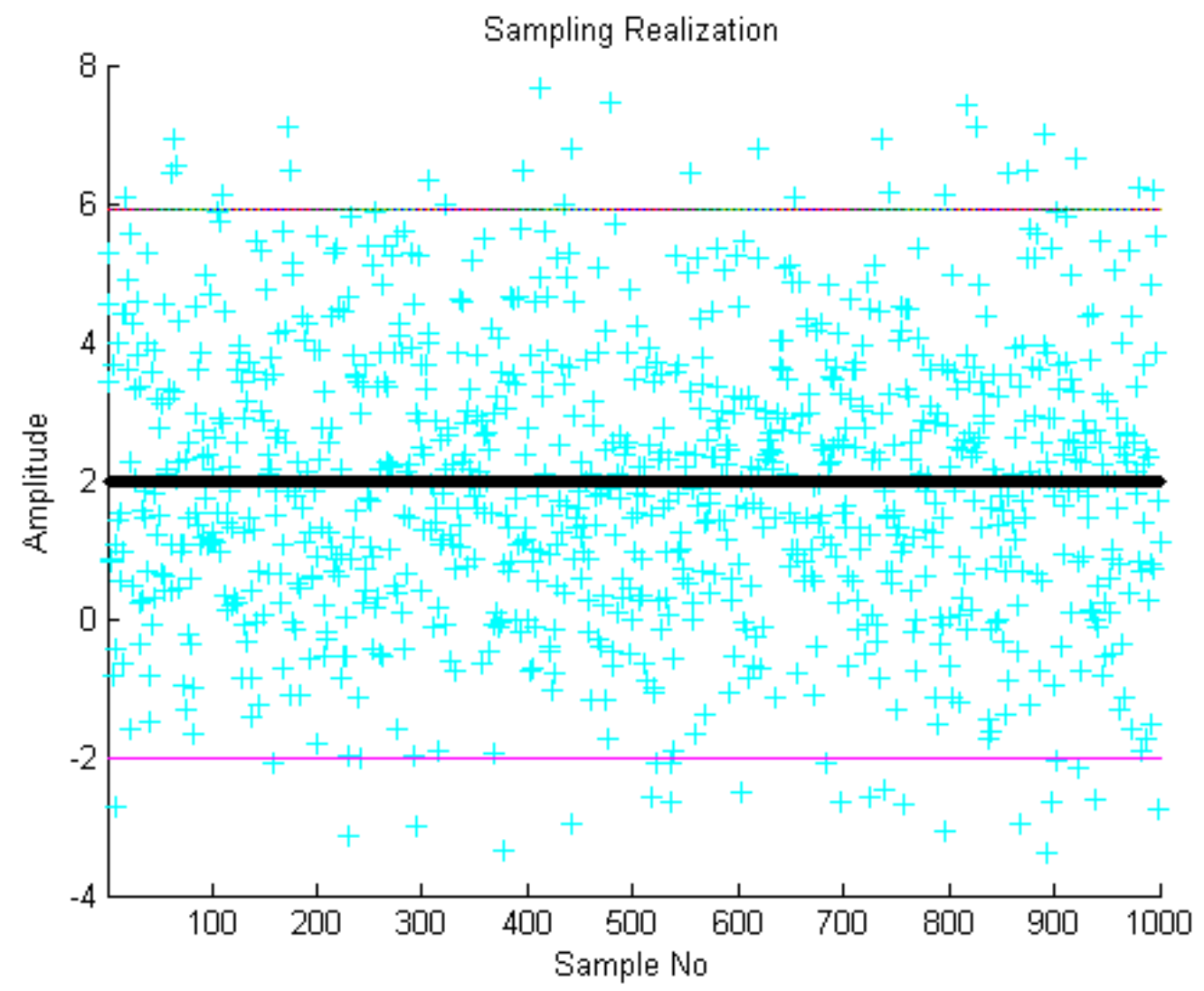

Figure 2.5: $\operatorname{Samples}(N=1000)$ of Monte Carlo Simulation for a Gaussian $\left(m_{x}=2\right.$, $\left.\sigma_{x}^{2}=4\right)$ with $95 \%$ confidence limit.

\subsubsection{Importance Sampling}

One way to mitigate difficulties of sampling directly from a posterior density is based on the concept of importance sampling. The importance sampling method is a fundamental Monte Carlo method. It is the basis of all the underlying particle filtering algorithms developed later on. The importance sampling is a method to compute expectations of one distribution by using random sampling drawn from another distribution. That is, it is a method of simulating samples from a proposal distribution to be used to approximate a posterior distribution by appropriate weighting. Suppose 


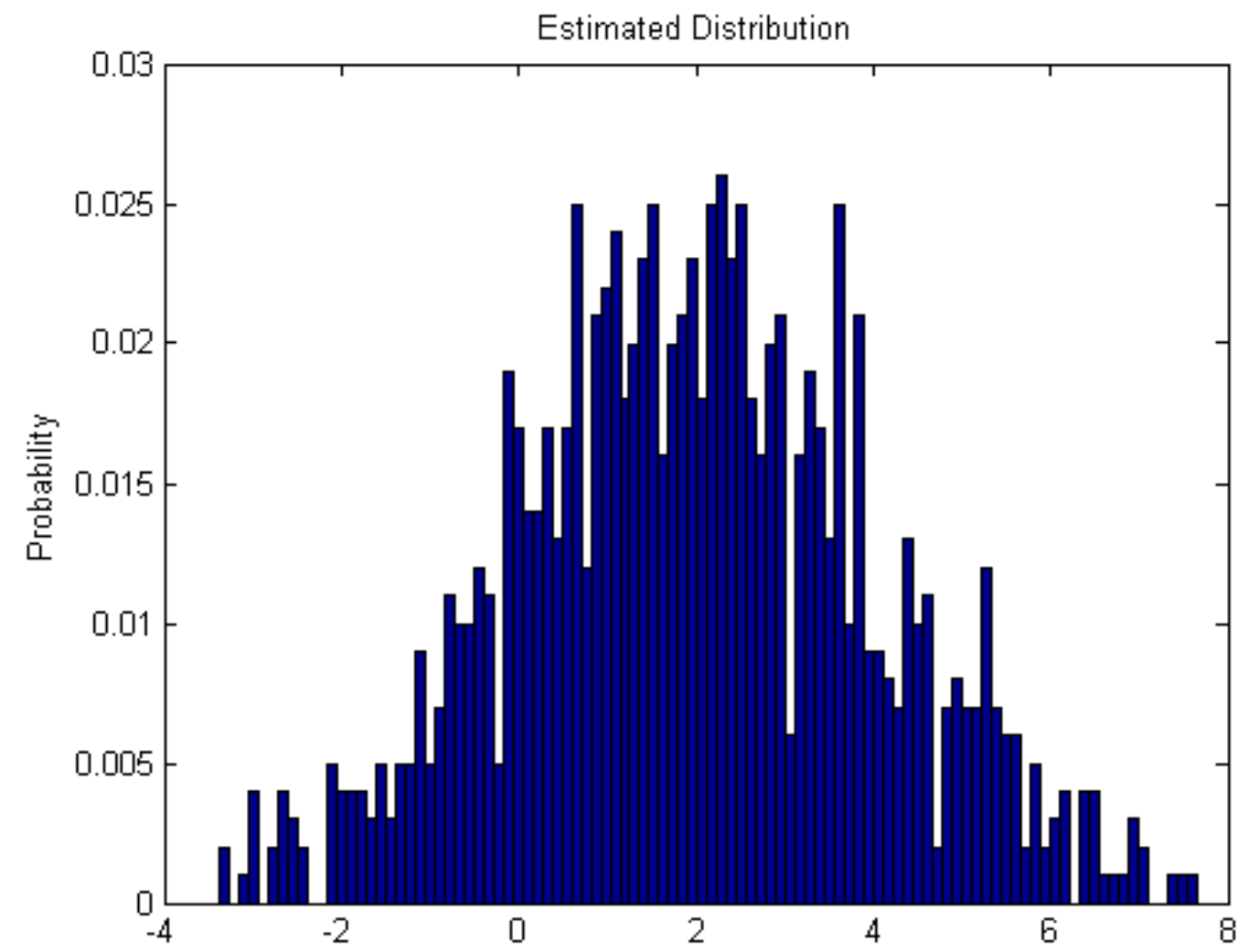

Figure 2.6: Monte Calo Simulation for a $\operatorname{Gaussian}\left(m_{x}=2, \sigma_{x}^{2}=4\right)$ : Estimated Distribution (histogram) from $\operatorname{samples}(N=1000)$

that we want to evaluate a multidimensional integral:

$$
I=\int g(x) d x \quad x \in \mathbb{R}^{n_{x}}
$$

and

$$
g(x)=f(x) \times p(x) \quad \int p(x) d x=1
$$

Importance sampling is a generalization of the Monte Carlo approach as follows:

$$
I=\int f(x) d x=\int\left(\frac{f(x)}{q(x)}\right) \times q(x) d x \quad \text { for } \quad \int q(x) d x=1
$$




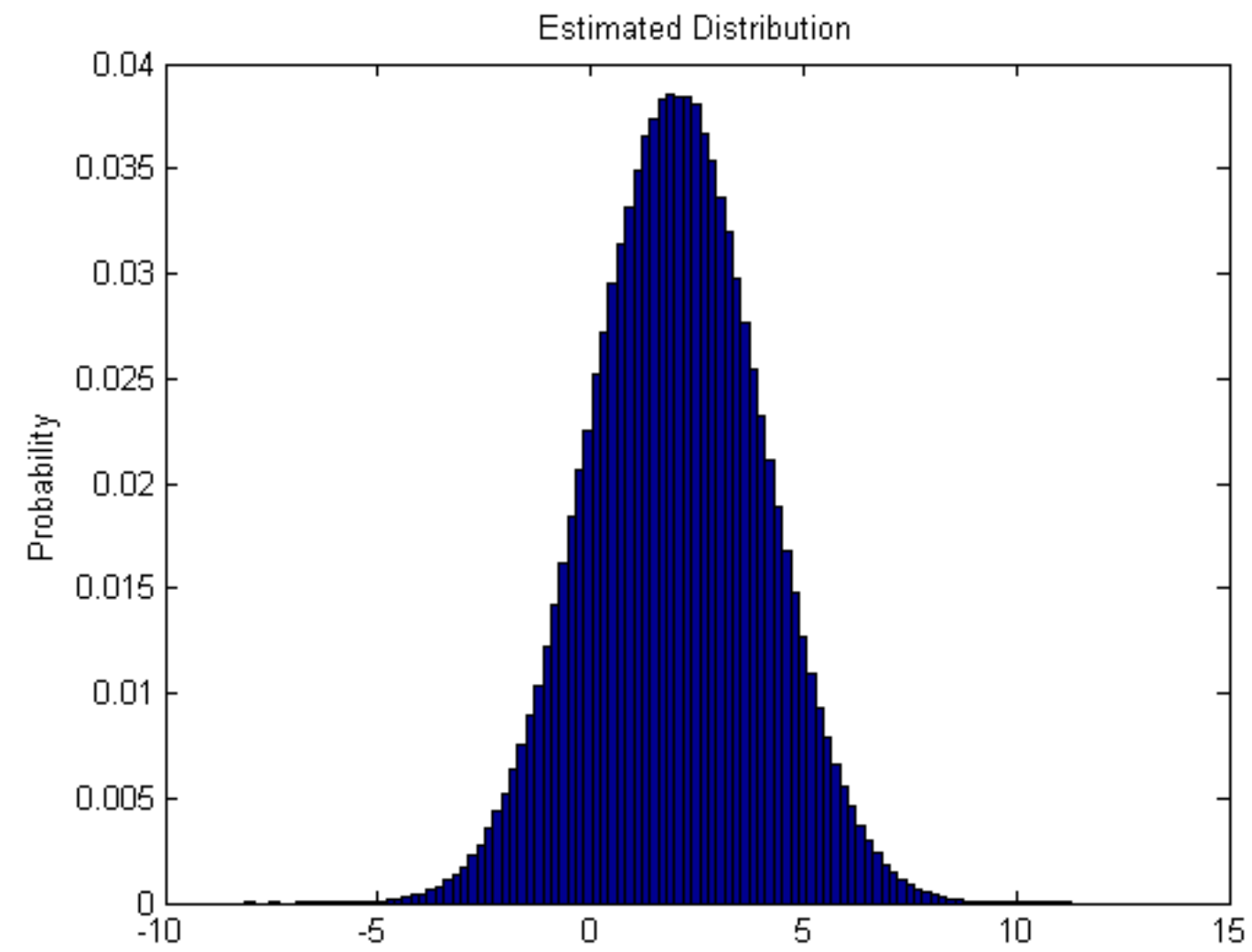

Figure 2.7: Monte Carlo Simulation for a $\operatorname{Gaussian}\left(m_{x}=2, \sigma_{x}^{2}=4\right)$ : Estimated Distribution histogram) of samples $\left(N=10^{6}\right)$

where $q(x)$ denotes the importance sampling distribution. The posterior distribution generally becomes non-uniform after state propagation. That is, $f(x)$ gives more importance to some samples of $f(x)$ than others.

A Monte Carlo estimate of $I$ is computed by using the independent samples $\left\{x_{i} ; i=1, \ldots, N\right\}$. These samples are drawn from $q(x)$ and their weighted sum is expressed as:

$$
I=\frac{1}{N} \sum_{i=1}^{N} f\left(x_{i}\right) \tilde{w}\left(x_{i}\right)
$$

where

$$
\tilde{w}\left(x_{i}\right)=\frac{p\left(x_{i}\right)}{q\left(x_{i}\right)}
$$


are importance weights. If the normalizing factor of the desired density $p(x)$ is unknown, the importance weights needs to be normalized. The estimate $\hat{I}_{N}$ has the following form:

$$
\hat{I}=\frac{\frac{1}{N} \sum_{i=1}^{N} f\left(x_{i}\right) \tilde{w}\left(x_{i}\right)}{\frac{1}{N} \sum_{j=1}^{N} \tilde{w}\left(x_{j}\right)}=\sum_{i=1}^{N} f\left(x_{i}\right) w\left(x_{i}\right)
$$

where the normalized importance weights are given by:

$$
w\left(x_{i}\right)=\frac{\tilde{w}\left(x_{i}\right)}{\sum_{j=1}^{N} \tilde{w}\left(x_{j}\right)}
$$

The importance sampling method is applied in the Bayesian framework where the multi-dimensional probability density $p(x)$ cannot be sampled directly.

\subsubsection{Sequential Importance Sampling}

The aim of the Sequential Monte Carlo methods (SMC) is to estimate the posterior state distribution recursively by using all statistical information available. Suppose that the state estimate of $x_{k}$ is based on the sequence of all available measurements $z_{1: k}=\left\{z_{i}, i=1, \ldots, k\right\}$ up to the time step $k$. The initial density of the state vector is $p\left(x_{0}\right)=p\left(x_{0} \mid z_{0}\right)$. The SMC constructs the posterior PDF $p\left(x_{0: k} \mid z_{1: k}\right)$ up to the time step $k$ given the data $z_{1: k}$. Let $\left\{x_{k}^{i}, w_{k}^{i}\right\}_{i=1}^{N}$ denote a random measure that characterizes the posterior density $p\left(x_{k} \mid z_{k}\right)$, where $\left\{x_{k}^{i}, i=1, \ldots, N\right\}$ is a set of sample points with associated weights $\left\{w_{k}^{i}, i=1, \ldots, N\right\}$. The weights are normalized by Eqn.(2.98) such that $\sum_{i=1}^{N} w_{k}^{i}=1$. The joint posterior density at $k$ can be approximated as follows:

$$
p\left(x_{0: k} \mid z_{1: k}\right) \approx \sum_{i=1}^{N} w_{k}^{i} \delta\left(x_{0: k}-x_{0: k}^{i}\right)
$$

The term $p\left(x_{0: k} \mid z_{1: k}\right)$ is a discrete weighted approximation of the true posterior density. If the samples $x_{0: k}^{i}$ are drawn from an importance density $q\left(x_{0: k} \mid z_{1: k}\right)$, Eqn.(2.96) can 
be rewritten as:

$$
w\left(x_{i}\right) \propto \frac{p\left(x_{0: k}^{i} \mid z_{1: k}\right)}{q\left(x_{0: k}^{i} \mid z_{1: k}\right)}
$$

Suppose that we have samples constituting an approximation to $p\left(x_{0: k-1} \mid z_{1: k-1}\right)$ at the time step $k-1$. When the measurement $z_{k}$ at time step $k$ becomes available, we wish to approximate $p\left(x_{0: k} \mid z_{1: k}\right)$ with a new set of samples. The importance density can be factorized as:

$$
q\left(x_{0: k} \mid z_{1: k}\right)=q\left(x_{k} \mid x_{0: k-1}, z_{1: k}\right) q\left(x_{0: k-1} \mid z_{1: k-1}\right)
$$

In order to derive the weight update equation, the density $p\left(x_{0: k} \mid z_{1: k}\right)$ is rewritten in terms of $p\left(x_{0: k-1} \mid z_{1: k-1}\right), p\left(z_{k} \mid x_{k}\right)$ and $p\left(x_{k} \mid x_{k-1}\right)$ by using the Bayes's rule:

$$
\begin{aligned}
p\left(x_{0: k} \mid z_{1: k}\right) & =\frac{p\left(z_{k} \mid x_{0: k}, z_{1: k-1}\right) p\left(x_{0: k} \mid z_{1: k-1}\right)}{p\left(z_{k} \mid z_{0: k-1}\right)} \\
& =\frac{p\left(z_{k} \mid x_{0: k}, z_{1: k-1}\right) p\left(x_{k} \mid x_{0: k-1}, z_{1: k-1}\right) p\left(x_{0: k-1} \mid z_{1: k-1}\right)}{p\left(z_{k} \mid z_{1: k-1}\right)} \\
& =\frac{p\left(z_{k} \mid x_{k}\right) p\left(x_{k} \mid x_{k-1}\right)}{p\left(z_{k} \mid z_{1: k-1}\right)} p\left(x_{0: k-1} \mid z_{1: k-1}\right) \\
& \propto p\left(z_{k} \mid x_{k}\right) p\left(x_{k} \mid x_{k-1}\right) p\left(x_{0: k-1} \mid z_{1: k-1}\right)
\end{aligned}
$$

The recursive estimate of the importance weights can be derived by substituting Eqn. (2.101) and (2.102) into Eqn. (2.100):

$$
\begin{aligned}
w_{k}^{i} & \propto \frac{p\left(z_{k} \mid x_{k}^{i}\right) p\left(x_{k}^{i} \mid x_{k-1}^{i}\right) p\left(x_{0: k-1}^{i} \mid z_{1: k-1}\right)}{q\left(x_{k}^{i} \mid x_{0: k-1}^{i}, z_{1: k}\right) q\left(x_{0: k-1}^{i} \mid z_{1: k-1}\right)} \\
& =w_{k-1}^{i} \frac{p\left(z_{k} \mid x_{k}^{i}\right) p\left(x_{k}^{i} \mid x_{k-1}^{i}\right)}{q\left(x_{k}^{i} \mid x_{0: k-1}^{i}, z_{1: k}\right)}
\end{aligned}
$$


Furthermore, the importance density $q\left(x_{k}^{i} \mid x_{0: k-1}^{i}, z_{1: k}\right)$ is only dependent on $x_{k-1}$ and $z_{k}$ on the basis of Markov assumptions. The modified weights are then:

$$
w_{k}^{i} \propto w_{k-1}^{i} \frac{p\left(z_{k} \mid x_{k}^{i}\right) p\left(x_{k}^{i} \mid x_{k-1}^{i}\right)}{q\left(x_{k}^{i} \mid x_{k-1}^{i}, z_{k}\right)}
$$

Filtering using the sequential importance sampling (SIS) can recursively propagate the importance weights $w_{k}^{i}$ and the sample points $x_{k}^{i}$ as each measurement is received sequentially. The SIS algorithm is given in Algorithm 2.7.

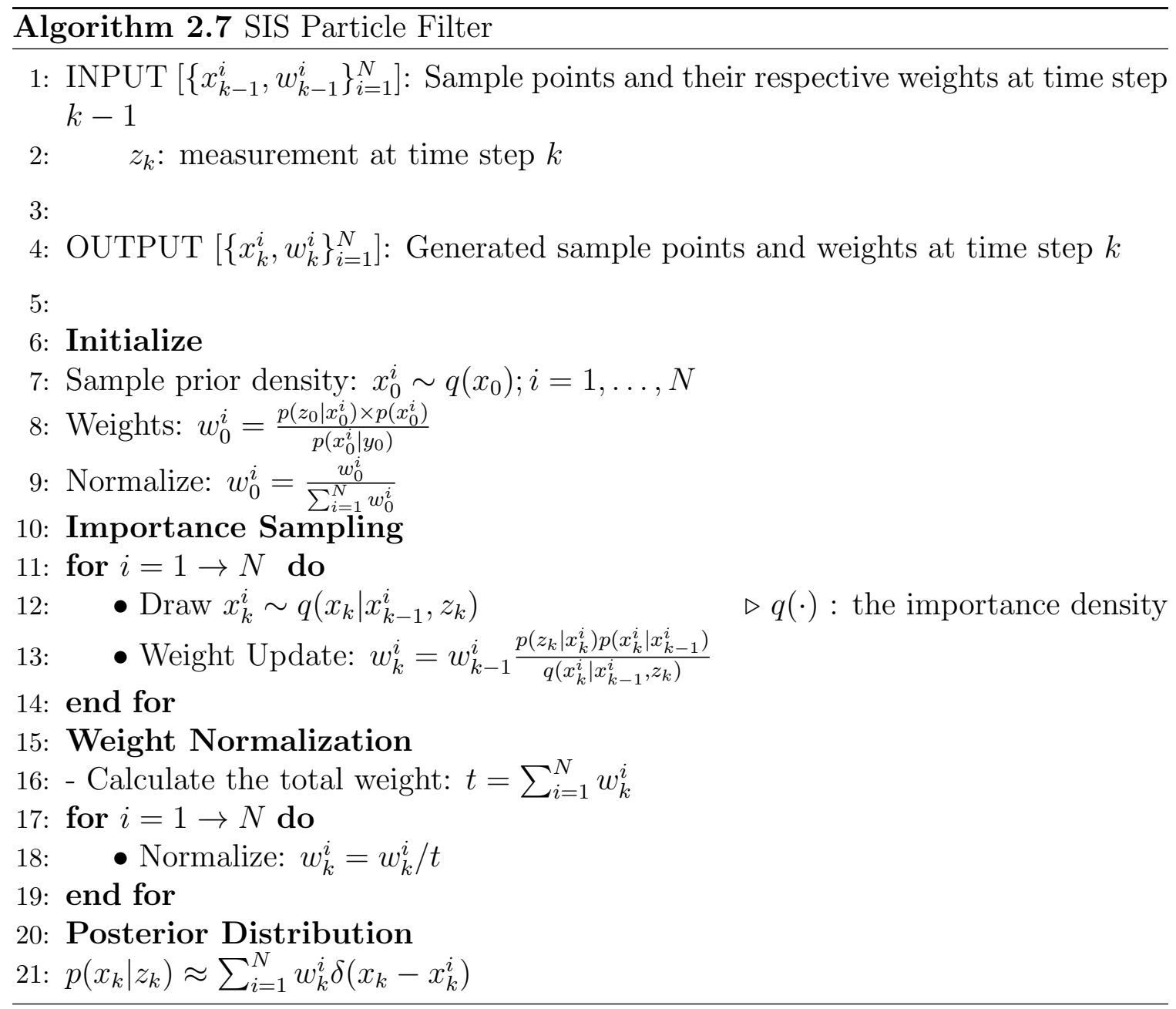

Example: To demonstrate the operations of the SIS particle filter, we present an application to estimate state variable of a simple model. Suppose that we have a 
system dynamics function $f(x)$ and a measurement function as $h(x)$

$$
\begin{aligned}
x_{k} & =f\left(x_{k-1}\right)=\sin \left(x_{k-1}\right)+w_{k} \\
z_{k} & =h\left(x_{k}\right)=5 x_{k}+\eta_{k}
\end{aligned}
$$

where $w_{k}$ represents the process noise and $\eta_{k}$, the measurement noise. They are zero mean, Gaussian Random variables,

$$
w_{k} \sim \mathcal{N}(0,1) \quad \eta_{k} \sim \mathcal{N}(0,2)
$$

and the initial variance of $x_{0}$ is $P\left(x_{0}\right)=1$. The SIS particle filter (Algorithm 2.7) is applied on this example as the following pseudo-code:

\section{- Initialize}

Sample prior density: $x_{0}^{i} \sim q\left(x_{0}\right) ; i=1, \ldots, N$

for $i=1 \rightarrow N$ do

$$
\begin{array}{lr}
x_{0}^{i}=\sin \left(x_{0}\right)+\sqrt{P} \times r v & \triangleright \text { rv: a normal random variable } \mathcal{N}(0,1) \\
w_{k}^{i}=\frac{1}{N} & \triangleright \text { Initialize all samples with the same weights }
\end{array}
$$

\section{end for}

\section{— Update State Estimates Recursively}

$$
\text { for } \begin{aligned}
i & =1 \rightarrow N \text { do } \\
x_{k}^{i} & =\sin \left(x_{k-1}^{i}\right)+w_{k} \\
z_{k}^{i} & =5 x_{k}^{i}+\eta_{k} \\
q_{k}^{i} & =\frac{1}{\sqrt{2 \pi \times \sigma}} \times \exp \left(\frac{-\left(z_{k}-z_{k}^{i}\right)^{2}}{\sqrt{2 \sigma^{2}}}\right)
\end{aligned}
$$$$
\triangleright \operatorname{Draw} x_{k}^{i} \sim q\left(x_{k} \mid x_{k-1}^{i}, z_{k}\right)
$$$$
\triangleright \text { Observation likelihood } \operatorname{PDF} p\left(z_{k} \mid x_{k}^{i}\right)
$$

Note: Weight sampling is based on Gaussian distribution of measurements

$w_{k}^{i}=w_{k-1}^{i} * q_{k}^{i}$

$\triangleright$ Weight Update: $w_{k}^{i}=w_{k-1}^{i} \frac{p\left(z_{k} \mid x_{k}^{i}\right) p\left(x_{k}^{i} \mid x_{k-1}^{i}\right)}{q\left(x_{k}^{i} \mid x_{k-1}^{i}, z_{k}\right)}$

\section{end for}

-Weight Normalization 


$$
t=\sum_{i=1}^{N} w_{k}^{i}-
$$$$
\text { for } i=1 \rightarrow N \text { do }
$$$$
w_{k}^{i}=w_{k}^{i} / t
$$

end for $\triangleright$ Calculate the total weight

$\triangleright$ Normalize

$\triangleright$ Posterior Distribution of $x_{k}$

\section{Degeneracy Problem}

A common problem of the SIS algorithms is the degeneracy of particles' weights. Since the variance of the importance weights increases over time, a few samples hold most of the importance weights, and other samples have zero importance weights in a long run. This causes the algorithm to fail as there are not enough particles that can be used to approximate the posterior distribution. Furthermore, a large portion of computational effort is wasted in propagating the particles with negligibly small weights. Those particles have a very small contribution to the representation of the posterior distribution. A suitable measure of degeneracy is the effective sample size $N_{\text {eff }}$. It is introduced and estimated as follows:

$$
N_{e f f}=\frac{1}{\sum_{i=1}^{N}\left(w_{k}^{i}\right)^{2}}
$$

where a small $N_{\text {eff }}$ indicates a severe degeneracy and vice versa. If the effective sample size is large, say, $N_{e f f}>N / 2$, resampling is generally undesirable since the PDF is well represented.

\subsubsection{Resampling}

Resampling has to be performed in the SIS algorithms whenever a severe degeneracy is observed. Resampling can be thought of as a procedure to eliminate particles with low probability (small weights) and replicate particles with high probability 
(large weights) [42, Chap. 3]. Resampling involves mapping the weighted random measure $\left\{x_{0: k}^{i}, w_{0: k}^{i}\right\}$ onto the equally weighted random measure $\left\{x_{0: k}^{j}, 1 / N\right\}$. So after resampling, a set of $N$ new particles is generated to replace the empirical weighted distribution. All new generated particles have the same weights $w_{k}^{i}=1 / N$.

A direct implementation of resampling is composed of three major steps:

- Generating $N$ independent and identically distributed (i.i.d) variables from the uniform distribution.

- Sorting the generated i.i.d variables in ascending order.

- Comparing the sorted variables with the cumulative sum of normalized weights to decide where to allocate a sample in the uniform distribution.

Systematic Resampling is the most widely used resampling algorithm since it has the least computational complexity. Systematic Resampling is simple to implement and it has minimum computational complexity $O(N)$. Moreover, it minimizes the Monte Carlo variation.

The Systematic Resampling algorithm calculates the cumulative sum of the normalized weight $w_{c}^{i}=\sum_{j=1}^{i} w^{j}$ which is associated to each particle. For example, we have 4 particles and their corresponding weights as $w^{1}=0.1, w^{2}=0.1, w^{3}=0.4$, and $w^{4}=0.4$. The cumulative sum of normalized weight for each particle is $w_{c}^{1}=0.1$, $w_{c}^{2}=0.1+0.1=0.2, w_{c}^{3}=0.1+0.1+0.4=0.6$ and $w_{c}^{4}=0.1+0.1+0.4+0.4=1.0$.

A set of $N$ ordered numbers with regular intervals of $1 / N$ is defined and acts at an equally spaced "comb". The comb is offset by a random number and drawn from a uniform distribution over $\mathcal{U}[1, N]$. The comb is then compared with the cumulative sum of $w_{c}^{i}$ to create a resampled set of numbers $u_{1: N}=\left\{u_{j}: \sum_{k=1}^{i-1} w_{n}^{k} \leq\right.$ $\left.u_{j} \leq \sum_{k=1}^{i} w_{n}^{k}\right\}[10]$. This can be described as a measure to rearrange samples nonuniformly along the uniform distribution on the bases of the different sample weights. 
We can explain this with the simple example of last paragraph. As described in the example, we have $\left[w_{c}^{1}=0.1, w_{c}^{2}=0.2, w_{c}^{3}=0.6\right.$ and $\left.w_{c}^{4}=1.0\right]$. Given that the uniform distribution is divided into $N=4$ parts evenly, we have 3 levels as $u_{1}=0.25$, $u_{2}=0.5$, and $u_{3}=0.75$ respectively. The new sample set is translated from the old sample set as follows

$$
x^{1: 4}=\left\{\begin{array}{lll}
1,2 \rightarrow 1,1 & u_{1}=0.25 & u_{1}>w_{c}^{1} \text { and } u_{1}>w_{c}^{2} \\
\text { None, } & u_{2}=0.50 & w_{c}^{3}>u_{2} \\
3 \rightarrow 3, & u_{3}=0.75 & u_{2}<w_{c}^{3}<u_{3} \\
4 \rightarrow 4, & u_{3}=0.75 & w_{c}^{4}>u_{4}
\end{array}\right.
$$

Eqn.(2.109) shows the sample with label 2 is eliminated and the sample with label 1 is duplicated once. The original set of samples $N=1,2,3,4$ is resampled and translated to a new set of samples which consists of labels of the original set as $1,1,3,4$.

The systematic resampling algorithm is described in Algorithm 2.8.

Example: Figure 2.8 is presented to explain the systematic resampling scheme further with an example of $N=5$ samples. In this example, 5 samples with their corresponding cumulative sum of the normalized weight as $w_{c}^{1: 5}=\{0.2,0.3,0.65,0.7,0.98\}$. 


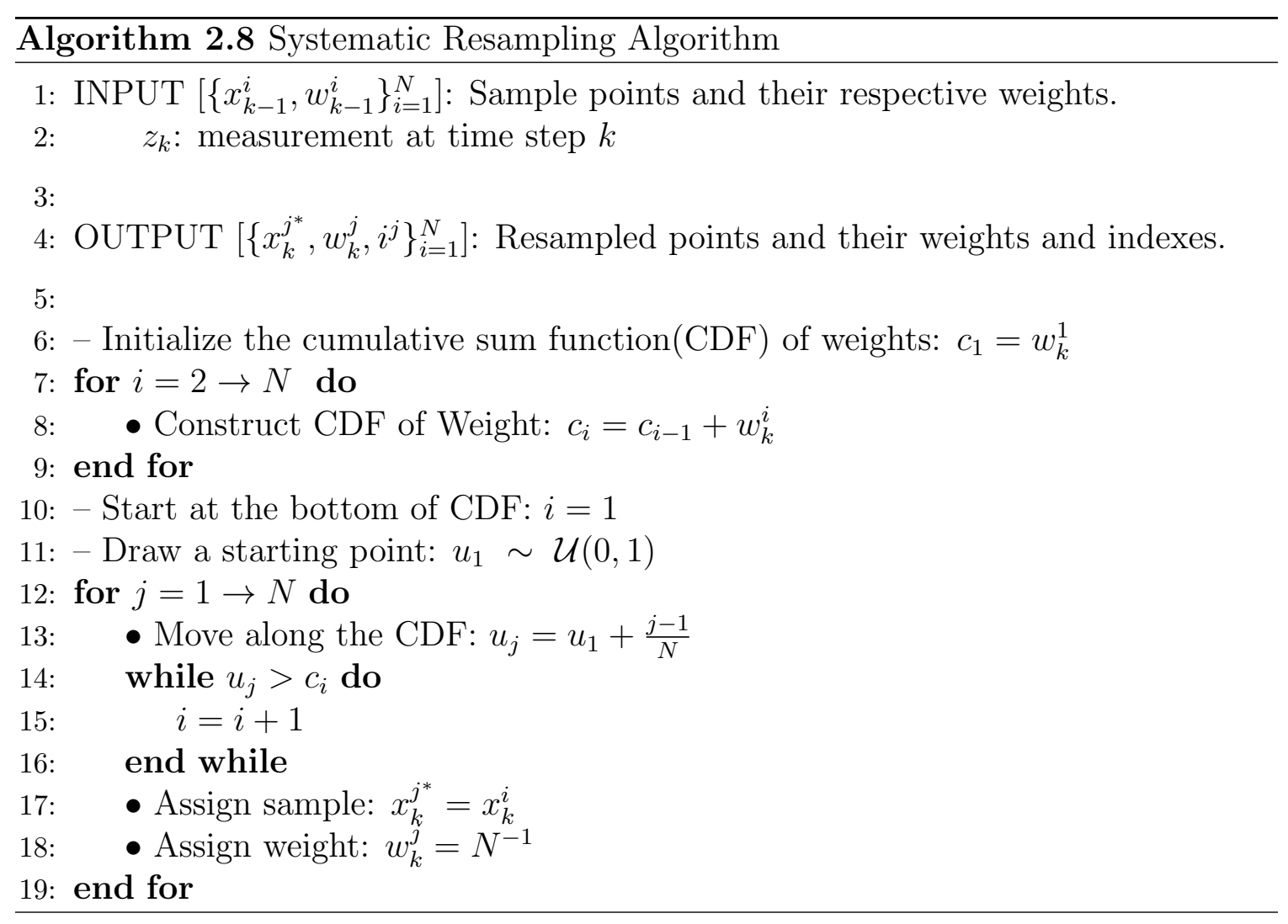

The 5 levels are divided by the lines at $u_{1: 5}=\{0.15,0.35,0.55,0.75,0.95\}$. The resampled set is

$$
x^{1: 5}=\left\{\begin{array}{lll}
\text { None } & u_{1}=0.15 & w_{c}^{1}>u_{2} \\
1,2 \rightarrow 2,2 & u_{2}=0.35 & u_{1}<w_{c}^{1}, w_{c}^{2}<u_{2} \\
\text { None } & u_{3}=0.55 & w_{c}^{3}>u_{3} \\
3,4 \rightarrow 4,4 & u_{3}=0.75 & u_{3}<w_{c}^{3}, w_{c}^{4}<u_{4} \\
5 \rightarrow 5, & u_{3}=0.95 & w_{c}^{5}>u_{5}
\end{array}\right.
$$

The samples with the labels 1,3 in the original set are eliminated and the samples 2, 4 are replicated once for each. Finally, the original set of samples $N=1,2, \ldots, 5$ is translated to the resampled set which consists of labels of the original set as $2,2,4,4,5$. 


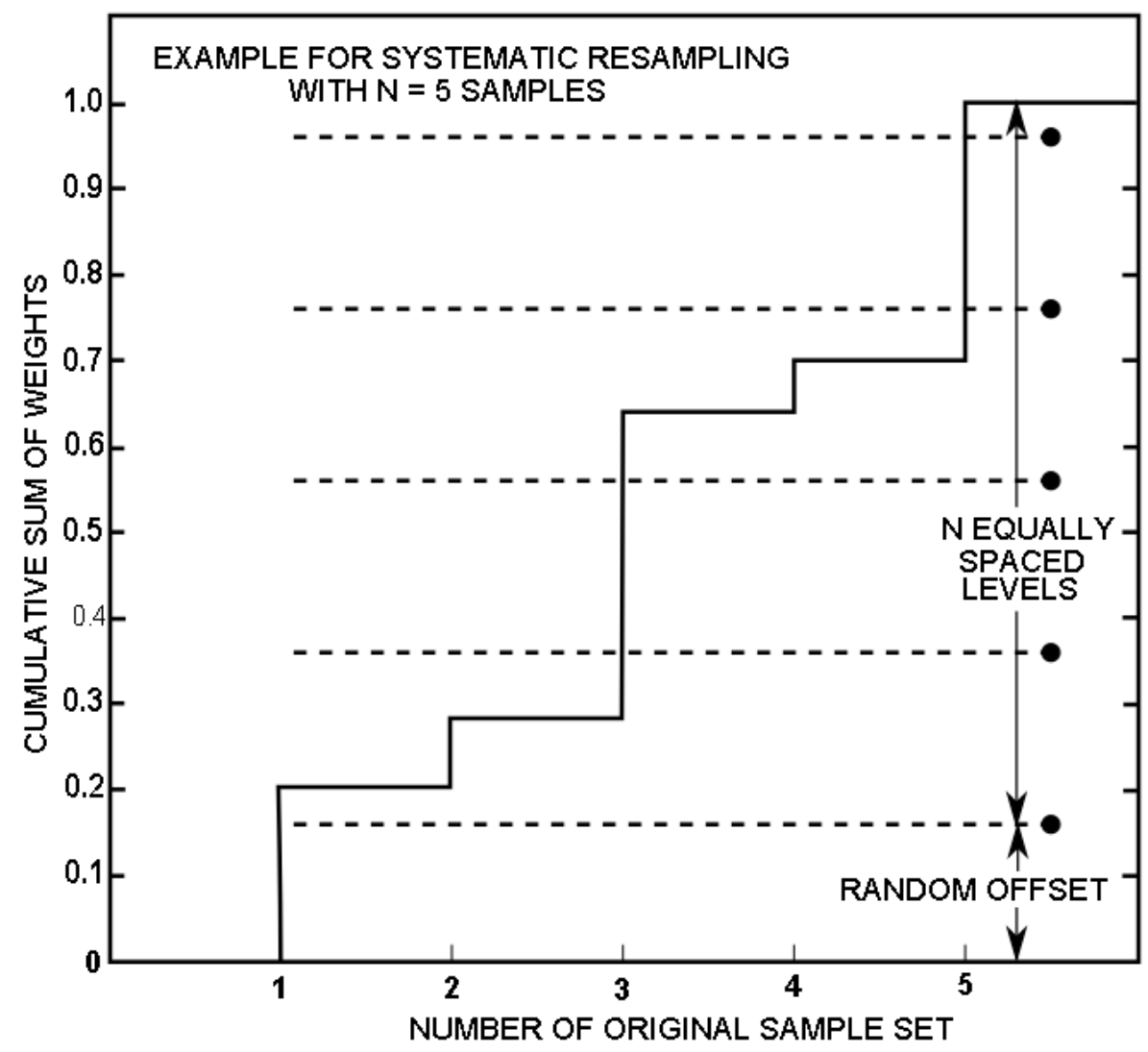

Figure 2.8: Graphical Interpretation of Systematic Resampling: the random measure $\left\{x_{k}^{i}, w_{k}^{i}\right\}_{i=1}^{N}$ is mapped into an equally weighted random measure $\left\{x_{k}^{j^{*}}, w_{k}^{j}\right\}_{j=1}^{N}$. Figure is motivated by [42].

Using the resampling method in conjunction with the SIS particle filter, we can compose what is known as the generic particle filter. It can be used as the basic skeleton of many particle filtering algorithms. The algorithm of the generic particle filter is illustrated in Algorithm 2.9. A flowchart is given in Figure 2.9 to illustrate the flow of the algorithm. 


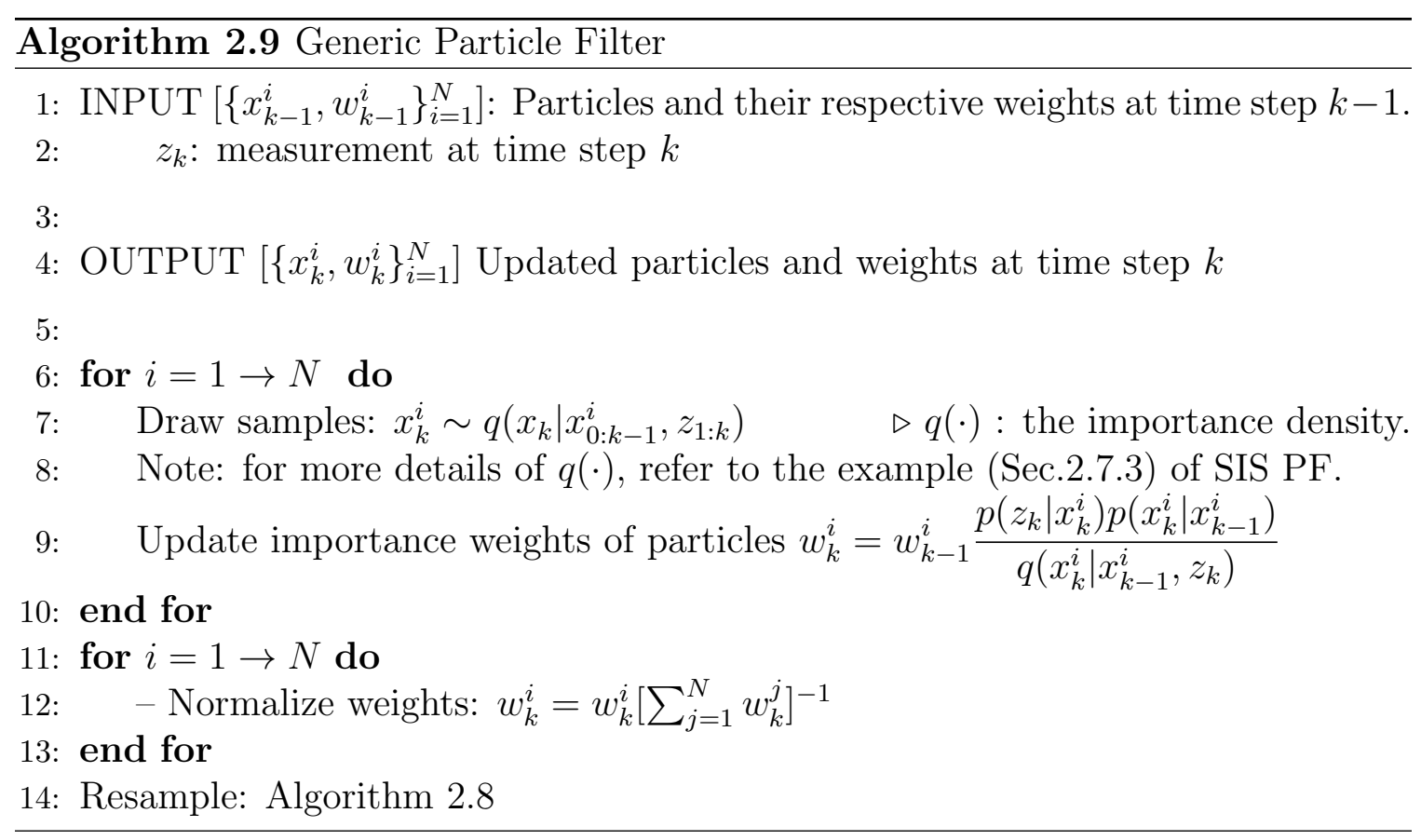

\subsubsection{The Bootstrap Particle Filter}

The sample importance resampling filter was first proposed in 1993 by Gordon, Salmond and Smith [10]. It is the first practical particle filter to be applied to tracking problems. This filter is also referred to as the bootstrap PF. The bootstrap filter and its variants are the most popular algorithms among all particle filter techniques due to its simplicity of implementation. It is one of the non-linear filters used for comparative purposes later in this thesis.

The bootstrap filter is derived from the generic particle filter. It simply uses the prior distribution to calculate the importance sampling distribution.

$$
q\left(x_{k} \mid x_{k-1}, z_{k}\right)=p\left(x_{k} \mid x_{k-1}\right)
$$


The weight calculation is simplified as shown in following equations.

$$
\begin{aligned}
w_{k} & =w_{k-1} \times \frac{p\left(z_{k} \mid x_{k}\right) p\left(x_{k} \mid x_{k-1}\right)}{p\left(x_{k} \mid x_{k-1}, z_{1: k}\right)} \\
& =w_{k-1} \times \frac{p\left(z_{k} \mid x_{k}\right) p\left(x_{k} \mid x_{k-1}\right)}{p\left(x_{k} \mid x_{k-1}\right)} \\
& =w_{k-1} \times p\left(z_{k} \mid x_{k}\right)
\end{aligned}
$$

The implementation of the bootstrap filter requires resampling at every time step. This can save time of calculating a threshold value for resampling. Moreover, since all the weights are set to the same value at each step time, the weight calculation can be simplified further as:

$$
w_{k}=\frac{1}{N}
$$

However, the simplicity of the implementation of the bootstrap filter is also its major shortcoming. Since the importance density is chosen to be independent of the current measurement, the state space is explored without all the available information. This causes the particles to degenerate more quickly at each time step. Moreover, the filter may be sensitive to outliers [41, Chap. 7].

Pseudo-code for the bootstrap filtering algorithm is given in Algorithm 2.10.

\subsection{Chapter Summary}

This chapter started by introducing the history of filtering theory and moved forward to system modelling and Recursive Least-Squares estimation. The theories and implementation methods of the Kalman filter and the EKF were both discussed in this chapter. The parameter tuning methods of the Kalman filter were presented with a simple application. The UKF which is known as an alternative of the EKF, was 
described in detail. This chapter also made use of a few examples to explain the nature and performance of the UKF and the EKF. Finally, this chapter presented the theory of particle filters. It introduced several basic theories of particle filtering including the importance sampling, the sequential importance sampling, the problem of degeneracy, the resampling and the bootstrap particle filtering approach. Several examples were provided to explain the theories and the operations of the algorithms. 


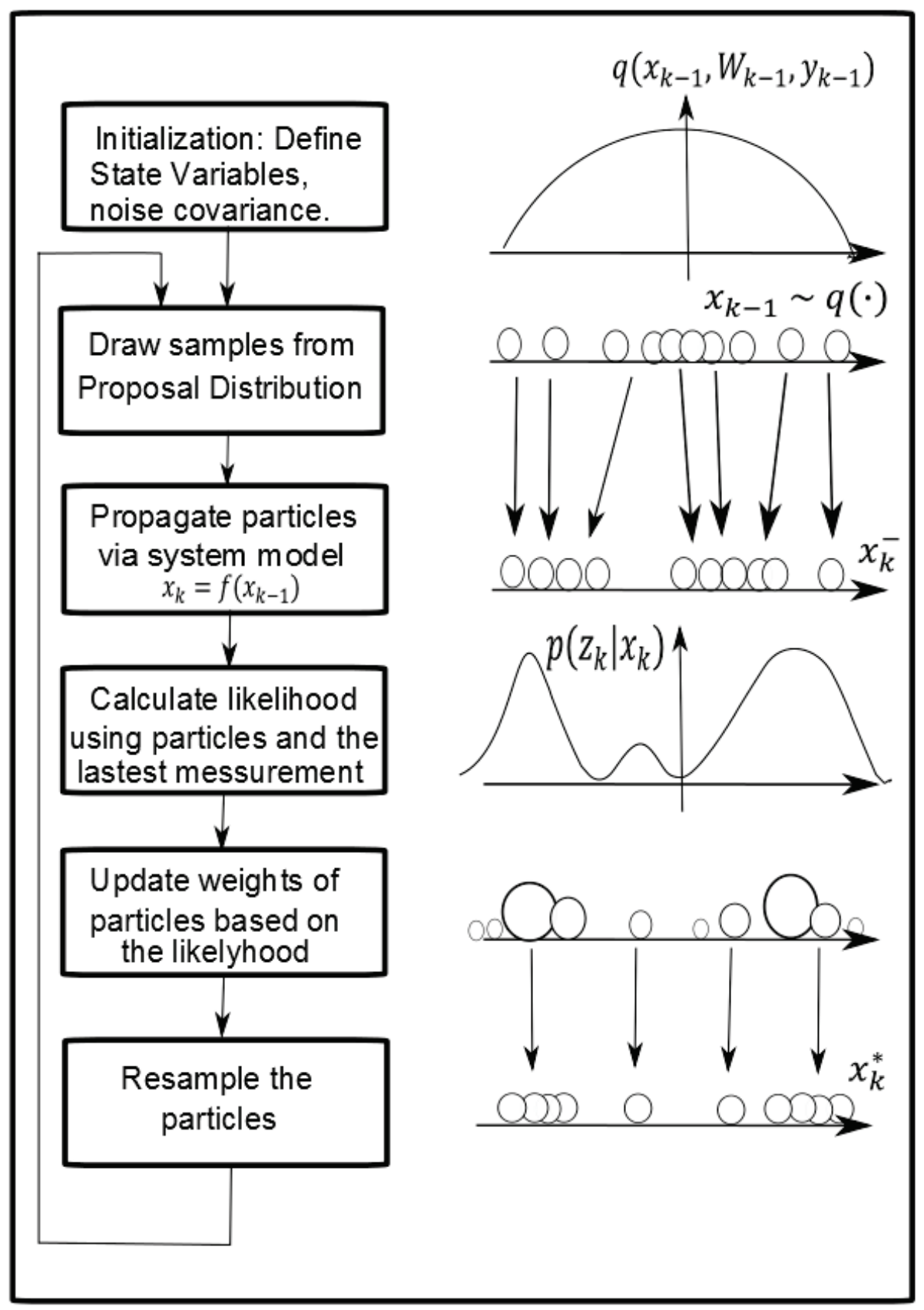

Figure 2.9: Generic Particle Filter Flowchart: particles are sampled, propagated. The weights are update according to the latest measurement. Finally particles are resampled before the process is repeated into the next time step. The particles are denoted by the circles. The radius of the circle corresponds to the particle's weight. 
Algorithm 2.10 The Bootstrap Particle Filter

INPUT: $\left[\left\{x_{k-1}^{i}, w_{k-1}^{i}\right\}_{i=1}^{N}\right]$ : Particles and their respective weights at time step $k-1$. $\quad z_{k}$ : measurement at time step $k$

OUTPUT: $\left[\left\{x_{k}^{j^{*}}, w_{k}^{j}\right\}_{i=1}^{N}\right]$ Updated particles and weights at time step $k$

\section{Initialization:}

- Initialize the process noise covariance matrix as $Q$ and measurement noise covariance matrix as $R$.

5: - Initialize the a priori estimate error covariance matrix as $P_{x}^{-}$.

- Draw a set of initial samples from $x_{0}^{i} \sim P\left(x_{0}\right)(i=1, \ldots, N)$ :

$$
x_{0}^{i}=x_{0}+\sqrt{\left(P_{x_{0}}^{-}\right)} * \mu, \quad \mu \sim \mathcal{N}(0,1)
$$

\section{Importance Sampling and Weight Update For Each Particle:} for $i=1 \rightarrow N$ do

-Draw a set of new particles $\hat{x}_{k}^{i(-)}$ to approximate the a priori importance density $q\left(x_{k} \mid x_{k-1}^{i}\right)$ as:

$$
x_{k}^{i(-)}=f\left(x_{k-1}\right)+\sqrt{Q} \times \mu \times T
$$

where $\mu \sim \mathcal{N}(0,1) ; T$ is the sample time period and $i=1, \ldots, N$. Note: we use the prior distribution $x_{k}^{i(-)}$ to calculate the importance sampling distribution in the bootstrap filter, thus we can set: $x_{k}^{i}=x_{k}^{i(-)}$.

10: $\quad$ Generate the likelihood: $\mathcal{L}_{k}^{i}=P\left(z_{k} \mid x_{k}^{i}\right)$ using the particle $x_{k}^{i}$ and the latest measurement $z_{k}$ as follows:

- Calculate $z_{k}^{i}$ through the measurement model $h(\cdot)$ with the newly generated particles $x_{k}^{i}$ :

$$
z_{k}^{i}=h\left(x_{k}^{i}\right)
$$

- Compute the likelihood $\mathcal{L}_{k}^{i}$ of the current particle given standard deviation of the measurement noise: $\eta$. $\left(\mathcal{L}_{k}^{i}=p\left(z_{k}^{i} \mid z_{k}, x_{k}^{i}\right)\right)$

- Assign each particle a weight, $w_{k}^{i}: \hat{w}_{k}^{i}=\mathcal{L}_{k}^{i} \times w_{k-1}^{i}, i=1, \ldots, N$. where $w_{k-1}^{i}=1 / N$ after each resampling for the bootstrap filter.

end for

15: for $i=1 \rightarrow N$ do

- Normalize the importance weights: $w_{k}^{i}=w_{k}^{i}\left[\sum_{j=1}^{N} w_{k}^{j}\right]^{-1}$

\section{end for}

- Resample using Algorithm 2.8

- Output:Generate the new set as $\left\{\hat{x}_{k}^{i}, \hat{w}_{k}^{i}\right\}$. The state is eventually updated by means of the particle as:

$$
\hat{x}(k)=\frac{1}{N} \sum_{i=1}^{N} \hat{x}_{k}^{i}
$$




\section{Chapter 3}

\section{Simulation and Results for Pose}

\section{Estimation}

\subsection{Introduction}

In this chapter, the non-linear filters are implemented and applied to estimate the positions and orientations of a mobile robot in a simulated environment. We assume that the robot moves on a $2 \mathrm{D}$ horizontal plane and travels along a non-linear trajectory like a circular arc or a spiral in the simulation. The robot's state variables are corrupted by noise and disturbances. The non-linearity in the system dynamics model makes the tracking problem more difficult. The non-linearity feature of the measurement system also adds complexity to this simulation. The measurement system tracks the robot by using the observations of the ranges and bearings with respect to three landmarks. The measurements are also disturbed by zero mean white noise.

The performance of the non-linear filters on the robot's pose estimation is compared and analysed. This chapter also compares the computational complexity of each filter based on the length of time to perform the pose estimate. 


\subsection{Model Description}

\subsubsection{System Dynamic Model}

One refers to the pose estimation problems as the ability to estimate the position and orientation of a mobile robot from noisy sensor data. The mobile robot in the simulation is a three-wheeled vehicle as shown in Figure 3.1. The model of the mobile robot is depicted in Figure 3.2. The robot's model has been derived assuming no slippage in [43]. The robot is driven by its rear wheels and the front wheel is a steering wheel. As described in [43], the combination of position and orientation is referred to as the pose of a robot. The system state of the pose of the robot is defined as $X_{k}=\left(x_{k}, y_{k}, \theta_{k}\right)^{T}$, where $x_{k}$ and $y_{k}$ specify the coordinates of the point $\mathrm{M}$ in Figure 3.2 in the Cartesian plane. The point $\mathrm{M}$ is mid-distance between rear wheels of the robot. The term $\theta_{k}$ denotes the angle between the robot's longitude and $x$-axis of the Cartesian plane. The term $\delta$ represents the turning angle of the front wheel with respect to the robot's longitudinal direction.

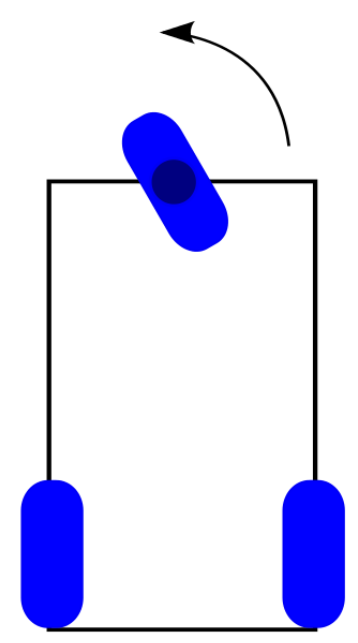

Figure 3.1: Three wheeled omni-steer mobile robot 


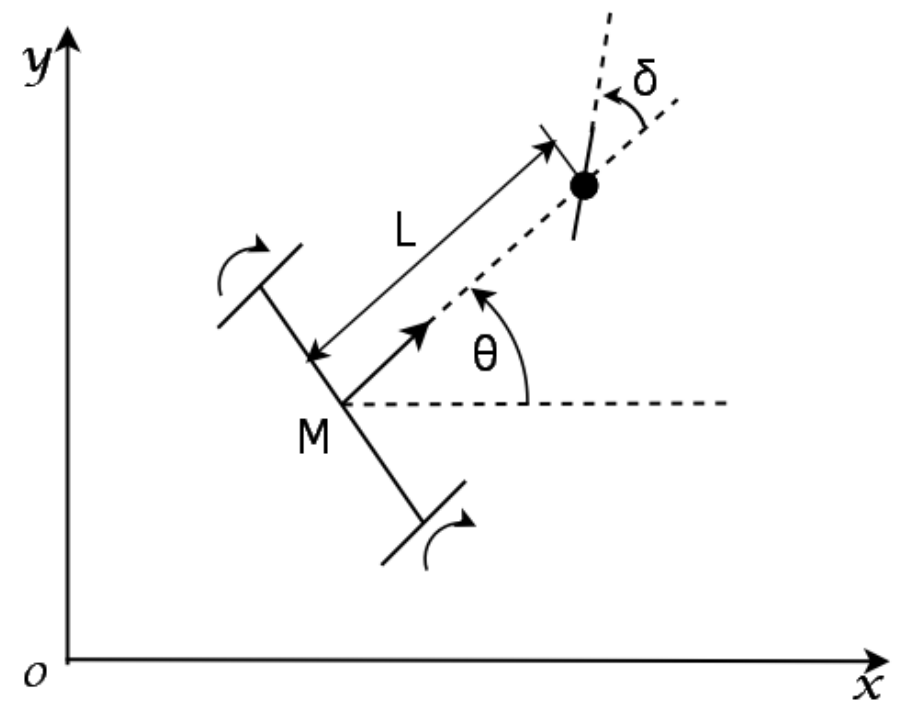

Figure 3.2: Model of Mobile Robot

The robot's model can be described by the following stochastic differential equations (SDE) [44]:

$$
\dot{X}(t)=f(X)+w
$$

where $f(X)$ is a nonlinear system function and $w=\left[w_{x}, w_{y}, w_{\theta}\right]^{T}$ specifies additive random process noise. The differential equation of the state vector of the robot model can be written as

$$
\left[\begin{array}{l}
\dot{x} \\
\dot{y} \\
\dot{\theta}
\end{array}\right]=\left[\begin{array}{c}
v \cos \theta \\
v \sin \theta \\
\frac{v}{L} \tan \delta
\end{array}\right]+\left[\begin{array}{l}
w_{x} \\
w_{y} \\
w_{\theta}
\end{array}\right]
$$

where $v$ specifies the robot longitudinal velocity, and $\dot{\theta}$ is the chassis instantaneous angular velocity. The term $\delta$ characterizes the vehicle's steering wheel angle with respect to the robot longitude, and $\mathrm{L}$ specifies the distance between the rear and front wheels' axles. In all forthcoming simulations, L is set equal to $5 \mathrm{~cm}$.

We assume that the process noise $w$ in Eqn.(3.1) is a zero mean Gaussian random 
variable(GRV). The process noise includes all kinds of errors that can not be detected by odometry such as range error, turn error and drift error. Moreover, the process noise $w$ is independent of the system states. The standard deviations of the process

noise $w$ in all the simulations are $\sigma_{x}=\sigma_{y}=1 \mathrm{~cm}$ and $\sigma_{\theta}=\frac{\pi}{180} \mathrm{rad}$. The covariance matrix of the process noise is defined as: $Q=E\left[w w^{T}\right]$ and $w \sim \mathcal{N}(0, Q)$.

It is not necessary to integrate Eqn.(3.2) analytically to find explicit discrete time equations. However, we perform the state propagation by applying numerical integration (4th order Runge-Kutta method). In order to obtain an accurate state estimate throughout the simulation, we pick a step-size $h=0.0002$ sec to implement $4 t h$ order Runge-Kutta method. All state variables are calculated with absolute error tolerances of $10^{-5} \mathrm{~cm}$ in each sample period $T_{s}=0.2 \mathrm{sec}$.

\subsubsection{Measurement Model}

At each time step, there are three measurements available with respect to three landmarks as shown in Figure 3.3: range $r_{1}, r_{2}$ and bearing $\alpha$. The range $r_{1}$ and $r_{2}$ are the distances from the robot to landmark 1 and landmark 2. Meanwhile, the bearing measurement $\alpha$ represents the angle between the landmark 3 and the mobile robot longitude. 


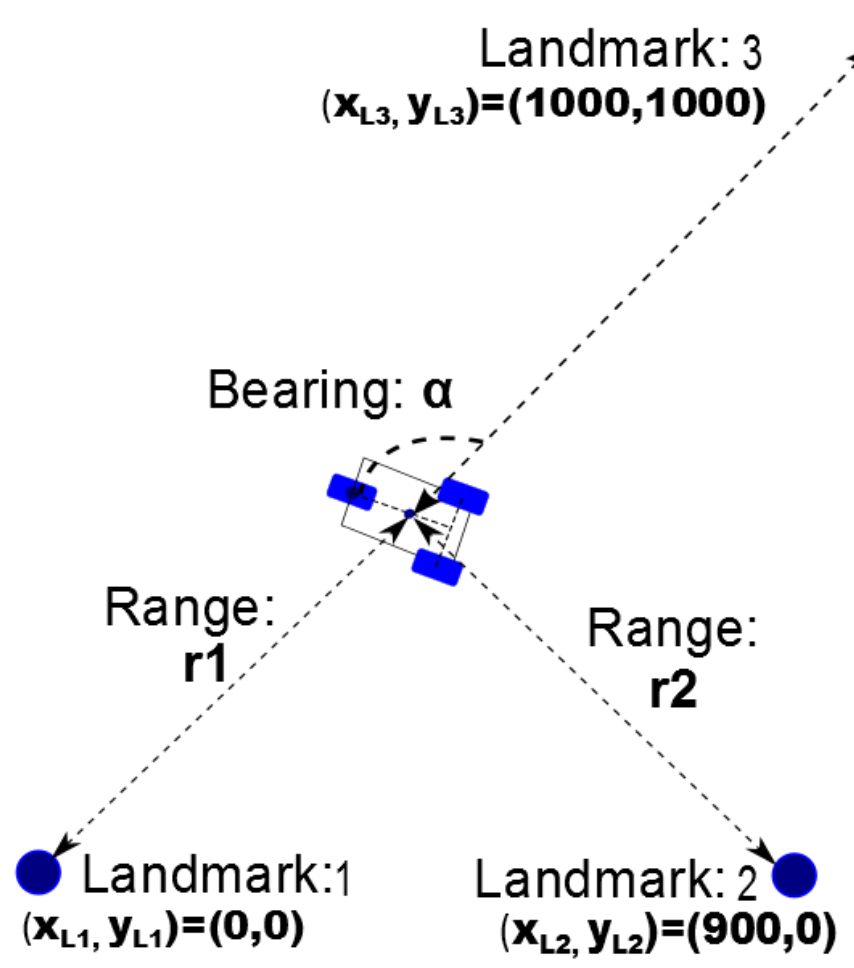

Figure 3.3: Model of Measurement System

The measurement model is defined as

$$
\begin{aligned}
z_{k}=\left[\begin{array}{l}
r 1_{k} \\
r 2_{k} \\
\alpha_{k}
\end{array}\right]=h\left(x_{k}\right)+\eta_{k} \\
=\left[\begin{array}{c}
\sqrt{\left(x_{k}-x_{L 1}\right)^{2}+\left(y_{k}-y_{L 1}\right)^{2}} \\
\sqrt{\left(x_{k}-x_{L 2}\right)^{2}+\left(y_{k}-y_{L 2}\right)^{2}}
\end{array}\right]+\left[\begin{array}{l}
\eta_{r 1} \\
\eta_{r 2} \\
\arctan \left(\frac{y_{L 3}-y_{k}}{x_{L 3}-x_{k}}\right)-\theta_{k}
\end{array}\right]
\end{aligned}
$$


where $\left(x_{L 1}, y_{L 1}\right),\left(x_{L 2}, y_{L 2}\right)$ and $\left(x_{L 3}, y_{L 3}\right)$ denote the Cartesian coordinates of the landmarks 1, 2 and 3 respectively. The terms $x_{k}$ and $y_{k}$ denote Cartesian coordinates of the robot location. The terms $\eta_{r 1}, \eta_{r 2}$ and $\eta_{\alpha}$ represent additive zero-mean Gaussian noise of the measurement, thus $\eta_{r 1} \sim \mathcal{N}\left(0, \sigma_{r 1}^{2}\right), \eta_{r 2} \sim \mathcal{N}\left(0, \sigma_{r 2}^{2}\right)$ and $\eta_{\alpha} \sim \mathcal{N}\left(0, \sigma_{\alpha}^{2}\right)$. The terms $\sigma_{r 1}, \sigma_{r 2}$ and $\sigma_{\alpha}$ are the standard deviations with respect to the range $r 1, r 2$ and the bearing $\alpha$ respectively. Finally, we assume that the standard deviations of the measurement errors are $\sigma_{r} 1=5 \mathrm{~cm}, \sigma_{r} 2=5 \mathrm{~cm}$ and $\sigma_{\alpha}=\frac{\pi}{180} \mathrm{rad}$. The measurements are taken at a frequency of $5 \mathrm{~Hz}$. That is, the sample period is $T_{s}=0.2 \mathrm{~s}$.

\subsection{Implementation of the Filters}

\subsubsection{The EKF}

Before we implement the EKF, the fundamental matrix $\phi$, the system dynamic matrix $F$, the observational matrix $H$, and their covariance matrices $Q$ and $R$ have to be derived. These matrices are to be updated at each time step.

The matrix $F$, is the Jacobian of the system dynamics matrix $f(\cdot)$ and is computed by taking the partial derivative of $f(\cdot)$ with respect to $X$,

$$
F=\left.\frac{\partial f(X)}{\partial X}\right|_{X=\hat{X}}=\left[\begin{array}{ccc}
0 & 0 & -v \sin (\theta) \\
0 & 0 & v \cos (\theta) \\
0 & 0 & 0
\end{array}\right]
$$

The fundamental matrix $\phi_{k}$ is approximated by using the Taylor-series expansion of $e^{F T_{s}}$

$$
\phi_{k}=I+F T_{s}+\frac{F^{2} T_{s}^{2}}{2 !}+\frac{F^{3} T_{s}^{3}}{3 !}+\cdots
$$

where $T_{s}$ represents the sample period and $I$ is the identity matrix. Generally, only the first two terms of Eqn.(3.5) are chosen to simplify the derivation and reduce the 
computational cost. The fundamental matrix has the form:

$$
\phi_{k} \approx I+F T_{s}=\left[\begin{array}{ccc}
1 & 0 & -v T_{s} \sin (\theta) \\
0 & 1 & v T_{s} \cos (\theta) \\
0 & 0 & 1
\end{array}\right]
$$

The discrete process noise $Q_{k}$ can be found from the continuous process-noise matrix according to

$$
Q_{k}=\int_{0}^{T_{s}} \phi(\tau) Q \phi^{T}(\tau) \mathrm{d} t
$$

where the expressions of $Q$ and $\phi(\tau)$ have the following forms

$$
Q=\left[\begin{array}{ccc}
\sigma_{x}^{2} & 0 & 0 \\
0 & \sigma_{y}^{2} & 0 \\
0 & 0 & \sigma_{\theta}^{2}
\end{array}\right] \quad \phi(\tau)=\left[\begin{array}{ccc}
1 & 0 & -v \tau \sin (\theta) \\
0 & 1 & v \tau \cos (\theta) \\
0 & 0 & 1
\end{array}\right]
$$

Finally, after integration the final expression for the discrete process noise matrix is

$$
Q_{k}=\left[\begin{array}{ccc}
\sigma_{\theta} T_{s}^{3} v^{2} \sin (\theta)^{2}+\sigma_{x} T_{s} & -\left(T_{s}^{3} v^{2} \sigma_{\theta} \sin (2 \theta)\right) / 2 & -T_{s}^{2} v \sigma_{\theta} \sin (\theta) \\
-\left(T_{s}^{3} v^{2} \sigma_{\theta} \sin (2 \theta)\right) / 2 & \sigma_{\theta} T_{s}^{3} v^{2} \cos (\theta)^{2}+\sigma_{y} T_{s} & T_{s}^{2} v \sigma_{\theta} \cos (\theta) \\
-T_{s}^{2} v \sigma_{\theta} \sin (\theta) & T_{s}^{2} v \sigma_{\theta} \cos (\theta) & T_{s} \sigma_{\theta}
\end{array}\right]
$$

The matrix $H$ is the Jacobian of the measurement matrix $h(\cdot)$ and is computed by taking the partial derivative of $h(\cdot)$ with respect to $X$, 


$$
\begin{aligned}
H=\left.\frac{\partial h(X)}{\partial X}\right|_{X=\hat{X}} & =\left[\begin{array}{lll}
\frac{\left(x_{k}-x_{L 1}\right)}{\sqrt{d 1}} & \frac{\left(y_{k}-y_{L 1}\right)}{\sqrt{d 1}} & 0 \\
\frac{\left(x_{k}-x_{L 2}\right)}{\sqrt{d 2}} & \frac{\left(y_{k}-y_{L 2}\right)}{\sqrt{d 2}} & 0 \\
\frac{y_{L 3}-y_{k}}{d 3} & \frac{-\left(x_{L 3}-x_{k}\right)}{d 3} & -1
\end{array}\right] \\
d 1 & =\left(x_{k}-x_{L 1}\right)^{2}+\left(y_{k}-y_{L 1}\right)^{2}
\end{aligned}
$$

where $\left(x_{L 1}, y_{L 1}\right),\left(x_{L 2}, y_{L 2}\right)$, and $\left(x_{L 3}, y_{L 3}\right)$ represent the coordinates of the landmark 1, 2 and 3. $\left(x_{k}, y_{k}\right)$ are the coordinates of the robot.

Finally, the EKF can be implemented by using Eqn.(2.58) to (2.62) recursively to compute the robot pose estimation.

\subsubsection{The UKF}

The implementation of the UKF is easier than the implementation of the EKF since it does not require any Jacobian derivations. The UKF is implemented simply following all the steps in section 2.6.2.

The UKF uses $n^{a}=19$ sigma points to perform pose estimate. The measurement noise in the simulation is introduced in a nonlinear fashion. According to [5], the augmented vector as shown in Eqn.(2.82) has to be expanded to include the observation terms. Therefore, the dimension of the augmented state vector is $n^{a}=n+n_{w}+n_{\eta}=3+3+3=9$. The number of sigma points for this simulation becomes $2 n^{a}+1=19$. The augmentation introduces more sigma points which leads to more computational complexity of the UKF implementation. However, the 
measurement noise is processed by the algorithm with respect to its the mean and covariance and retains the same order of accuracy as the uncertainty in the state vector.

\subsubsection{The Bootstrap PF}

The bootstrap particle filter is performed by Algorithm 2.10 for the robot pose estimation. The simulation is repeated by applying the bootstrap filter with sample sizes of 500, 1000, and 2500 respectively. For each sample size, 50 runs of Monte Carlo simulation are performed to gather sufficient data for statistical analysis.

\subsubsection{Initialization}

The robot was initially positioned at $(400,500)$ with orientation of 0 degree. Hence the initial state vector is

$$
x_{0}=\left(x_{0}, y_{0}, \theta_{0}\right)^{T}=(400,500,0)^{T}
$$

Each simulation trial starts from 0s and goes to 160s. The robot moves along a nonlinear path. The measurements are taken with a sampling period of $T=0.2 \mathrm{~s}$. This means that each simulation trial consists of 800 sampling steps. The units in the simulations are centimeters in length and radian in angle. As shown in Figure 3.3,

the landmark 1, 2 and 3 are positioned at $\left(x_{L 1}, y_{L 1}\right)=(0,0),\left(x_{L 2}, y_{L 2}\right)=(900,0)$, and $\left(x_{L 3}, y_{L 3}\right)=(1000,1000)$ respectively. 
The initial covariance matrix $Q_{0}$ of the process noise is

$$
Q_{0}=\left[\begin{array}{ccc}
\sigma_{x}^{2} & 0 & 0 \\
0 & \sigma_{y}^{2} & 0 \\
0 & 0 & \sigma_{\theta}
\end{array}\right]=\left[\begin{array}{ccc}
1 & 0 & 0 \\
0 & 1 & 0 \\
0 & 0 & (\pi / 180)^{2}
\end{array}\right]
$$

The covariance matrix $R$ of the measurement noise is

$$
R=\left[\begin{array}{ccc}
\sigma_{r 1}^{2} & 0 & 0 \\
0 & \sigma_{r 2}^{2} & 0 \\
0 & 0 & \sigma_{\alpha}^{2}
\end{array}\right]=\left[\begin{array}{ccc}
2^{2} & 0 & 0 \\
0 & 2^{2} & 0 \\
0 & 0 & (\pi / 180)^{2}
\end{array}\right]
$$

The error covariance of initial pose estimation is

$$
P_{0}=\left[\begin{array}{ccc}
1 & 0 & 0 \\
0 & 1 & 0 \\
0 & 0 & (\pi / 180)^{2}
\end{array}\right]
$$

\subsection{Results}

In the simulations, we applied the non-linear filters to the model of Section 3.2.1 and 3.2.2 for performance comparison. The EKF, UKF, and the bootstrap filter were implemented in Matlab on a $2.4 \mathrm{GHz}$ Intel Core 2 Quad processor. 50 runs of Monte Carlo simulation were performed on each filter to gather sufficient data. All filters were applied to track the system state $X_{k}=\left(x_{k}, y_{k}, \theta_{k}\right)^{T}$, where $x_{k}$ and $y_{k}$ specify the coordinates of the position of the robot in the Cartesian plane; $\theta_{k}$ denotes the angle 
between the longitude of the robot and the X-axis on the Cartesian plane.

As described earlier in this section, both the process model and the measurement model are non-linear functions. The mobile robot moves along highly non-linear trajectories which are composed of straight lines and circles as shown in Figure 3.4. Two measurements including the distance $r$ and the bearing $\alpha$ are available at each time step. Figure 3.5 and 3.6 illustrate RMSE of positions and headings for each

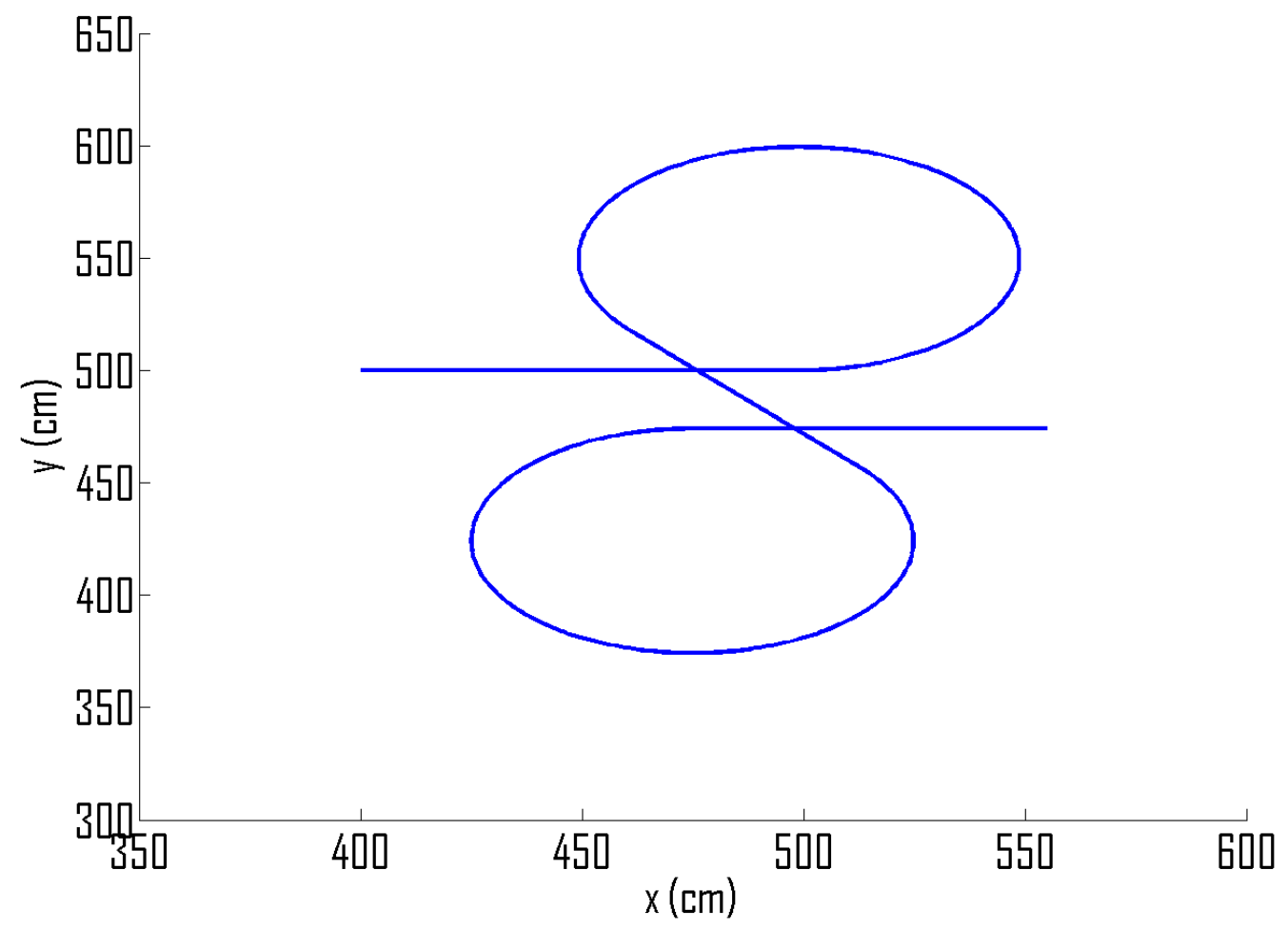

Figure 3.4: The real path of Mobile Robot (solid line).

non-linear filter.

We perform 50 trials of the Monte Carlo simulations for the EKF, the UKF and the bootstrap filter (sample size: 500, 1000, 2500) respectively. Each simulation trial consists of $N=800$ epochs. The average RMSE (the root mean square difference 


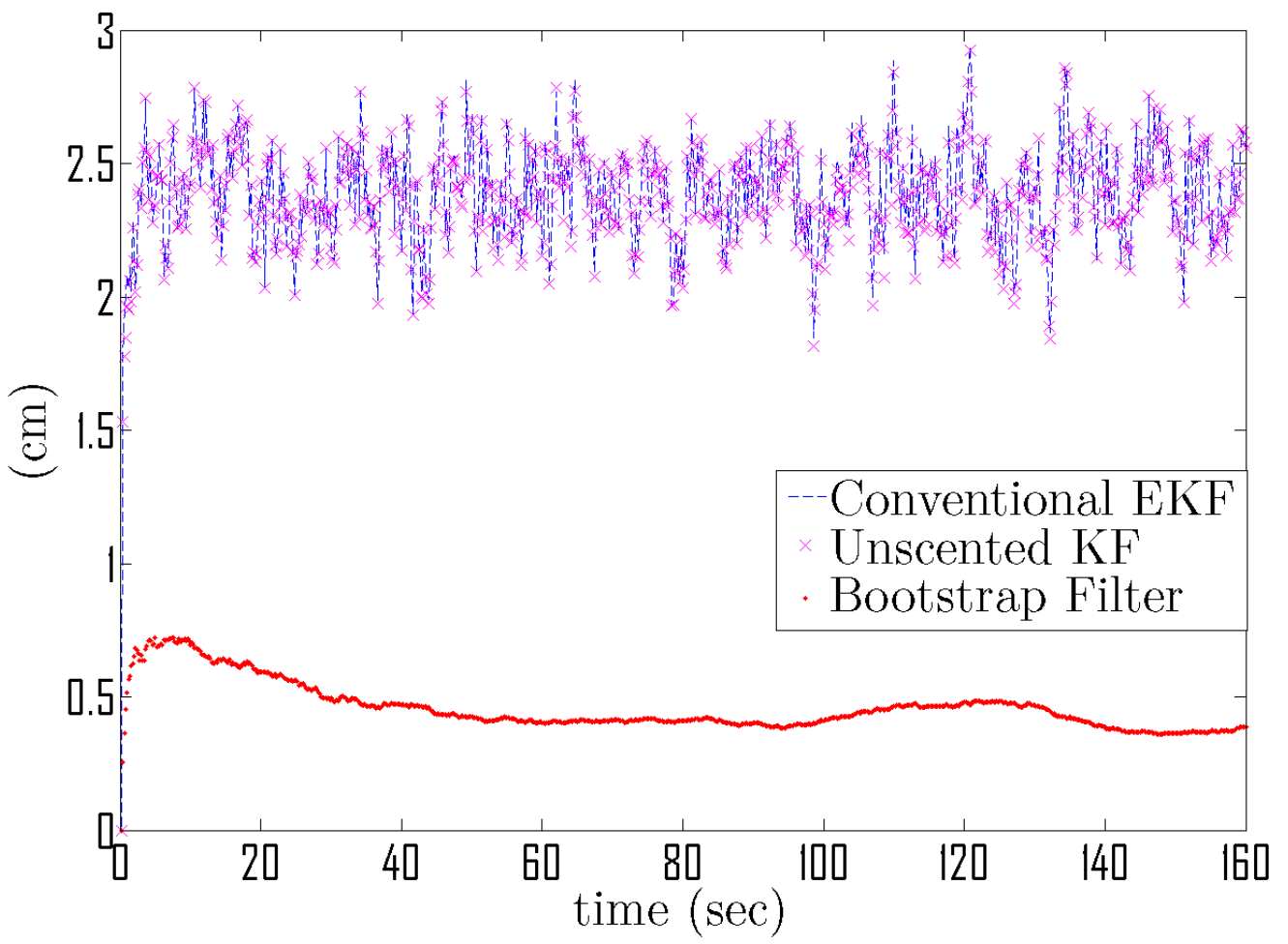

Figure 3.5: A comparison of RMS errors of position: EKF,UKF,Bootstrap Filter $(2500$ samples $)$

between the true values and the state estimates) are computed by the following equation:

$$
R M S E=\frac{1}{n} \sum_{j=1}^{n} \sqrt{\frac{1}{N} \sum_{i=1}^{N}\left(x_{j}^{i}-\hat{x}_{j}^{i}\right)^{2}}
$$

where:

- $x_{j}^{i}$ specifies the true system state at the time step $j$ of $i$ th simulation. $(j=1, \ldots, N$; $i=1, \ldots, n)$.

- $\hat{x}_{j}^{i}$ is the system state at the time step $j$ of $i$ th simulation.

- System state index: $j=1, \ldots, N$,(In our case, $N=800$ ).

- Simulation trial index: $i=1, \ldots, n$, (In our case, $n=50$ ).

The average RMSE and the variance with respect to the position and orientation are evaluated. The comparison for RMSE in position are given in Table 3.1 and 


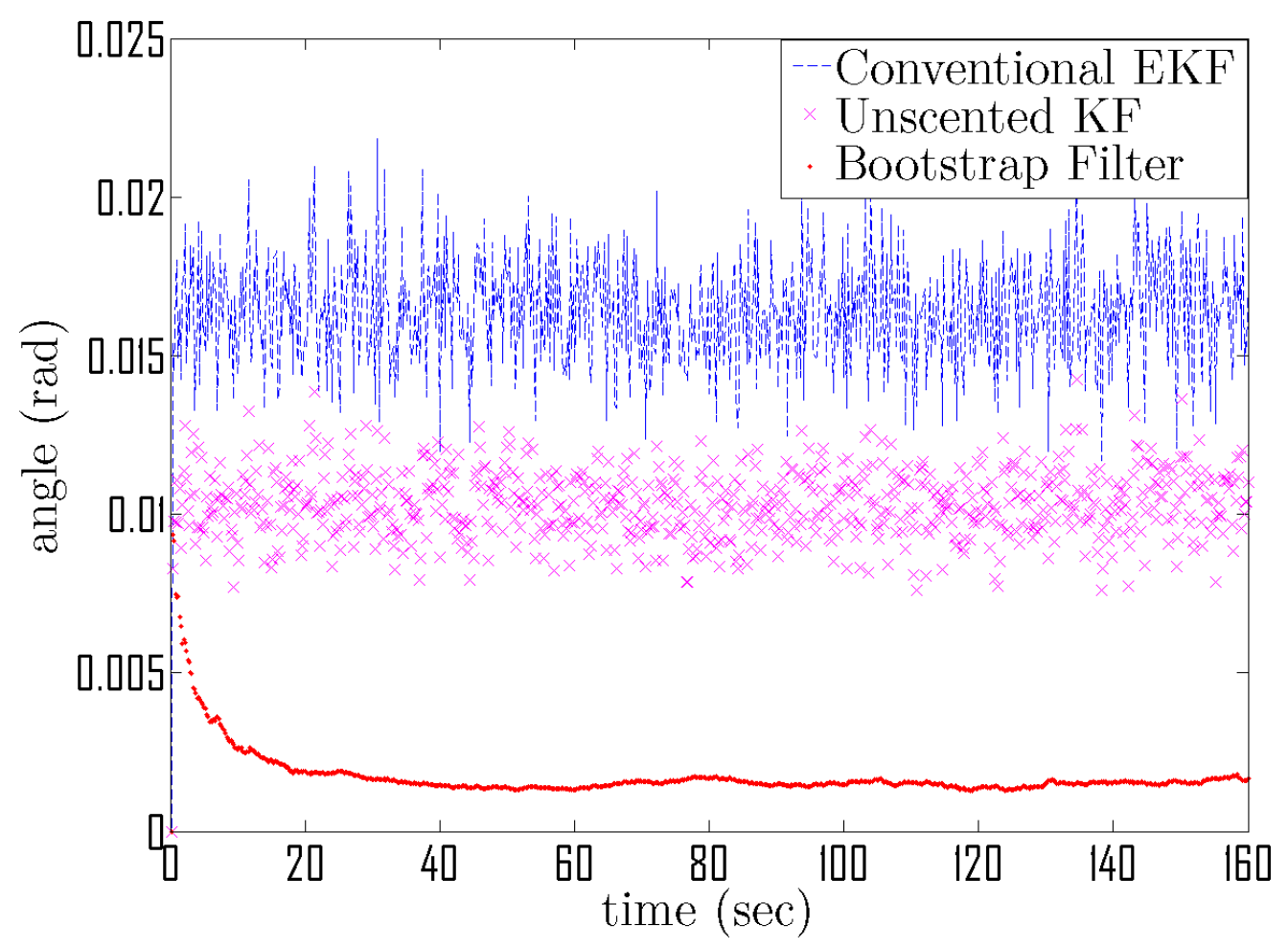

Figure 3.6: A comparison of RMS errors of heading: EKF,UKF,Bootstrap Filter(2500 samples)

illustrated in Figure 3.7 and Figure 3.8. The comparison of RMSE in orientation is given in Table 3.2 and illustrated in Figure 3.9 and Figure 3.10. The computational complexity of each filter is given in Table 3.3 and illustrated in Figure 3.11. As described in Section 3.3.2, the UKF implementation requires more sigma points and leads to more computational complexity. Thus, the computational time of the UKF is more than 10 times over the EKF and it is longer than the theoretical value.

From Table 3.1 and 3.2, we can see that the EKF provides the worst performance comparing to the other two filters. The UKF has slightly better performance than the EKF on state estimate of position. However, it uses more than 10 times the computational cost than the EKF. In our case, the EKF is definitely a better choice over the UKF on robot pose estimation.

It is also clearly noticeable that the bootstrap filter has a much smaller RMSE 
Table 3.1: Averaged RMS Position Error in Centimeters (50 Trials)

\begin{tabular}{|c|c|c|}
\hline Algorithm & RMSE-Position & VAR \\
\hline EKF & 2.3864 & 0.0077 \\
\hline UKF (19 Sigma Points) & 2.3748 & 0.0076 \\
\hline Bootstrap Filter $(\mathrm{n}=500)$ & 1.0587 & 0.12931 \\
\hline Bootstrap Filter $(\mathrm{n}=1000)$ & 0.8080 & 0.05390 \\
\hline Bootstrap Filter $(\mathrm{n}=2500)$ & 0.4433 & 0.02223 \\
\hline
\end{tabular}

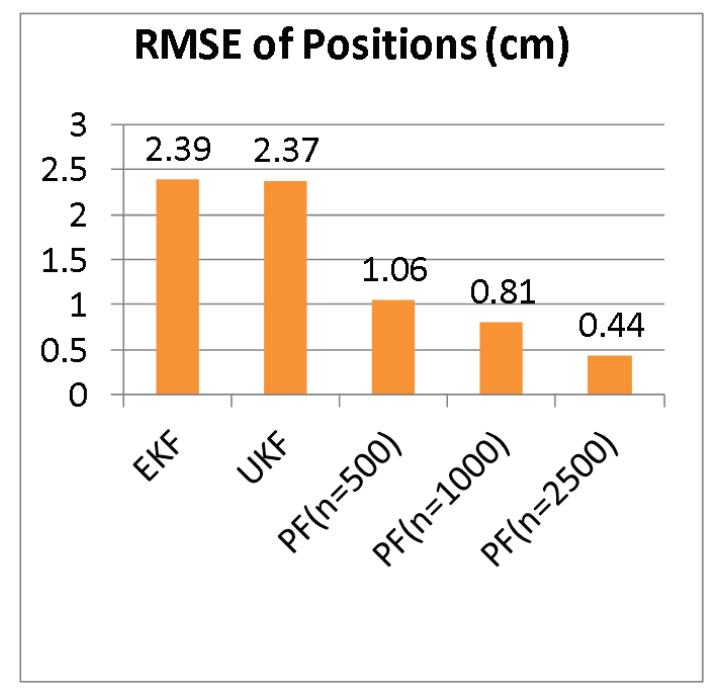

Figure 3.7: RMSE on Position Estimation

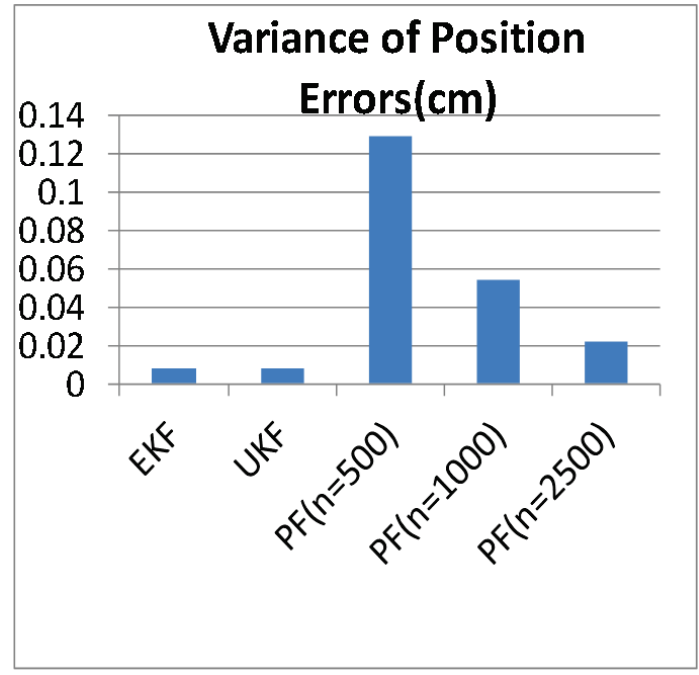

Figure 3.8: Variance of RMSE on Position Estimation

Table 3.2: Averaged RMS Heading Error in Centimeters (50 Trials)

\begin{tabular}{|c|c|c|}
\hline Algorithm & RMSE-Heading & VAR \\
\hline EKF & 0.0164 & $1.5576 \mathrm{E}-7$ \\
\hline UKF & 0.0103 & $1.3545 \mathrm{E}-7$ \\
\hline Bootstrap Filter $(\mathrm{n}=500)$ & 0.0033425 & $3.4369 \mathrm{E}-7$ \\
\hline Bootstrap Filter $(\mathrm{n}=1000)$ & 0.0031204 & $1.8517 \mathrm{E}-7$ \\
\hline Bootstrap Filter $(\mathrm{n}=2500)$ & 0.0018395 & $1.0983 \mathrm{E}-7$ \\
\hline
\end{tabular}




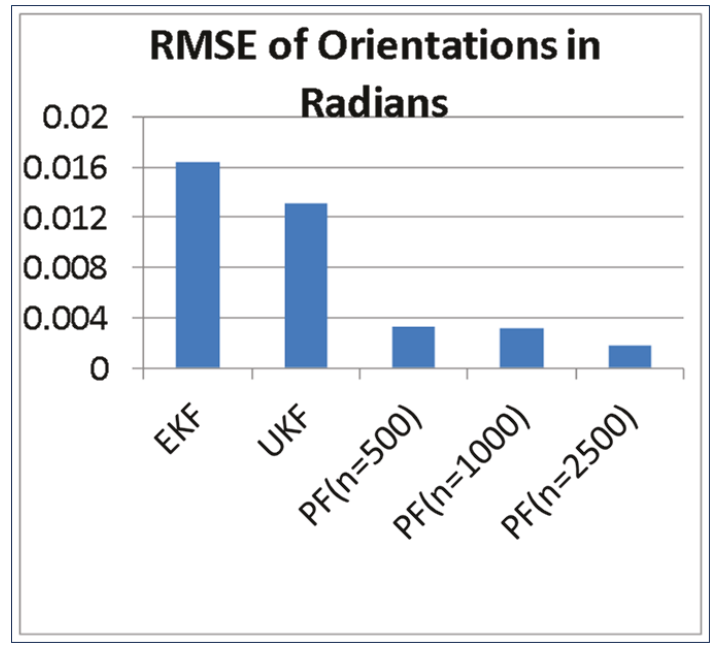

Figure 3.9: RMSE on Orientation Estimation

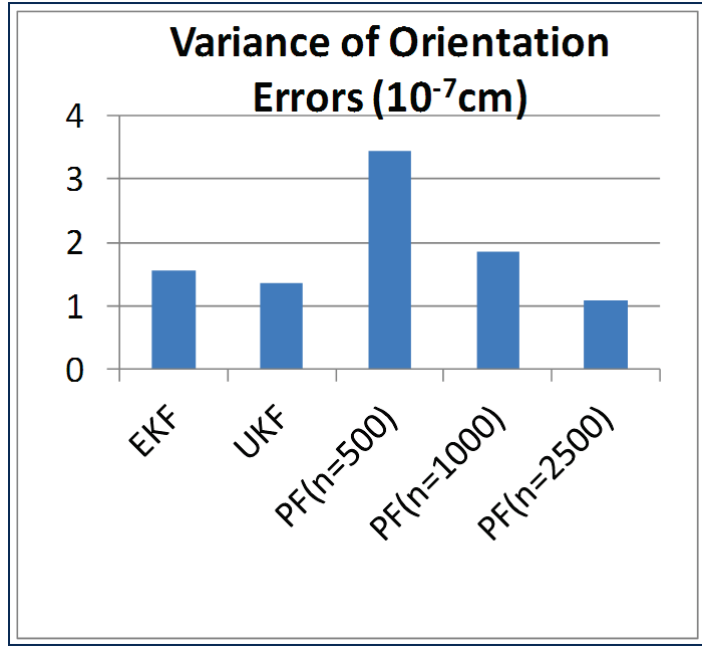

Figure 3.10: Variance on Orientation Estimation

Table 3.3: Computational Complexity (50 Trials)

\begin{tabular}{|c|c|c|}
\hline Algorithm & Time $(\mathrm{s})$ & Relative \\
\hline EKF & 1.5203 & 1.00 \\
\hline UKF & 26.3848 & 17.36 \\
\hline Bootstrap PF $(\mathrm{n}=500)$ & 866.23 & 569.78 \\
\hline Bootstrap PF $(\mathrm{n}=1000)$ & 1738.13 & 1143.28 \\
\hline Bootstrap PF $(\mathrm{n}=2500)$ & 4378.73 & 2880.18 \\
\hline
\end{tabular}

both on the position and orientation estimates in comparison to the EKF and UKF. Although the bootstrap filter leads to a much higher quality of state estimation, it takes around 860 seconds to perform the pose estimate (500 particles) of a trial with 160-sec robot navigation time. In order to implement the bootstrap particle filter in practice, it would be feasible to use parallel computation to implement a bootstrap $\mathrm{PF}$ in a high-level language such as $\mathrm{C}++$.

Figure 3.12 illustrates the performance comparison of the bootstrap filter with 500, 1000, 2500 samples. It shows that the average RMSE decreases as the number of samples increases. This is another advantage of the particle filter: unlike the EKF 


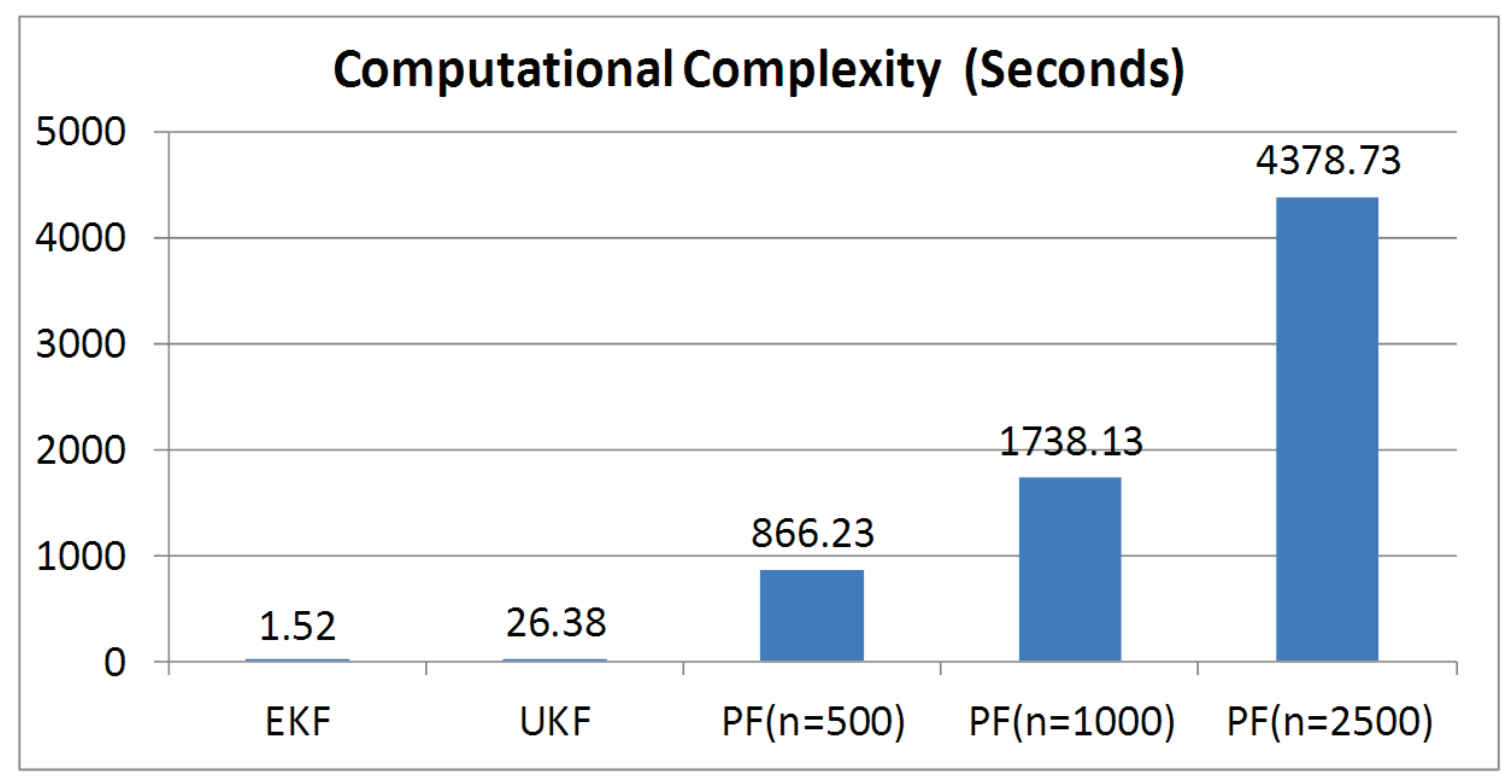

Figure 3.11: A comparison of Computational Complexity of the EKF, UKF and Bootstrap Filter with 500,1000,2500 particles

and the UKF, the performance of the particle filter can be tuned by adjusting the number of samples. We finally conclude that the bootstrap filter is the best filter if the computational cost is not our primary concern. However, if the computation cost is critical, the EKF is considered as the most efficient approach which also provides a relatively good performance.

\subsection{Chapter Summary}

This chapter presented an application for mobile robot pose estimation. The pose estimation was implemented by using the nonlinear filters: the EKF, UKF and the bootstrap particle filter. The EKF was implemented by linearizing the system functions with the Taylor-series expansion to approximate the posterior PDF. Although the EKF implementation needs to take extra effort to derive Jacobian matrices which is also error-prone, it is the most efficient approach with the least computational 


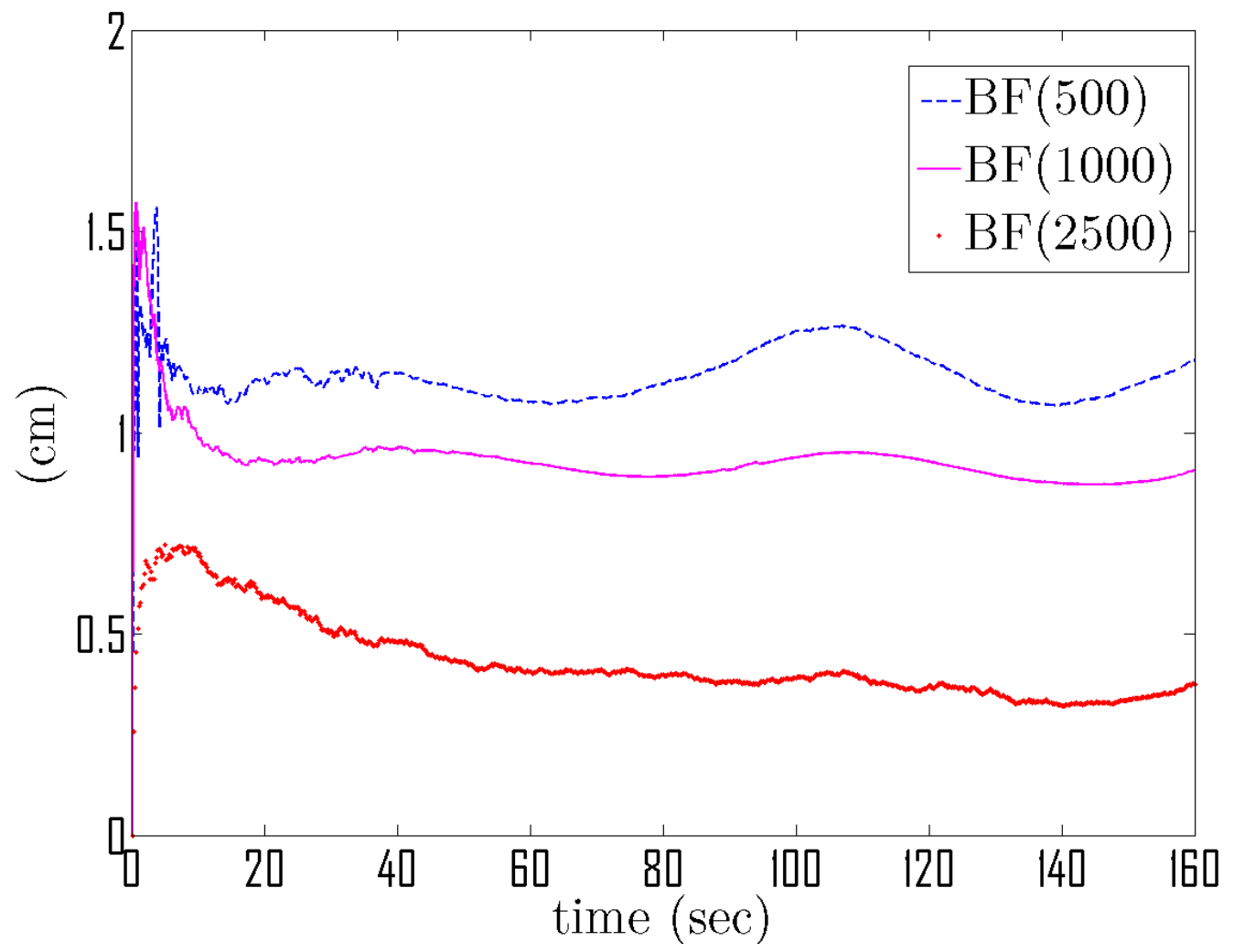

Figure 3.12: A comparison of RMS errors for position of the EKF and Bootstrap Filter with 500,1000,2500 samples

complexity among the three filters. The UKF and the bootstrap particle filter approximate the PDF in different ways. The UKF uses a set of deterministic number of samples and has nearly the same state estimation accuracy to the EKF. However it needs more than 10 times the computational effort than the EKF. The bootstrap particle filter has the best state estimation accuracy and the highest computational cost. The computational cost for the particle filter depends on its sample size. In our simulation where the sample size was chosen between 500 and 2500, the computational cost of the bootstrap PF was 500 to 3000 times more than that of the EKF.

The performance of the EKF and UKF is difficult to tune. This is another advantage of the particle filter. The performance of the particle filter can be tuned by 
adjusting the number of particles. If a parallel implementation is feasible, we can finally conclude that the particle-based filter is the best choice among the three nonlinear filtering approaches. However, the EKF is certainly the best choice when the computational complexity is a critical concern such as in real time applications. 


\section{Chapter 4}

\section{Experiment and Results for Pose Estimation}

\subsection{Introduction}

A robot experimental platform is necessary and useful at evaluating the performance of pose estimation. An experimental platform which is called the Mobile Cooperating Robot Test Platform(MCRTP) is utilized in our experiments to perform pose estimation. The MCRTP is a low cost, scalable, cooperative robot test system. It consists of the Arduino-based differential robots and a camera system. The camera system is a combination of a USB digital camera and a software application. It can track and broadcast robot positions over a wireless network.

\subsection{Architecture}

The MCRTP has a client-server architecture. The camera system and a computer are classified as the server. One or more mobile robots are thought of as the remote workstations. The camera system is composed of a USB digital camera and a software application running on a computer. The camera is fixed at the ceiling on the top of 
experimental area. It is connected to the computer with a USB cable. The Arduinobased mobile robot embeds a XBee receiver which allows its actuator and sensors to be accessed remotely through a wireless network. Figure 4.1 presents the architecture of the experimental platform.

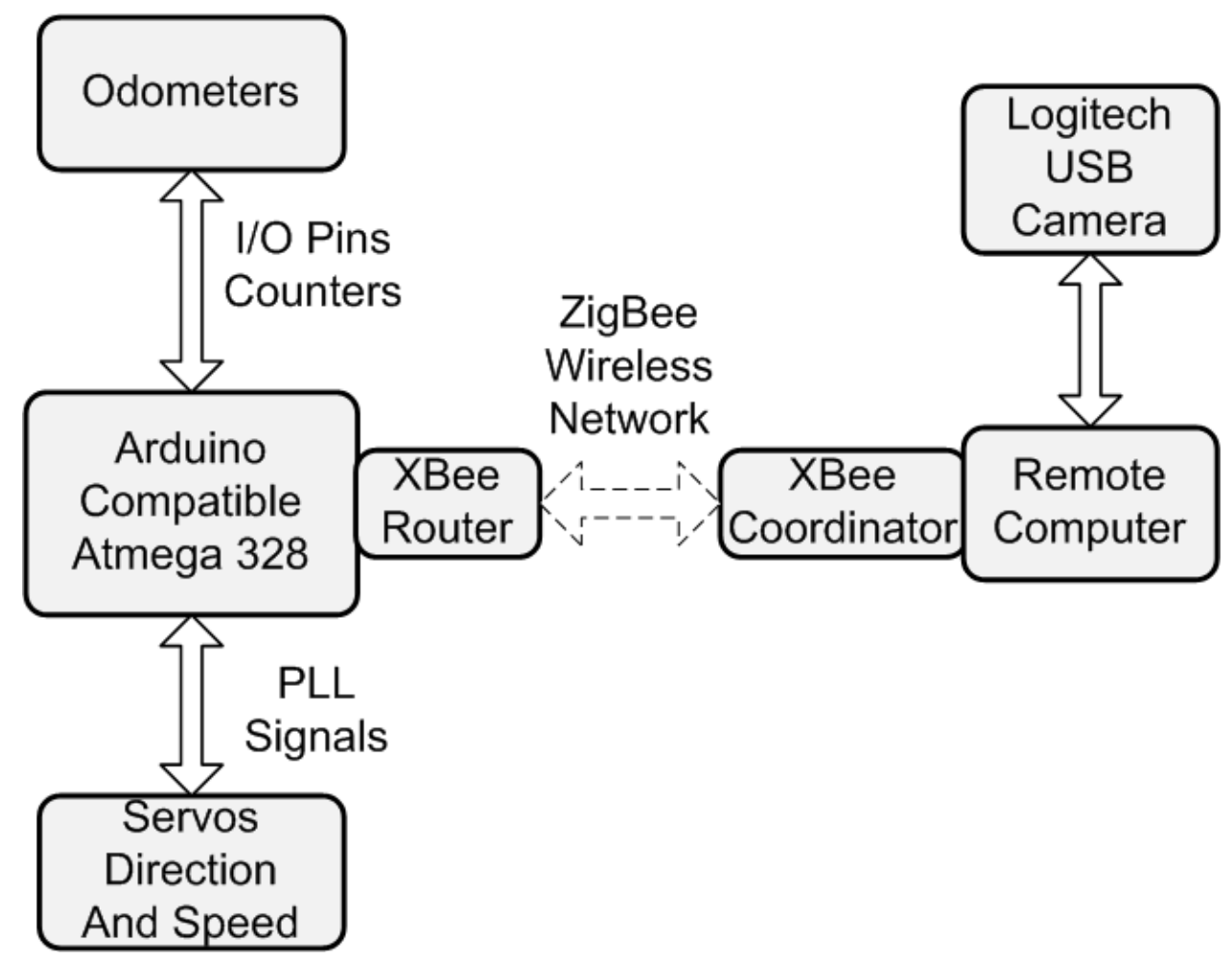

Figure 4.1: The Architecture of the Experimental Platform

The mobile robot in the MCRTP is controlled by Romeo-All in one Controller, an Arduino compatible microcontroller. The robots are small, two-wheeled, battery powered robots. Arduino is an open source computing platform designed specifically for running external devices such as motors and sensors [45]. The Arduino compatible board uses an Atmega328 microcontroller. The board is powered by one 7.4v LiPo(1600mAh) rechargeable battery and the motors (298:1 Micro Metal Gear Motor) are rated at $6 \mathrm{~V}$ with a 298:1 gear ratio.

As illustrated in Figure 4.1, the computer exchanges data with the mobile robots through a ZigBee wireless network. In order to enable wireless communication, the 
computer and the robots are both equipped with XBee communication chips. A XBee chip is running in router mode and connected to the computer externally through a USB port.

Figure 4.2 shows one of the robots equipped with an LCD display. The key hardware components of the robots are the Arduino microcontroller, the motor shield, and the XBee chip.

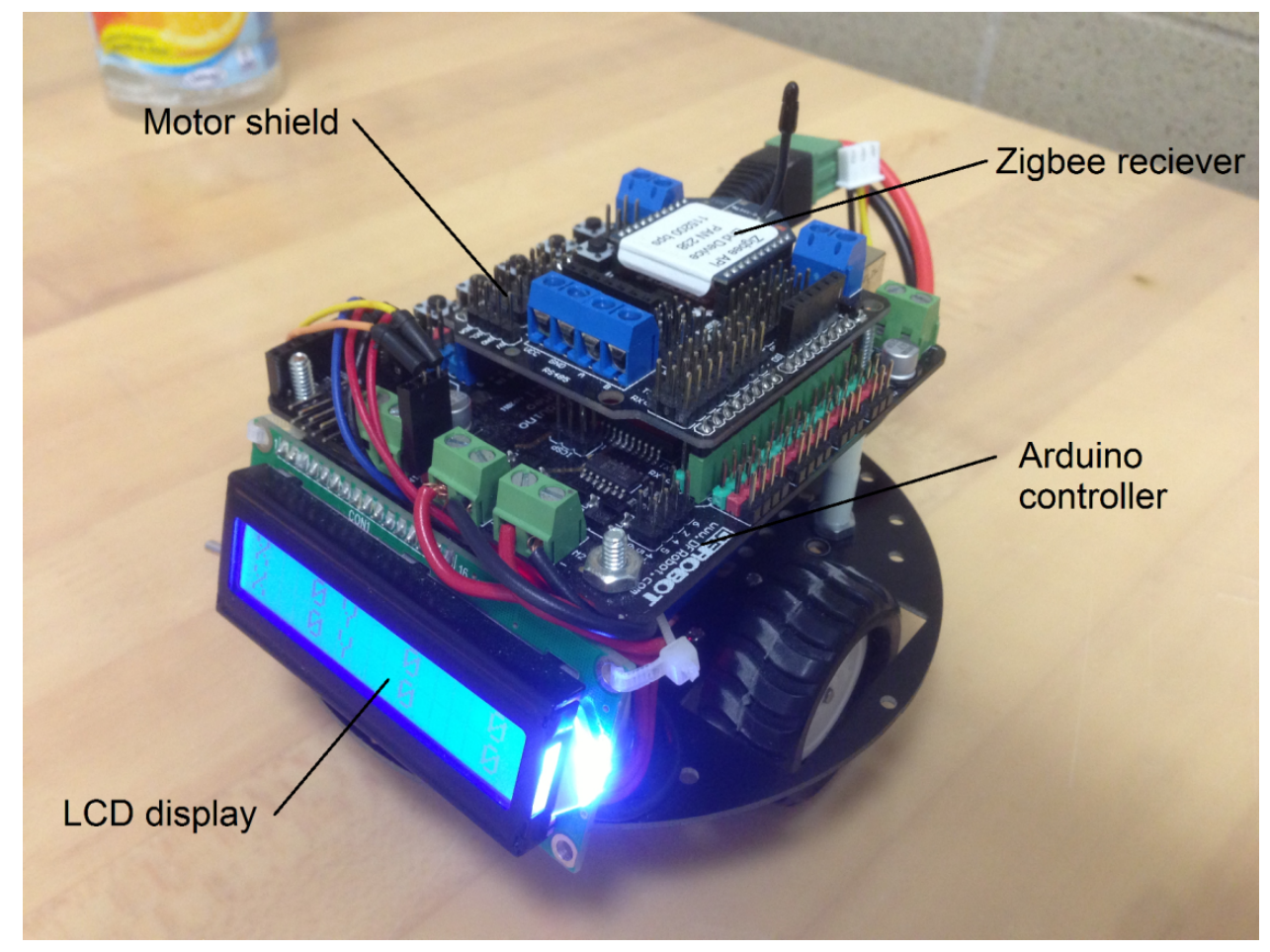

Figure 4.2: A 2-wheeled mobile robot with a front-facing LCD display

In order to track the motions of the mobile robots, a vision system is running on the computer of the experimental platform. The vision system is a software application developed in $\mathrm{C}++$ and it is running on the Windows operating systems. The vision system makes use of a USB camera to locate the robots and communicates with the robots through a XBee transmitter. The USB camera (Logitech HDc615) can record images with a $640 \times 480$ frame size and visually tracks the positions of the robots.

The tracking of the robots is accomplished by a software, namely, Open Computer 
Vision Library (OpenCV). The OpenCV software library can filter images by colours. The colours are identified in the OpenCV software library by using a HSV (hue, saturation, value) value. Each robots is equipped with a rectangular colour identifier. The OpenCV software library employs an algorithm, namely, Canny edge detection to identify the rectangular color identifier of a robot. The algorithm first applies a Gussian blur to the image to reduce noise, then each pixel is examined and tested if it is part of a gradient(i.e. a change in colour). The gradient direction is determined based on surrounding pixels. An edge is detected on the basis of a gradient direction. Finally, the positions of the robot are identified individually with respect to its colour identifier by the OpenCV software library.

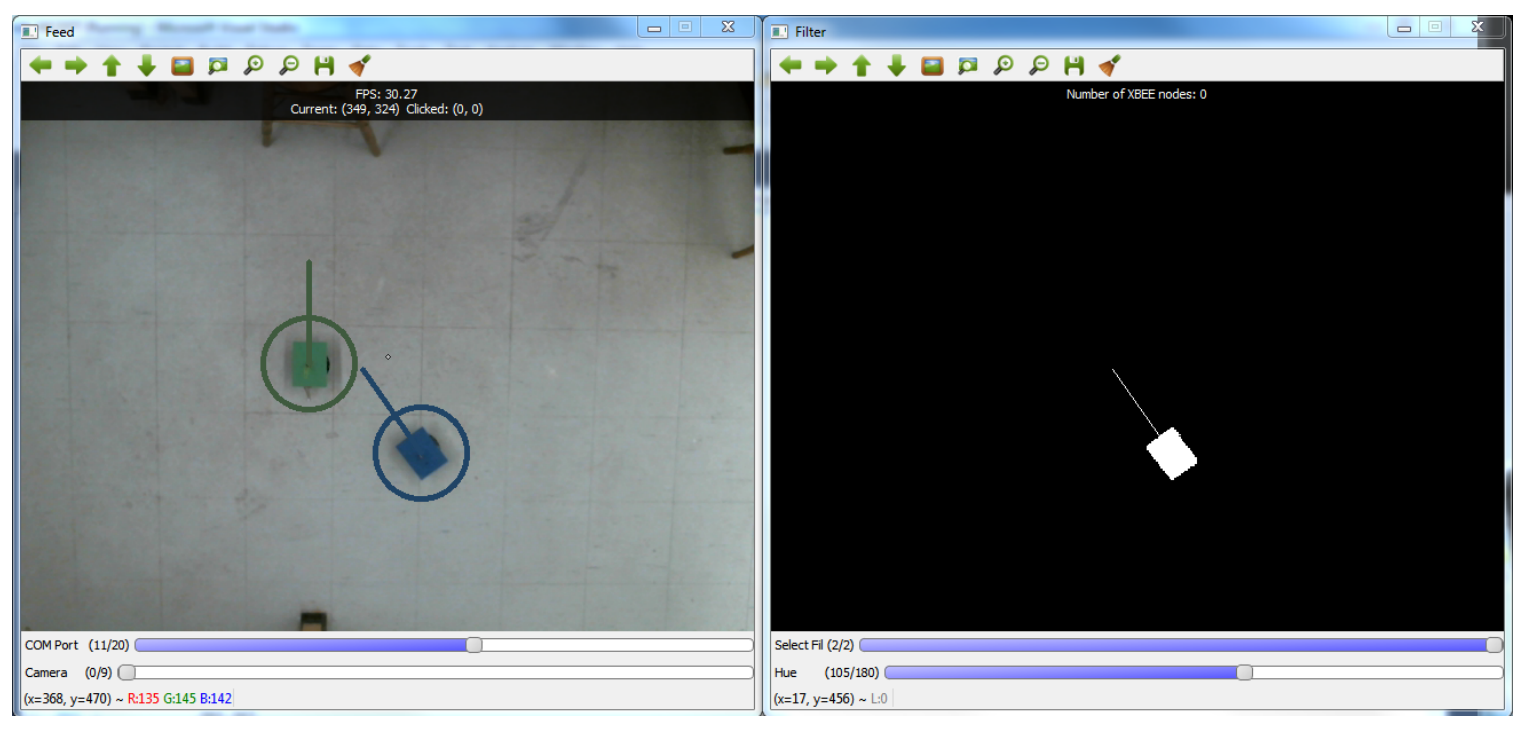

Figure 4.3: Screenshot of the OpenCV vision system, On the left robots is identified by colour. On the right is the filtered view for an individual colour.

The computer in the MCRTP system has a $3.3 \mathrm{GHz}$ Intel Core $\mathrm{i} 3$ processor and $4 \mathrm{G}$ RAM. The operating system is Windows 7 32-bit. It has enough computing power to efficiently implement all tasks including the software tasks and the external hardware tasks in our experiments. 


\subsection{The Mobile Robot and Its Actuator}

The MCRTP uses Arduino-based autonomous robots in experiments. The robots are differential two wheeled robots that are steered by spinning the wheels at different speeds with respect to one another. The left and right wheels of a robot are driven by two different motors. The motor speed is controlled by the digital input, a duty cycle value written to the motor pins. The speeds of the left and right wheels are scalars between 0 and 255. Each duty cycle scalar is relative to 127 (both wheels are stopped), and scalars less than 127 spin the wheel backwards, and scalars greater than 127 spin the wheel forwards.

Notice that the robots are running an open loop system in experiments. That is, there is no feedback from the system states with respect to the effect of the programmed actions. For example, the robot may not run in a straight path even though the same input are applied to the motors that drive the left and the right wheels. This of course is the expected result due to the process noise.

There are many factors that contribute to the process noise including the battery strength, the traction of the surface, the difference between the left and the right wheels of the robots. Moreover, two motors that drive the two robot wheels cannot produce the same noise and torques even if they are applied to the input of the same voltage. First the sizes of the wheels of the robots are usually slightly different from the manufacturer standard. The motors cannot have the same output with the input. These would cause calculation errors of the robot's speed. Second, since the sizes of the two wheels on each robot cannot be the same exactly, it leads to a calculation error of the robot's turning angle. The wheels of a robot also may slip on the ground during the experiment. Finally, battery strength is probably one of the most significant factors determining the torque on the wheels. All the above errors may lead to inconsistent results of the robot's actual displacements. 
The mobile robots are equipped with gear-motors with $298: 1$ metal gearbox which has a Free-Run speed of $100 R P M$ at $6 \mathrm{~V}$. The circumference of robot wheels is $131.95 \mathrm{~mm}$. Thus the maximum speed of a mobile robot along a straight path can be calculated as $0.7215 \mathrm{ft} / \mathrm{sec}$ and $22.0 \mathrm{~cm} / \mathrm{sec}$. A sample interval $T_{0}<=100 \mathrm{~ms}$ (corresponding to a displacement of less than $3.0 \mathrm{~cm}$ ) would be reasonable.

The speed of the robot is controlled by the digital inputs to the motors attached on left and right wheels. The robot's true movements can be measured by a tape. An experiment is conducted to evaluate the magnitude of the process noise. The robots are programmed to move along straight paths for 10 seconds. For each path, the motors of the robot are written with different forward digital inputs. That is, the lengths of the robot movements are different in 10 seconds. The length of each path is measured by a tape. The experiment was repeated 5 times on each path with the same forward input on the motors. The means and errors of each path is calculated and shown in Table 4.1.

Table 4.1: Evaluation of Process Error

\begin{tabular}{|l|l|l|l|l|l|}
\hline $\begin{array}{l}\text { Digital } \\
\text { Input }\end{array}$ & $\begin{array}{l}\text { Real } \\
(\mathrm{cm})\end{array}$ & $\begin{array}{l}\text { Mean } \\
(\mathrm{cm})\end{array}$ & $\begin{array}{l}\text { Error } \\
(\mathrm{cm})\end{array}$ & $\begin{array}{l}\text { Max Error } \\
(\mathrm{cm})\end{array}$ & $\begin{array}{l}\text { Max Error } \\
(\%)\end{array}$ \\
\hline 20 & 31.1 & 31.8 & 0.7 & 1.5 & 4.8 \\
\hline 40 & 69.2 & 70.6 & 1.4 & 2.1 & 3.0 \\
\hline 80 & 140.2 & 141.6 & 1.4 & 3.4 & 1.0 \\
\hline 100 & 177.2 & 174.5 & 2.7 & 6.3 & 3.6 \\
\hline
\end{tabular}

The process error can be calculated from the difference between the actual displacements and the calculated values. The results on Table 4.1 show that the process error is relatively consistent in the tests. The max process error is about $5 \%$ of the length of path. Taking some outliers into consideration, $6 \%$ error of the total length of the trajectory would be a fair value for the process error in our experiment. Finally, 
we set the process error in our experiment as $\sigma_{v_{L}}=\sigma_{v_{R}}=1.0 \mathrm{~cm} \approx 2.5$ pixels. We assume that all process errors are additive Gaussian white noise.

\subsection{The Sensor of the Measurement System}

As described in 4.2, the task of the camera system in the MCRTP is to track the mobile robots. The camera system is composed of a digital camera and a software application. The digital camera is a Logitech HDc615 webcam set which is capable of recording images with a $640 \times 480$ frame size. The digital images can be captured and sent back to the computer periodically during the experiment. The images are processed by OpenCV to generate the filtered images with respect to the robot's coloured identifier. Finally, the mobile robot's real-time positions and orientations are calculated.

In order to evaluate the noise of the camera system, we conducted another experiment by comparing the real measurements by a ruler with the readings from the camera system. The experiment was conducted by dividing the camera frame equally into $35(5 \times 7)$ squares and each has a size of 1 foot in square. The pixels in $\mathrm{x}$ and $\mathrm{y}$ directions of each square are recorded. If we assume the square in the middle of frame is perfect and without distortion, the maximum error can be calculated by comparing the difference of the number of pixels in $\mathrm{x}$ and $\mathrm{y}$ directions of each square with the number of pixels of the assumed perfect square.

The maximum error in $\mathrm{x}$-direction yielded from the experiments is about \pm 9 pixels (corresponding to $3.5 \mathrm{~cm}$ ). Whereas the maximum error in $\mathrm{y}$-direction is about \pm 3 pixels (corresponding to $1.2 \mathrm{~cm}$ ). The total error yielded in the experiment would be less than $4 \mathrm{~cm}$ and the camera has an accumulative positional accuracy of over $96 \%$. In this case, we can set the measurement errors $\eta_{x}$ and $\eta_{y}$ with respect to the movements along the $x$ and $y$ directions as $\eta_{x}=4 \mathrm{~cm} \approx 10$ pixels and $\eta_{y}=1.2 \mathrm{~cm} \approx 3.0$ pixels. In 
our experiments, we noticed that the noise of the camera system is relatively small. Moreover, most of the measurement errors are produced by the distortion of the lens which has non-Gaussian distribution. In order to compare the performance of the filters with respect to the measurement errors with Gaussian distribution, white noise is added into each measurement deliberately in the experiments to evaluate the performance of all non-linear filters with respect to the measurement noise.

\subsection{System Dynamics Modelling}

In order to apply the non-linear filters to perform pose estimate, the system dynamic model of the mobile robots is required. The derivation of the system dynamic model is based on Figure 4.4. In Figure 4.4, the term $D$ denotes the instantaneous curvature

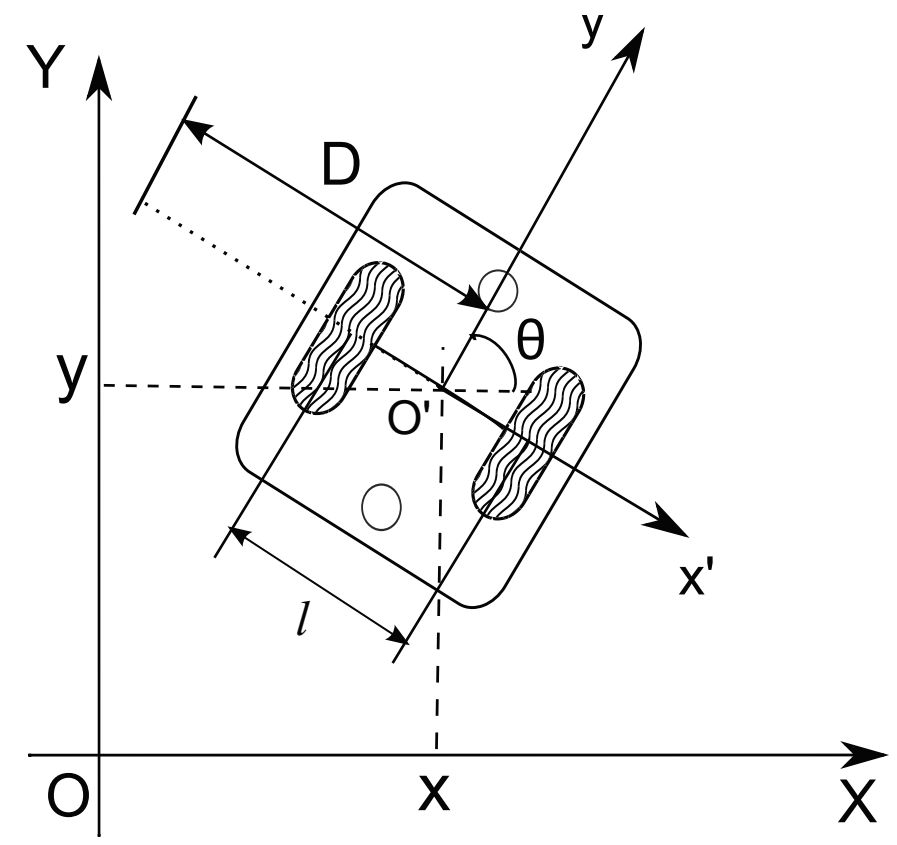

Figure 4.4: Kinematic Model of a Differential Drive Mobile Robot.

radius of the robot trajectory with relation to the center of the axis. The term $l$ specifies the distance between axles of the two wheels. In our experiments, the length 
$l$ is equal to $3.7 \mathrm{~cm}$. Let $v_{R}$ and $v_{L}$ be the linear velocities of the right and left wheel respectively. Analytically, these linear velocities can be determined as

$$
\begin{aligned}
& v_{R}=\left(D+\frac{l}{2}\right) \omega \\
& v_{L}=\left(D-\frac{l}{2}\right) \omega
\end{aligned}
$$

where $\omega$ is the the angular velocity of the robot. Solving for $\omega$ yields:

$$
\begin{aligned}
& \omega=\frac{v_{R}}{D+\frac{l}{2}} \\
& \omega=\frac{v_{L}}{D-\frac{l}{2}}
\end{aligned}
$$

and set Eqn. (4.3) equal to Eqn. (4.4):

$$
\begin{aligned}
& \frac{v_{R}}{D+\frac{l}{2}}=\frac{v_{L}}{D-\frac{l}{2}} \\
\Rightarrow \quad & v_{R}\left(D-\frac{l}{2}\right)=v_{L}\left(D+\frac{l}{2}\right) \\
& D\left(v_{R}-v_{L}\right)=\frac{l}{2}\left(v_{R}+v_{L}\right) \\
\therefore \quad & D=\frac{l}{2} \times \frac{v_{R}+v_{L}}{v_{R}-v_{L}}
\end{aligned}
$$

substituting Eqn. (4.5) back into Eqn.(4.3) yields:

$$
\begin{aligned}
\omega & =\frac{v_{R}}{\left(\frac{l}{2} \times \frac{v_{R}+v_{L}}{v_{R}-v_{L}}\right)+\frac{l}{2}} \\
& =\frac{v_{R}}{\frac{l}{2}\left(\frac{v_{R}+v_{L}}{v_{R}-v_{L}}+\frac{v_{R}-v_{L}}{v_{R}+v_{L}}\right)} \\
\therefore \omega & =\frac{1}{l} v_{R}-\frac{1}{l} v_{L}
\end{aligned}
$$


Finally, substituting (4.5) and (4.6) into $v=D \omega$ yields:

$$
v=\frac{1}{2}\left(v_{R}+v_{L}\right)
$$

The stochastic differential equations of the kinematic model for the differential drive mobile robots are:

$$
\begin{aligned}
\dot{X}= & f(X)+w \\
= & {\left[\begin{array}{l}
\dot{x} \\
\dot{y} \\
\dot{\theta}
\end{array}\right]=\left[\begin{array}{l}
v \cos \theta \\
v \sin \theta \\
\omega
\end{array}\right]+\left[\begin{array}{l}
w_{x} \\
w_{y} \\
w_{\theta}
\end{array}\right] } \\
= & {\left[\begin{array}{c}
\frac{1}{2}\left(v_{R}+v_{L}\right) \cos \theta \\
\frac{1}{2}\left(v_{R}+v_{L}\right) \sin \theta \\
\frac{1}{l} v_{R}-\frac{1}{l} v_{L}
\end{array}\right]+\left[\begin{array}{c}
w_{x} \\
w_{y} \\
w_{\theta}
\end{array}\right] } \\
\dot{X}= & {\left[\begin{array}{ll}
\frac{1}{2} \cos \theta & \frac{1}{2} \cos \theta \\
\frac{1}{2} \sin \theta & \frac{1}{2} \sin \theta \\
\frac{1}{l} & -\frac{1}{l}
\end{array}\right]\left[\begin{array}{ll}
\frac{1}{2} \cos \theta & \frac{1}{2} \cos \theta \\
v_{R} \\
v_{L}
\end{array}\right]+\left[\begin{array}{ll}
\frac{1}{2} \sin \theta & \frac{1}{2} \sin \theta \\
\frac{1}{l} & -\frac{1}{l}
\end{array}\right]\left[\begin{array}{l}
w_{v_{R}} \\
w_{v_{L}}
\end{array}\right] }
\end{aligned}
$$

where we have

$$
\begin{aligned}
w_{x} & =\frac{1}{2} \cos \theta\left(w_{v_{R}}+w_{v_{L}}\right) \\
w_{y} & =\frac{1}{2} \sin \theta\left(w_{v_{R}}+w_{v_{L}}\right) \\
w_{\theta} & =\frac{1}{l}\left(w_{v_{R}}-w_{v_{L}}\right)
\end{aligned}
$$


The noisy measurements are of the state directly, thus $H=I_{3}$. The measurement model has the form:

$$
z_{k}=\left[\begin{array}{l}
x_{k} \\
y_{k} \\
\theta_{k}
\end{array}\right]+\left[\begin{array}{l}
\eta_{x} \\
\eta_{y} \\
\eta_{\theta}
\end{array}\right]
$$

\subsection{Implementation of the Filters}

\subsubsection{The EKF}

In order to implement the EKF, the fundamental matrix $\phi$, the system dynamic matrix $F$, the observational matrix $H$, and their covariance matrices $Q$ and $R$ have to be derived. The matrix $F$, is the Jacobian of the system dynamics matrix $f(\cdot)$ and is computed by taking the partial derivative of $f(\cdot)$ with respect to $X$,

$$
F=\left.\frac{\partial f(X)}{\partial X}\right|_{X=\hat{X}}=\left[\begin{array}{ccc}
0 & 0 & -\frac{1}{2}\left(v_{R}+v_{L}\right) \sin (\theta) \\
0 & 0 & \frac{1}{2}\left(v_{R}+v_{L}\right) \cos (\theta) \\
0 & 0 & 0
\end{array}\right]
$$

As explained in Chapter 3, the fundamental matrix $\phi_{k}$ is approximated by using the Taylor-series expansion of $e^{F T_{s}}$ and only the first two term of Eqn.(3.5) are chosen. Thus the fundamental matrix has the form:

$$
\phi_{k} \approx I+F T_{s}=\left[\begin{array}{ccc}
1 & 0 & -\frac{1}{2}\left(v_{R}+v_{L}\right) T_{s} \sin (\theta) \\
0 & 1 & \frac{1}{2}\left(v_{R}+v_{L}\right) T_{s} \cos (\theta) \\
0 & 0 & 1
\end{array}\right]
$$


The discrete process noise $Q_{k}$ is derived from the continuous process-noise matrix [21]:

$$
Q_{k}=\int_{0}^{T_{s}} \phi(\tau) Q \phi^{T}(\tau) \mathrm{d} t
$$

where the expressions of $Q$ and $\phi(\tau)$ have the following forms

$$
Q=\left[\begin{array}{ccc}
\sigma_{x}^{2} & 0 & 0 \\
0 & \sigma_{y}^{2} & 0 \\
0 & 0 & \sigma_{\theta}^{2}
\end{array}\right] \quad \phi(\tau)=\left[\begin{array}{ccc}
1 & 0 & -\frac{1}{2}\left(v_{R}+v_{L}\right) \tau \sin (\theta) \\
0 & 1 & \frac{1}{2}\left(v_{R}+v_{L}\right) \tau \cos (\theta) \\
0 & 0 & 1
\end{array}\right]
$$

Finally, after integration the final expression for the discrete process noise matrix is

$$
Q_{k}=\left[\begin{array}{ccc}
\sigma_{\theta} T_{s}^{3} v^{2} \sin (\theta)^{2}+\sigma_{x} T_{s} & -\left(T_{s}^{3} v^{2} \sigma_{\theta} \sin (2 \theta)\right) / 2 & -T_{s}^{2} v \sigma_{\theta} \sin (\theta) \\
-\left(T_{s}^{3} v^{2} \sigma_{\theta} \sin (2 \theta)\right) / 2 & \sigma_{\theta} T_{s}^{3} v^{2} \cos (\theta)^{2}+\sigma_{y} T_{s} & T_{s}^{2} v \sigma_{\theta} \cos (\theta) \\
-T_{s}^{2} v \sigma_{\theta} \sin (\theta) & T_{s}^{2} v \sigma_{\theta} \cos (\theta) & T_{s} \sigma_{\theta}
\end{array}\right]
$$

where $v=\frac{1}{2}\left(v_{R}+v_{L}\right)$ and $T_{s}$ represents the sample period. The process noise $\sigma_{x}, \sigma_{y}$, and $\sigma_{\theta}$ are updated every time stamp by Eqn.(4.11), (4.12), and (4.13). The camera system takes the measurement of state variables $X=[x, y, \theta]^{T}$ directly. Thus the measurement matrix is $H=I_{3}$.

The camera system takes the measurements of the state variables. Although the measurements contain some levels of noise, they are the closest values to true trajectory that we can get. As mentioned earlier, the noise of the camera system is relatively small. Furthermore, most of the measurement errors are produced by the distortion of the lens which has non-Gaussian distribution. In order to compare the performance of the filters with respect to the measurement errors with Gaussian 
distribution, we deliberately add white noise to the measurements. The standard

deviations of the noise are $\sigma_{x}=10, \sigma_{y}=10$ and $\sigma_{\theta}=\frac{5 \pi}{180}$. Finally, the EKF can be implemented by using Eqn.(2.58) to (2.62) recursively to compute the robot pose estimation.

\subsubsection{The UKF}

The implementation of the UKF is easier than the implementation of the EKF since it does not require any Jacobian derivations. The UKF is implemented simply following all the steps in section 2.6.2.

\subsubsection{The Bootstrap PF}

The bootstrap particle filter is performed by following Algorithm 2.10 for the robot pose estimation. The simulation is repeated by applying the bootstrap filter with sample sizes of 500, 1000, and 2500 respectively. For each sample size, 50 runs of Monte Carlo simulation are performed to gather sufficient data for statistical analysis.

\subsubsection{Initialization}

In the experiment, the robot was initially positioned at $(200,350)$ with orientation of 0 degree. Hence the initial state vector is

$$
x_{0}=\left(x_{0}, y_{0}, \theta_{0}\right)^{T}=(200,350,0)^{T}
$$

In each experiment, the robot moves along a circular path and travels from 0s to 20s. The measurements are taken by the camera set with the uniform sampling period of $T=0.1 \mathrm{~s}$. This means that each simulation trial consists 200 sample steps. All units in experiments are pixels in length and radian in angle. 
The initial covariance matrix $Q_{0}$ of the process noise is

$$
Q_{0}=\left[\begin{array}{ccc}
\sigma_{x}^{2} & 0 & 0 \\
0 & \sigma_{y}^{2} & 0 \\
0 & 0 & \sigma_{\theta}
\end{array}\right]=\left[\begin{array}{ccc}
3^{2} & 0 & 0 \\
0 & 3^{2} & 0 \\
0 & 0 & 1^{2}
\end{array}\right]
$$

Additional noise is added to the measurements to test the performance of each filter. Suppose that the measurement noise is additive white noise with Guassian distribution and $\eta_{x} \sim \mathcal{N}\left(0,10^{2}\right), \eta_{y} \sim \mathcal{N}\left(0,10^{2}\right)$, and $\eta_{\theta} \sim \mathcal{N}\left(0,\left(0,(5 \pi / 180)^{2}\right)\right.$. The covariance matrix $R$ of the measurement noise is

$$
R=\left[\begin{array}{ccc}
\eta_{x}^{2} & 0 & 0 \\
0 & \eta_{y}^{2} & 0 \\
0 & 0 & \eta_{\alpha}^{2}
\end{array}\right]=\left[\begin{array}{ccc}
10^{2} & 0 & 0 \\
0 & 10^{2} & 0 \\
0 & 0 & (5 \pi / 180)^{2}
\end{array}\right]
$$

The error covariance of initial pose estimation $P_{0}$ is

$$
P_{0}=\left[\begin{array}{ccc}
5^{2} & 0 & 0 \\
0 & 5^{2} & 0 \\
0 & 0 & (\pi / 180)^{2}
\end{array}\right]
$$

\subsection{Results}

The mobile robots are steered by spinning the wheels at different speeds with respect to one another. The robots are driven by the motors attached on its left and right wheels. The non-linear filters including the EKF, UKF and the bootstrap particle 
filter are applied to perform the pose estimate by using the system model and the measurement model described in section 4.5.

In experiments, the robots are programmed to move along a circular path. The input which controls the motors' speed is recorded by an interrupt handler every 0.1 second. Simultaneously, the robot's trajectories are recorded by the camera systems. The data that is collected in the experiment are post-processed by the filters to perform the pose estimate. The task of the filters is to estimate the system state $x_{k}=\left(x_{k}, y_{k}, \theta_{k}\right)^{T}$, where $x_{k}$ and $y_{k}$ specify the coordinates of the position of the robot in the Cartesian plane, the term $\theta_{k}$ denotes the angle between the longitude of the robot and the $x$-axis of the Cartesian plane. Finally, the pose estimates of each filter are compared with each other and compared with the trajectory that is recorded by the camera.

The mobile robot moves in a circular path in a clockwise direction as shown in Figure 4.5. However, the robot's real paths often deviate from the programmed paths in the experiments due to the process noise. Figure 4.6 and 4.7 illustrate RMSE of positions and headings for each non-linear filter.

As described earlier, the data is collected on-line from the robot control system and the measurement system in the experiments. The collected data is then processed off-line to perform the pose estimate by applying the non-linear filters. In order to compare the filter's performance on the pose estimation, some statistical methods are employed. 50 Monte Carlo trials were performed on each non-linear filter for pose estimation based on the experimental data. The RMSE of the performance of the filters is averaged and then compared. The average RMSE (the root mean square difference between the true values and the state estimates) are computed by 


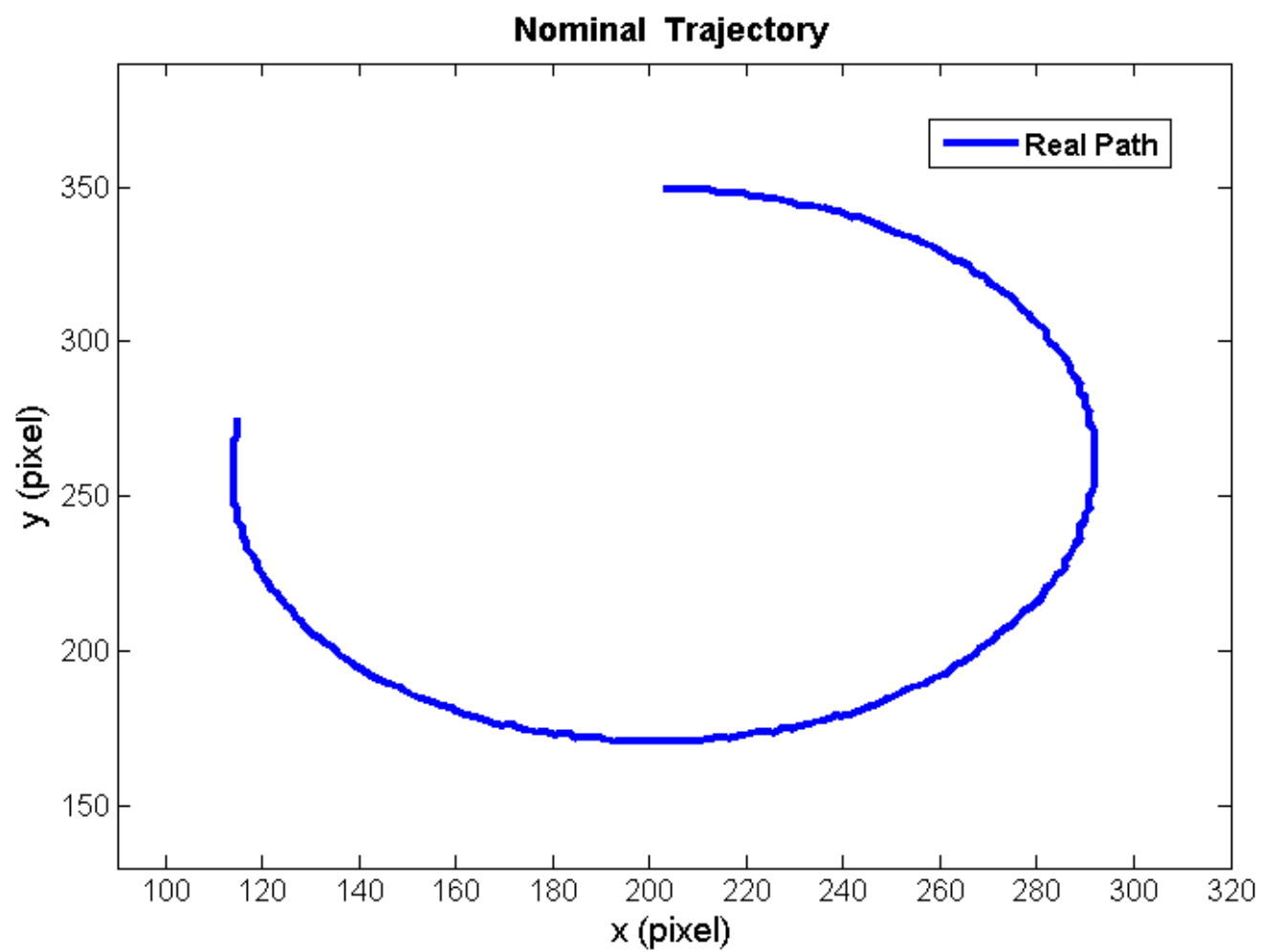

Figure 4.5: Robot Navigational Path Recorded by the Camera System(Solid line).

the following equation:

$$
R M S E=\frac{1}{n} \sum_{j=1}^{n} \sqrt{\frac{1}{N} \sum_{i=1}^{N}\left(x_{j}^{i}-\hat{x}_{j}^{i}\right)^{2}}
$$

where:

- $x_{j}^{i}$ specifies the true system state at the time step $j$ of $i$ th trial. $(j=1, \ldots, N$; $i=1, \ldots, n)$.

- $\hat{x}_{j}^{i}$ is the system state at the time step $j$ of $i$ th trial.

- System state index: $j=1, \ldots, N$,(In our case, $N=200)$.

- Monte Carlo trial index: $i=1, \ldots, n$, (In our case, $n=50$ ).

The average RMSE and its variance are evaluated and presented in Table 4.2 and 4.3. The computational complexity of each filter is illustrated in Table 4.4. The 


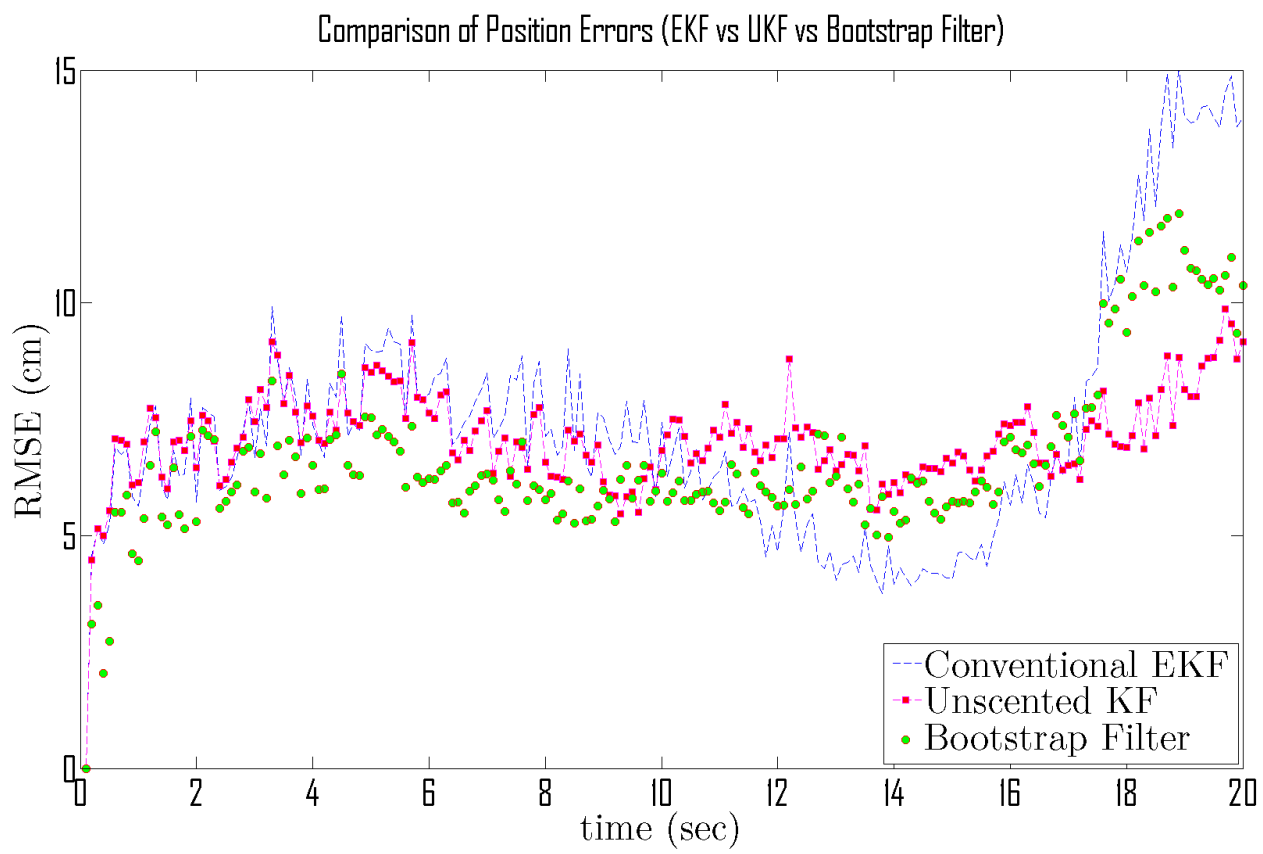

Figure 4.6: A comparison of RMS errors of position: EKF,UKF,Bootstrap Filter(2500 samples)

UKF and the bootstrap BF outperform the EKF based on the result of the RMSE comparison in Table 4.2. However, the EKF is still the most efficient algorithm. It takes only 0.18 seconds to complete the computation of the pose estimation. The UKF has a better performance than the EKF and the bootstrap filter (500 particles) on the state estimation. Although it takes about 3.4 seconds (18 times over the EKF) to implement the UKF algorithm, it is still much less than 20 seconds of the navigation time. To implement the pose estimation in our experimental platform, the $\mathrm{UKF}$ is a better choice than the EKF.

In the simulations of Chapter 3 , the bootstrap filter provides a much better performance over the EKF and UKF. However, based on the data of the experiments, it is clearly noticeable that the bootstrap filter can only provide a close or a slightly better performance over the UKF on pose estimation. The bootstrap filter (2500 particles) only outperforms about $5 \%$ over the UKF with respect to the RMSE of positions. To perform the pose estimate for a trial of 20 seconds, the bootstrap filter 


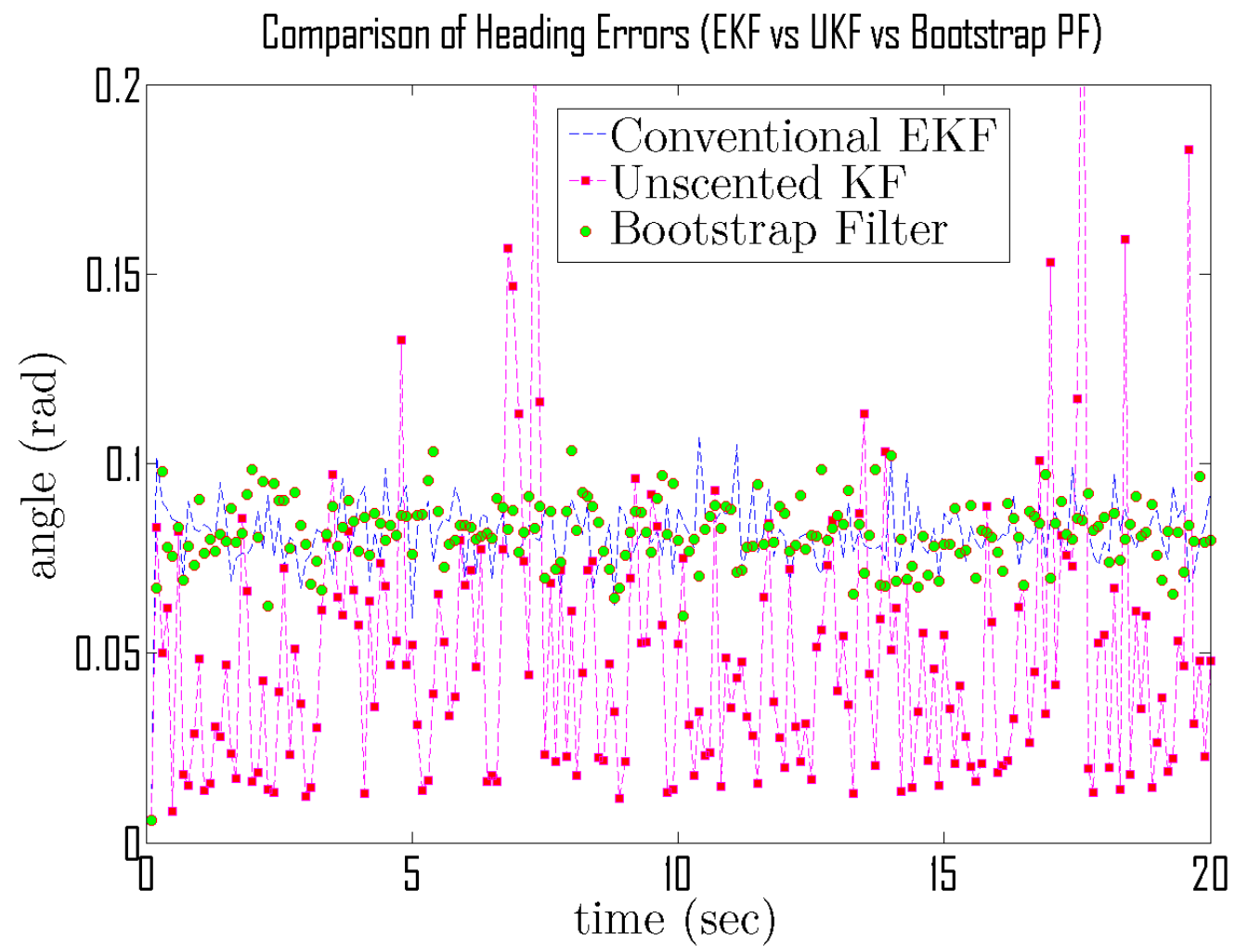

Figure 4.7: A comparison of RMS errors of heading: EKF,UKF,Bootstrap Filter(2500 samples)

Table 4.2: Time-Averaged RMS Position Error in Centimeters

\begin{tabular}{|c|c|c|}
\hline Algorithm & RMSE-Position & VAR \\
\hline EKF & 7.7979 & 1.2979 \\
\hline UKF & 7.1039 & 0.8222 \\
\hline Bootstrap Filter $(\mathrm{n}=500)$ & 7.1676 & 0.98542 \\
\hline Bootstrap Filter $(\mathrm{n}=1000)$ & 6.8544 & 1.0388 \\
\hline Bootstrap Filter $(\mathrm{n}=2500)$ & 6.8261 & 1.0168 \\
\hline
\end{tabular}

takes around 100 seconds to complete the computation of 500 particles. That is, the computational complexity of the bootstrap filter is too high to perform on-line pose estimation in the experiments.

Table 4.2 also indicates that an increase in the number of particles can reduce 


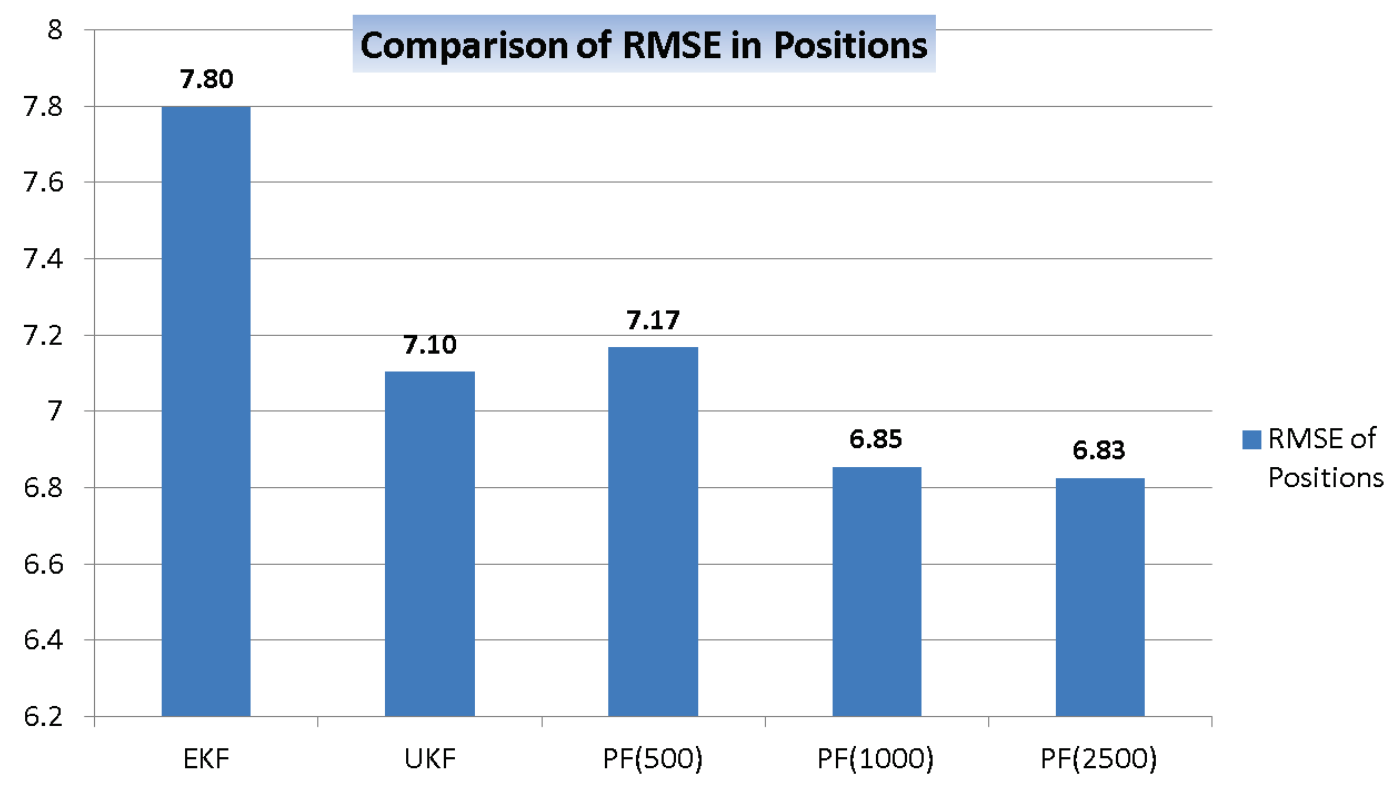

Figure 4.8: comparison of RMSE of position: EKF,UKF,Bootstrap Filter. (A illustration of data in Table 4.2)

Table 4.3: Time-Averaged RMS Heading Error in Centimeters

\begin{tabular}{|c|c|c|}
\hline Algorithm & RMSE-Heading & VAR \\
\hline EKF & 0.081901 & $1.4480 \mathrm{E}-5$ \\
\hline UKF & 0.062322 & $1.2456 \mathrm{E}-5$ \\
\hline Bootstrap Filter $(\mathrm{n}=500)$ & 0.081878 & $2.2368 \mathrm{E}-5$ \\
\hline Bootstrap Filter $(\mathrm{n}=1000)$ & 0.081309 & $1.4738 \mathrm{E}-5$ \\
\hline Bootstrap Filter $(\mathrm{n}=2500)$ & 0.081030 & $8.5285 \mathrm{E}-6$ \\
\hline
\end{tabular}

the RMSE for the bootstrap particle filter. However, the change in performance is negligible as the number of particle increases from $N=1000$ to 2500. Since the UKF has better performance than the EKF and much lower computational complexity than the particle filter, we finally conclude that the UKF is the best filter to perform the state estimation on our experimental platform.

Unlike the simulation in Chapter 3, we choose the UKF as the best non-linear filter 
Table 4.4: Computational Complexity

\begin{tabular}{|c|c|c|}
\hline Algorithm & Time(s) & Relative \\
\hline EKF & 0.1857 & 1.00 \\
\hline UKF & 3.3612 & 18.10 \\
\hline Bootstrap PF $(\mathrm{n}=500)$ & 98.1565 & 528.58 \\
\hline Bootstrap PF $(\mathrm{n}=1000)$ & 195.223 & 1051.3 \\
\hline Bootstrap PF $(\mathrm{n}=2500)$ & 486.416 & 2619.4 \\
\hline
\end{tabular}

for the experiments. We notice that the camera system measures the state variables directly. That is, the measurement system of the experimental platform has a linear model, whereas the measurement system has a non-linear model in the simulation of Chapter 3. This is the major difference between the modelling of the simulation and experimental platforms.

Meanwhile, there are many factors that contribute to producing the errors with Gaussian and non-Gaussian distribution in the experiments. These errors include, but are not limit to the process noise, observational noise and model definition errors. Since it is difficult to evaluate some of these errors precisely like the noise of the motors and the uneven wheels of the robot, the filters cannot achieve a better quality of pose estimation without including the effect of all these errors.

Based on the experimental result as shown in Table 4.2, the UKF and the bootstrap particle filter outperform the EKF on pose estimation. The UKF can theoretically capture the first and second order moments of the state distribution of the Gaussian random variables. It appears to achieve a better representation of the experimental non-linear system over the EKF. The particle filter (PF) can perform the pose estimate without being subject to any linearity or Gaussianity constraints of the systems. However, the particle filter' performance is only slightly better than the UKF. Moreover, it is difficult to apply the particle filter to real time pose estimation 
due to its prohibitive computational complexity.

Finally, we can conclude that it is not necessary to perform the pose estimate by using the particle filter. The UKF is the best choice to perform the pose estimate in the experiments.

\subsection{Chapter Summary}

This chapter started by introducing an experimental platform. The platform's architecture and components were explained in detail. The derivation of the model of the two-wheel mobile robot was also presented. The parameters and the detailed implementation methods for each non-linear filter were then described . The experimental data were collected on-line during the experiments. The collected data were then post-processed off-line for the pose estimation. Each filter was applied to perform the pose estimation based on the experimental data. The performance of the filters was compared with each other. The EKF was the most efficient approach with the least computational complexity among all the three filters. The UKF outperformed the EKF and the bootstrap particle filter (500 particles). It took the UKF less than 4 seconds to compute the pose estimation. Since the robot's navigation time was 20 seconds, the UKF can certainly be used to perform the real time pose estimate. The bootstrap filter had similar performance to the UKF. As discussed in Chapter 2, the particle filter had appealing performance on state estimation of highly non-linear systems, but it is often not practical for real time applications due to its prohibitive computational cost. In our case, the computational cost of the particle filter was much higher ( $>60$ times) than the EKF and the UKF. The bootstrap filter (2500 particles) only outperformed the UKF about 5\%. Obviously, it was not necessary to perform the pose estimation by the particle filter. Overall, the UKF was certainly the best choice in the experiments since it maintained the balance between the performance 
and the computational complexity. It was also a practical method for on-line pose estimation. 


\section{Chapter 5}

\section{Conclusion and Recommendations}

\subsection{Conclusion}

Mobile robot pose estimation is an ability to perceive the robot location and orientation of where it currently is in the environment in order to ensure it is on the proper course. An accurate pose estimate is essential for a mobile robot to successfully accomplish its tasks during navigation. The models of the mobile robots are generally introduced in a nonlinear fashion and corrupted by noise. Thus, nonlinear filters are required to eliminate system noise and accurately perform robot pose estimation. The goal of this research described in this thesis is to compare the performance of three popular nonlinear filters. The EKF, the UKF and the bootstrap PF were implemented to perform pose estimate in the simulation and experiments. The main metric used to determine filter's performance was RMSE of pose estimation and filter's computational complexity.

From the results in Chapter 3 it is clear that the EKF provided the worst performance comparing to the other two filters. The UKF had slightly better performance over the EKF in terms of RMSE for the pose estimation. However, its computational cost was over 10 times higher than the EKF. Obviously, the EKF was definitely a better choice over the UKF on robot pose estimation. 
The bootstrap filter had the lowest RMSE both on the estimation of position and orientation. But its computational complexity was too high to perform the real time pose estimate for robot navigation. The parallel computation and a high-level language such as $\mathrm{C}++$ are required when implementing the bootstrap PF for the real time pose estimation.

From the results of the experiments in Chapter 4 the EKF was the most efficient approach for pose estimation. The UKF also had more than 10 times computational complexity over the EKF. However, the time used to perform the pose estimate for both the EKF and the UKF was much less time the robot's navigation time. That is, both the EKF and the UKF can be used to perform the real time pose estimate. In our experiments, the computational cost of the particle filter was much higher $(>60$ times) than that of the EKF and the UKF. Clearly, the bootstrap PF was not a practical approach for real time pose estimation due to its prohibitive computational cost.

The results of the experiments demonstrate that the UKF had better performance than the EKF and the bootstrap particle filter (500 particles) in term of RMSE on pose estimation. The bootstrap filter (2,500 particles) had the lowest RMSE result over 50 Monte Carlo trials. However, the bootstrap filter(2,500 particles) performed only slightly better than the UKF. Overall, the UKF was certainly the best choice in the experiments since it maintained the balance between the performance and the computational complexity. It was also a practical method for on-line pose estimation.

The performance was also compared between the bootstrap filter with 500, 1000, 2500 particles. The results indicated that the average RMSE decreased as the number of samples increased. If the computational cost is not our primary concern, the bootstrap filter can be thought of as the best filter with the highest quality of pose estimation. However, if the computation cost is critical, the EKF or the UKF are the options to be considered since they also provide relatively good 
performance.

\subsection{Thesis Contribution}

In this thesis, the performance of the non-linear filters was compared quantitatively in terms of RMSE of pose estimation. The filters were applied to perform mobile robot pose estimate. The system models of the mobile robots in the simulation and experiments were defined and evaluated in a state-space form with a set of non-linear differential equations. In summary, this thesis has provided advancements to this area of research through the following contributions:

1. Development of simulated environment: implemented the simulated application for robot pose estimation. The simulated environment consisted of two main components: a simulated robot model moving along a desired path and an observational model measuring the polar coordinates of the robots with respect to three known landmark. The environment was developed in Matlab. The convenience of programming and debugging in MATLAB enabled this simulation engine to be developed quickly.

2. Conducting experiments: The robots in the experiment were modelled based on the experimental results and the manufacture standards. The measurement system was calibrated by the experiments. That is, the camera system was evaluated to determine the level of the measurement noise. In order to sample the data simultaneously from the camera system and the input to both motors that driven the robot's left and right wheels, the software both on the computer and on the robot were upgraded.

3. Development of non-linear filters: the filters including the EKF, UKF and several variants of the $\mathrm{PF}$ were implemented in Matlab and $\mathrm{C}++$ to perform pose 
estimate in the simulations and experiments.

4. Implementation of Square-Root filtering and factorization methods: the Bierman-Thornton UD filtering method is the most stable algorithm of the EKF. It was developed and used in the simulations and the experiments to decorrelate the measurement noise and improve the reliability of the algorithm.

5. Evaluation of the filters' performance: the non-linear filters were applied to perform robot pose estimate in the simulation and the experiments. 50 Monte Carlo trials were conducted with respect to each filter for the pose estimation. The results were compared in terms of RMSE of pose estimation and computational complexity. The best filter for pose estimation was finally determined with respect to the performance.

6. Evaluation of the performance of the particle filter of different numbers of particles: 50 Monte Carlo trials were conducted for the pose estimation by using the particle filter with 500, 1000, and 2500 particles respectively. The performance was compared and evaluated in terms of RMSE of pose estimation. The PF's computational complexity was also compared with each other.

\subsection{Recommendations}

Due to time constraints, certain trials or extensions of this work could not be accomplished or properly investigated. Some of them are listed here:

1. Model Definition: the model of the mobile robot in experiments has to be evaluated more precisely. For example, the motor output contains noise and the wheels of the robot are different from the manufacturer standards. In order to achieve a better quality of pose estimation, a more accurately defined model of the robot is required by including all kinds of noise. 
2. Filter Optimization: A more general problem yet to solved is that of optimizing all parameters associated with the filters. For instance, the UKF has $\alpha, \beta, \gamma$, $\mathrm{R}$ and $\mathrm{Q}$ that have to be set. Even just a set of rules to help determine these values would be useful.

3. Adaptive Particle Filter: In the future, it would be interesting to investigate the applications of pose estimation with the adaptive particle filter (APF). The APF aims at finding a balance point between the computational load and the required accuracy. It can adaptively adjust the number of required particles over time. It would be interesting to compare the performance of the APF with the data of the three filters in this thesis.

4. Parallel Computation: In order to implement the PF for real time pose estimation. An optimization of the PF implementation is required. One option is to use GPUs(Graphics processing unit). The GPUs consist of thousands of smaller, more efficient cores designed for parallel performance.

5. PF Implementation on $\mathrm{C}++$ : Another option to mitigate the problem of the computational complexity of the PF is to implement the algorithm with a high level language like $\mathrm{C}++$. 


\section{List of References}

[1] C.-F. Lin. Modern navigation, guidance, and control processing, volume 2. Prentice Hall Englewood Cliffs (1991).

[2] A. Doucet, N. De Freitas, and N. Gordon. An introduction to sequential Monte Carlo methods. Springer (2001).

[3] A. Gelb. Applied optimal estimation, pages 180-228. MIT press (1974).

[4] U. Henning, J. Timmer, and J. Kurths. "Nonlinear dynamical system identification from uncertain and indirect measurements." International Journal of Bifurcation and Chaos 14(06), 1905-1933 (2004).

[5] S. J. Julier and J. K. Uhlmann. "A new extension of the kalman filter to nonlinear systems." In "AeroSense'97," pages 182-193. International Society for Optics and Photonics (1997).

[6] S. J. Julierll and J. K. Uhlmann. "Unscented filtering and nonlinear estimation." Proceedings of the IEEE 92(3), 401-422 (2004).

[7] S. Julier, J. Uhlmann, and H. F. Durrant-Whyte. "A new method for the nonlinear transformation of means and covariances in filters and estimators." Automatic Control, IEEE Transactions on 45(3), 477-482 (2000).

[8] E. A. Wan and R. Van Der Merwe. "The unscented kalman filter for nonlinear estimation." In "Adaptive Systems for Signal Processing, Communications, and Control Symposium 2000. AS-SPCC. The IEEE 2000," pages 153-158. IEEE (2000).

[9] N. Metropolis and S. Ulam. "The monte carlo method." Journal of the American statistical association 44(247), 335-341 (1949).

[10] N. J. Gordon, D. J. Salmond, and A. F. Smith. "Novel approach to nonlinear/non-gaussian bayesian state estimation." In "Radar and Signal Processing, IEE Proceedings F," volume 140, pages 107-113. IET (1993). 
[11] A. Saberi, A. A. Stoorvogel, and P. Sannuti. Filtering theory: with applications to fault detection, isolation, and estimation. Birkhäuser Boston (2006).

[12] H. Sorenson. "Least-squares estimation: from gauss to kalman." Spectrum, IEEE 7(7), 63-68. ISSN 0018-9235 (1970).

[13] C. F. Gauss. Theoria Motus Corporvm Coelestivm In Sectionibvs Conicis Solem Ambientivm. Perthes et Besser (1809).

[14] A. M. Legendre. Nouvelles méthodes pour la détermination des orbites des cometes. F. Didot (1805).

[15] A. Hald. "TN Thiele's contributions to statistics." International Statistical Review/Revue Internationale de Statistique pages 1-20 (1981).

[16] S. L. Lauritzen. "Time series analysis in 1880: A discussion of contributions made by TN Thiele." International Statistical Review/Revue Internationale de Statistique pages 319-331 (1981).

[17] D. Simon. Optimal state estimation: Kalman, $H$ infinity, and nonlinear approaches. Wiley-Interscience (2006).

[18] A. N. Kolmogorov, W. Doyle, and I. Selin. "Interpolation and extrapolation of stationary random sequences." (1962).

[19] N. Wiener. Extrapolation, interpolation, and smoothing of stationary time series: with engineering applications. Technology Press of the Massachusetts Institute of Technology (1950).

[20] R. E. Kalman et al. "A new approach to linear filtering and prediction problems." Journal of basic Engineering 82(1), 35-45 (1960).

[21] R. E. Kalman and R. S. Bucy. "New results in linear filtering and prediction theory." Journal of basic Engineering 83(3), 95-108 (1961).

[22] B. A. McElhoe. "An assessment of the navigation and course corrections for a manned flyby of mars or venus." Aerospace and Electronic Systems, IEEE Transactions on (4), 613-623 (1966).

[23] G. L. Smith, S. F. Schmidt, and L. A. McGee. Application of statistical filter theory to the optimal estimation of position and velocity on board a circumlunar vehicle. National Aeronautics and Space Administration (1962). 
[24] P. Fearnhead. "Using random quasi-monte-carlo within particle filters, with application to financial time series." Journal of Computational and Graphical Statistics 14(4), 751-769 (2005).

[25] M. Klaas, N. De Freitas, and A. Doucet. "Toward practical n2 monte carlo: The marginal particle filter." arXiv preprint arXiv:120\%.1396 (2012).

[26] M. K. Pitt and N. Shephard. "Filtering via simulation: Auxiliary particle filters." Journal of the American statistical association 94(446), 590-599 (1999).

[27] M. S. Arulampalam, S. Maskell, N. Gordon, and T. Clapp. "A tutorial on particle filters for online nonlinear/non-gaussian bayesian tracking." Signal Processing, IEEE Transactions on 50(2), 174-188 (2002).

[28] J. S. Liu and R. Chen. "Sequential monte carlo methods for dynamic systems." Journal of the American statistical association 93(443), 1032-1044 (1998).

[29] T. Söderström and P. Stoica. System identification. Prentice-Hall, Inc. (1988).

[30] L. Ljung. System identification: theory for the user. Prentice-Hall, Inc., Upper Saddle River, NJ, USA. ISBN 0-138-81640-9 (1986).

[31] P. Zarchan and H. Musoff. Fundamentals of Kalman filtering: a practical approach, volume 208. Aiaa (2005).

[32] G. Bishop and G. Welch. "An introduction to the kalman filter." Proc of SIGGRAPH, Course 8, 27599-3175 (2001).

[33] S. F. Schmidt. "The kalman filter-its recognition and development for aerospace applications." Journal of Guidance, Control, and Dynamics 4(1), 4-7 (1981).

[34] S. J. Julier. "The scaled unscented transformation." In "American Control Conference, 2002. Proceedings of the 2002," volume 6, pages 4555-4559. IEEE (2002).

[35] G. Bierman. "A comparison of discrete linear filtering algorithms." Aerospace and Electronic Systems, IEEE Transactions on AES-9(1), 28-37. ISSN 00189251 (1973).

[36] G. Bierman. "Factorization methods for discrete sequential estimation." Mathematics in science and engineering ( 128 (1977).

[37] M. S. Grewal and A. P. Andrews. Kalman filtering: theory and practice using MATLAB. Wiley-IEEE press (2011). 
[38] P. Dyer and S. McReynolds. "Extension of square-root filtering to include process noise." Journal of Optimization Theory and Applications 3(6), 444-458 (1969).

[39] F. Daum. "Nonlinear filters: beyond the kalman filter." Aerospace and Electronic Systems Magazine, IEEE 20(8), 57-69. ISSN 0885-8985 (2005).

[40] R. Van Der Merwe, A. Doucet, N. De Freitas, and E. Wan. "The unscented particle filter." Advances in neural information processing systems pages 584590 (2001).

[41] J. V. Candy. Bayesian Signal Processing: Classical, Modern and Particle Filtering Methods, volume 54. Wiley-Interscience (2011).

[42] B. Ristic, S. Arulampalam, and N. Gordon. Beyond the Kalman filter: Particle filters for tracking applications. Artech House Publishers (2004).

[43] C. Samson. "Time-varying feedback stabilization of car-like wheeled mobile robots." The International journal of robotics research 12(1), 55-64 (1993).

[44] C. Samson, K. Ait-Abderrahim, et al. "Mobile robot control. part 1: Feedback control of nonholonomic wheeled cart in cartesian space." (1990).

[45] Arduino. "Arduino - introduction." (2012).

[46] G. Welch and G. Bishop. "An introduction to the kalman filter." (1995).

[47] G. Kittagawa. "non-gaussian state-space modelling of non-stationary time series (with discussion)." J. Amer. Statistical Assoc 82, 1032-1063 (1987).

[48] B. P. Carlin, N. G. Polson, and D. S. Stoffer. "A monte carlo approach to nonnormal and nonlinear state-space modeling." Journal of the American Statistical Association 87(418), 493-500 (1992). 


\section{Appendix A}

\section{Filter Applications}

\section{A.1 Sample Application for the Kalman Filter}

A simple application is presented here to provide a better explanation for the operation and features of the the Kalman filter. This sample application is motivated by Greg Welch and Gary Bishop's paper: "An Introduction to the Kalman Filter" [46]. I implemented this sample in Matlab to provide references in my thesis.

\section{A.1.1 The System Models}

Suppose that we would like to estimate a scalar constant from noisy measurements. Given that we can take measurements of a voltage, the measurements are not accurate but corrupted by white noise with standard deviation of 0.1 volt. The system model is linear, thus it can be expressed by a linear difference equation:

$$
\begin{aligned}
x_{k} & =\phi_{k-1} x_{k-1}+u_{k-1}+w_{k} \\
& =x_{k-1}+w_{k}
\end{aligned}
$$


and the measurements $z \in \mathcal{R}^{1}$ are obtained from the measurement model which has the form:

$$
z_{k}=H x_{k}+\eta_{k}=x_{k}+\eta_{k}
$$

where the system state is a constant, that is, it does not change between steps so $\phi=1$. The control input $u=0$. The noisy measurements are of the state directly so $H=1$.

\section{A.1.2 The Kalman Filter and Parameters}

The Kalman filter algorithm is rewritten with respect to the system and measurement models. The propagation equations are rewritten as:

$$
\begin{aligned}
\hat{x}_{k}^{-} & =\hat{x}_{k-1}^{-} \\
P_{k}^{-} & =P_{k-1}+Q
\end{aligned}
$$

and the update equations are

$$
\begin{array}{r}
K_{k}=P_{k}^{-}\left(P_{k}^{-}+R\right)^{-1} \\
\hat{x}_{k}=\hat{x}_{k}^{-}+K_{k}\left(z_{k}-\hat{x}_{k}^{-}\right) \\
P_{k}=\left(1-K_{k}\right) P_{k}^{-}
\end{array}
$$

Let us set a very small process noise, $Q=1 e-5$. Also we know that the distribution of the measurements is a Gaussian random variable. We set initial state estimate $\hat{x}_{0}=0$.

Since the initial state estimate $\hat{x}_{0}$ is not accurate, the initial value for the error covariance $P_{0}$ can be any value but 0 to make the filter converge eventually. So we have $P_{0}=1$. 


\section{A.1.3 Simulations and Results}

The voltage, a scalar constant is chosen randomly: $x=-0.37727$. Simulations are conducted by 50 distinct measurements $z_{k}$. The measurement $z_{k}$ is disturbed by a noise which is a zero-mean normally distributed variable with a standard deviation of 0.1. A set of measurements has been saved and used for several simulations. The results are then compared between the simulations with different parameters.

In the first simulation, the measurement variance is set at $R=(0.1)^{2}=0.01$. Figure A.1 illustrate the results of the first simulation.

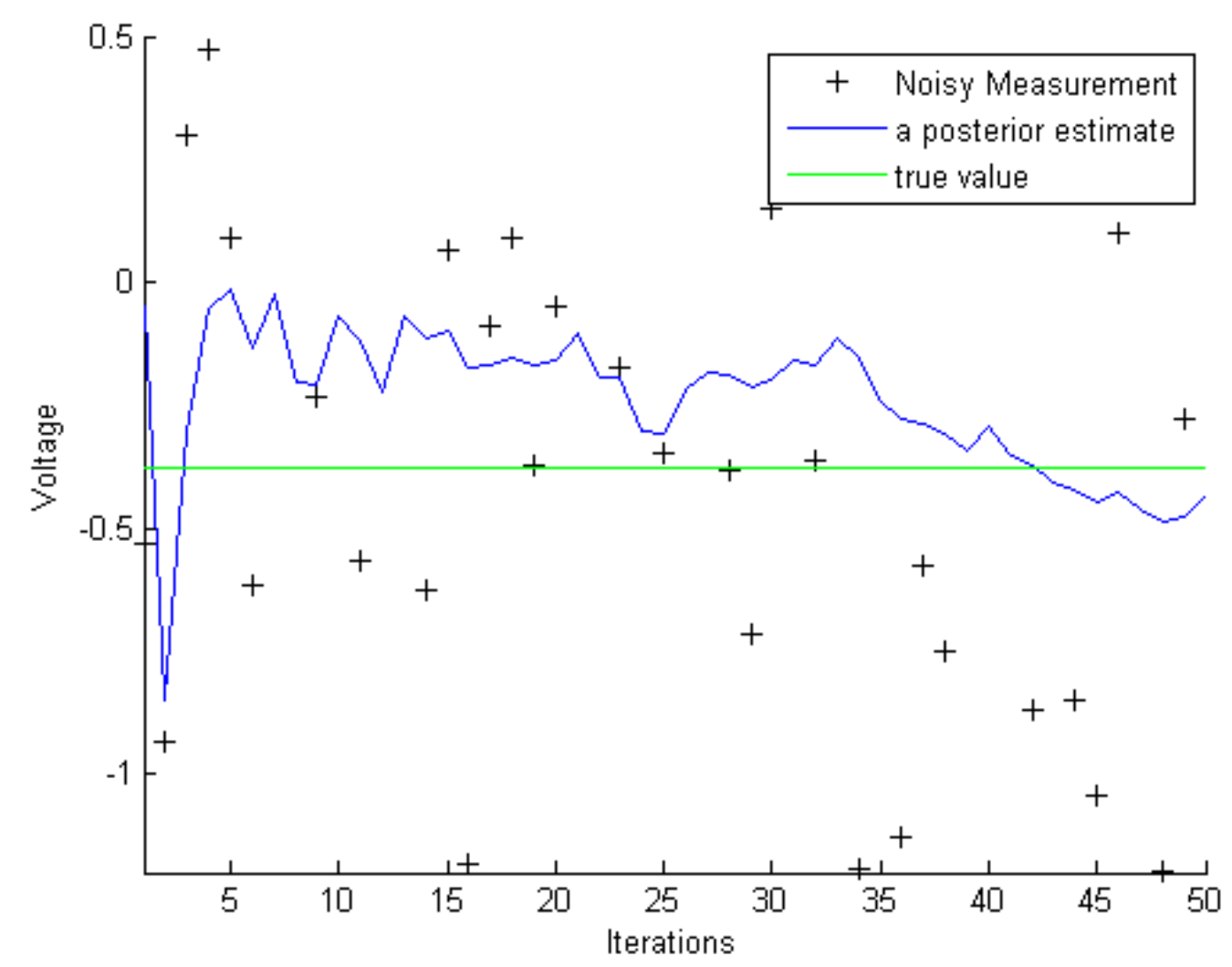

Figure A.1: The first simulation: $R=0.1^{2}=0.01$. The random constant $x=$ -0.37727 is depicted by the solid line, the noisy measurement is given by the cross marks, and the filter estimate by the remaining curve.

Since the filter converges eventually, it indicates that $P_{k}$ is approaching to zero 
after the iterations. Figure A.2 shows the value of $P_{k}$ changing along the iterations. After the $50^{\text {th }}$ iteration, it has changed from the initial value of 1 to approximately 0.0003 .

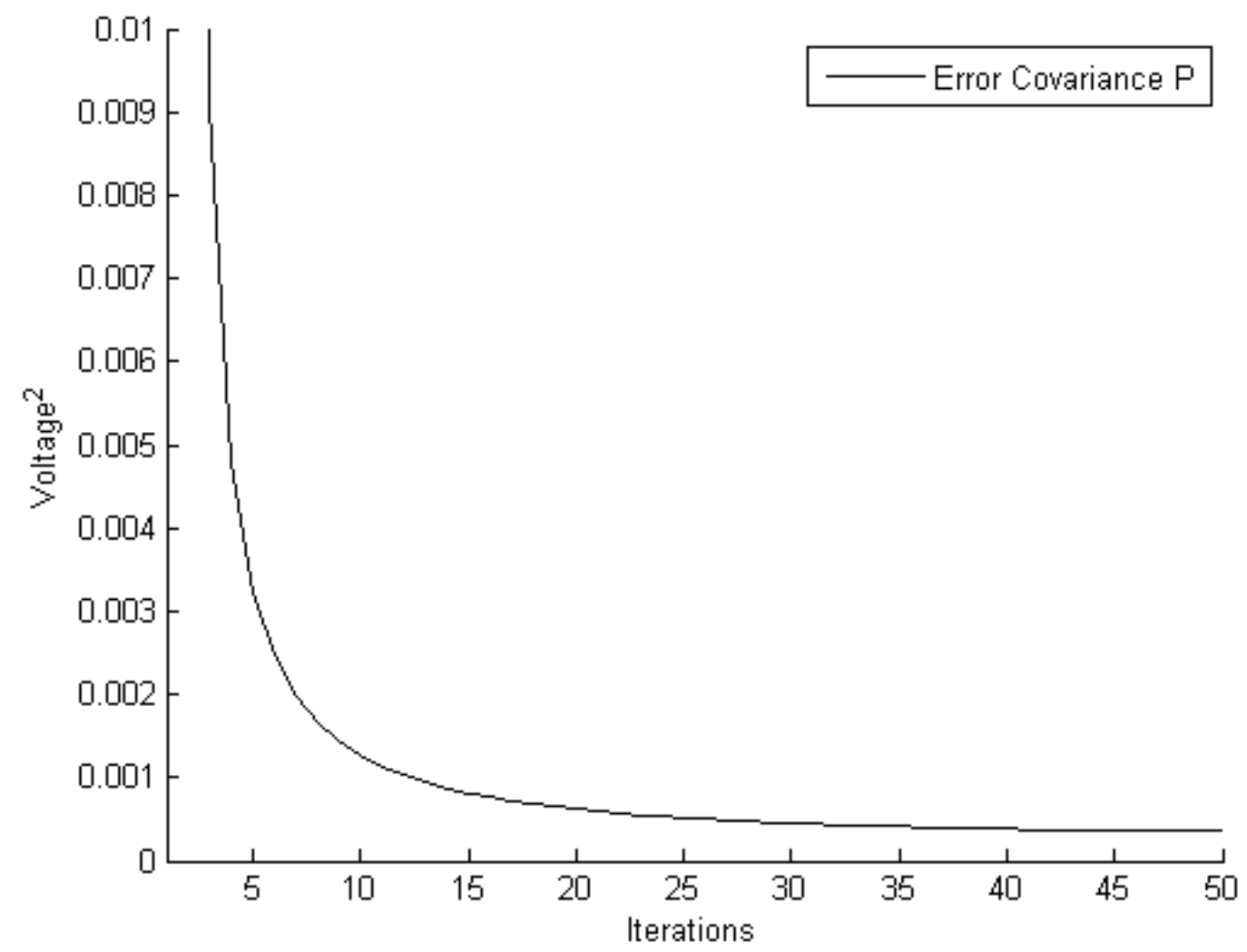

Figure A.2: By the $50^{\text {th }}$ iteration, the error covariance $P_{k}$ has settled from 1 to 0.0003

We conduct two more simulations based on the different parameters $Q$ and $R$. The covariance $R$ is increased or decreased by a factor of 100 respectively. The different performance indicates that $R$ can be a factor affecting the speed of filter's convergence. In Figure A.3, the measurement variance was 100 times greater (i.e. $R=1$ ). The filter converges slower but it has a smoother performance.

In Figure A.4, the measurement variance is set 100 times smaller(i.e. $\mathrm{R}=0.0001$ ). The filter tends to trust the noisy measurement so it has a larger variance. 


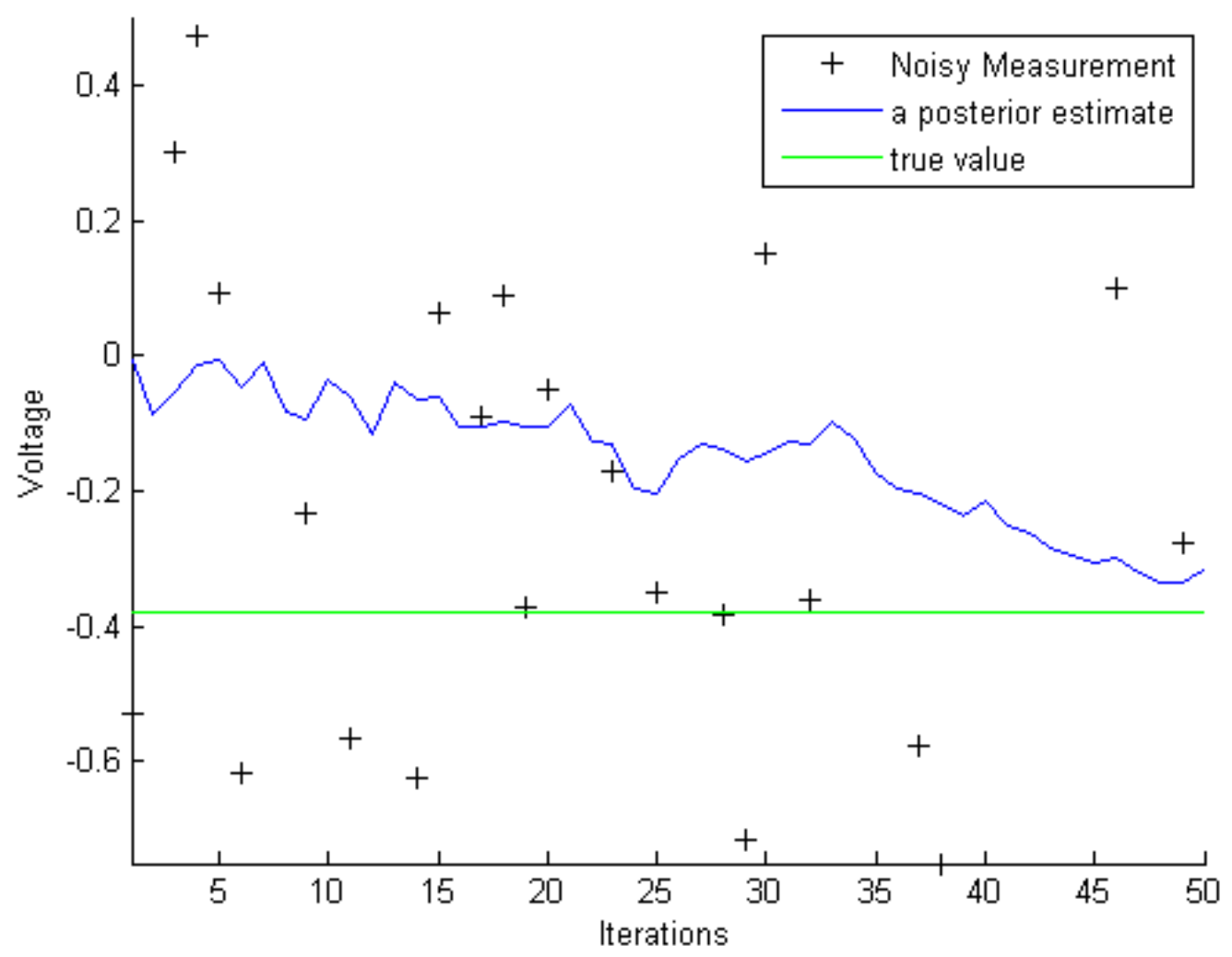

Figure A.3: Simulation: $R=1$. The filter is slower in convergence, resulting from a reduced estimate variance.

This example clearly demonstrates the nature of the Kalman filter. The estimates by the Kalman filtering approach in Figure A.3 appears considerably smoother and it has a reduced estimate variance.

\section{A.2 Sample Application for the EKF, the UKF and the PF}

We present an application to demonstrate the robustness of the non-linear filters when they are applied to highly non-linear and non-Gaussian systems. The sample application is a univariate non-stationary growth model which has been used extensively in 


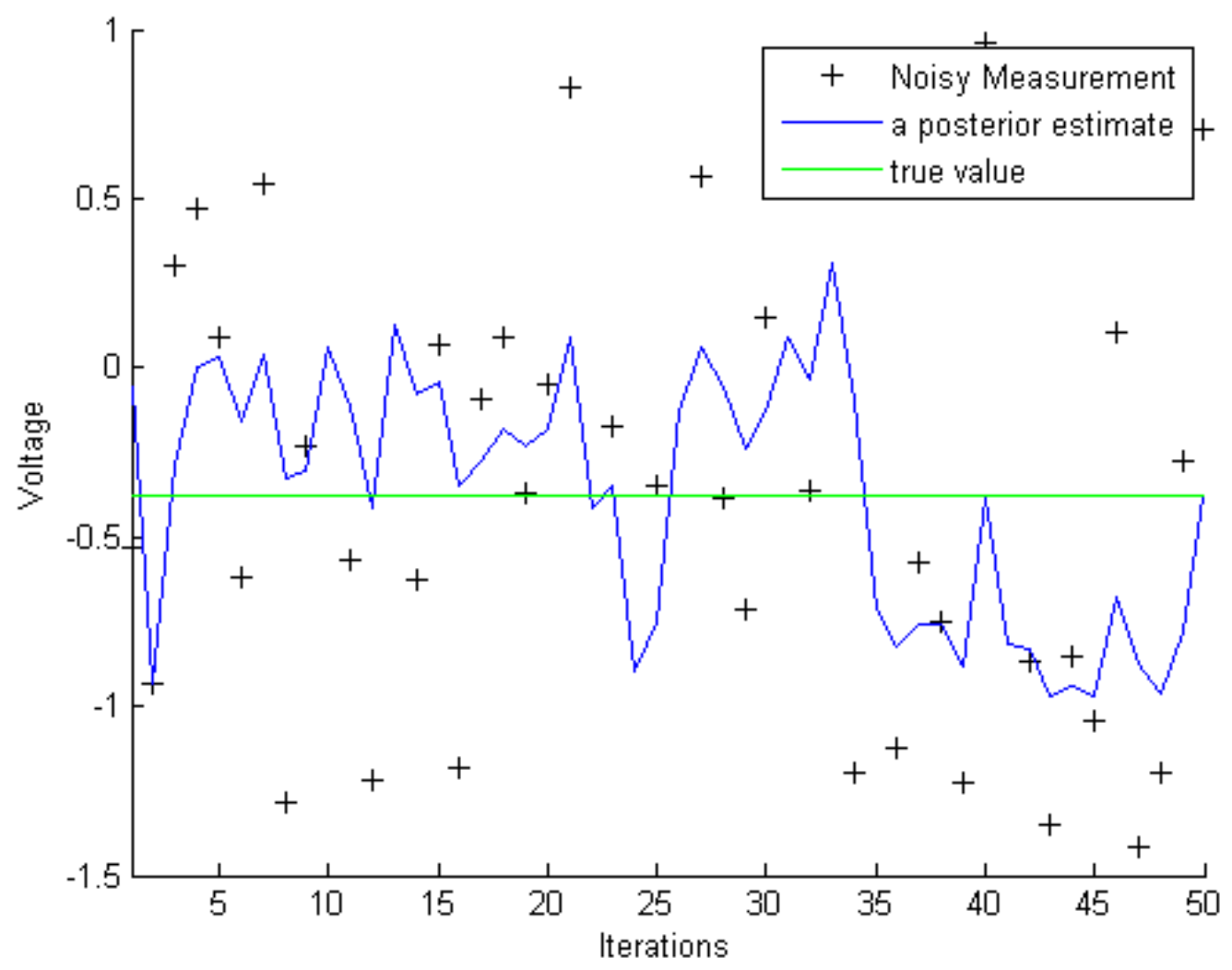

Figure A.4: Simulation: $R=0.00011$. The filter has a noisy performance, resulting from the increased estimate variance.

literature such as $[10,27,47,48]$. By comparing the performance of the filters including the EKF, UKF and the bootstrap PF, their capacity of dealing with non-linearity and non-Gaussianity are investigated. This example is implemented in Matlab to provide extra references in my thesis.

\section{A.2.1 One dimensional non-linear model and parameters}

We choose this model since it is highly non-linear, both in the system and the measurement functions. The dynamic state space equations for this model can be written 
as

$$
\begin{aligned}
& x_{n}=0.5 x_{n-1}+\frac{25 x_{n-1}}{1+x_{n-1}^{2}}+8 \cos (1.2(n-1))+w_{k} \\
& z_{k}=\frac{x_{n}^{2}}{20}+\eta, n=1, \ldots, N
\end{aligned}
$$

where $w_{k}$ and $\eta_{k}$ are zero-mean Gaussian with variance 10 and 1 . All filters were initialized with the prior $\operatorname{PDF} p\left(x_{0}\right)=\mathcal{N}(0,1)$ and the initial state $x_{0}=0.1$. Note that term $8 \cos (1.2(n-1))$ in the system dynamic model can be interpreted as timevarying noise since it is independent of $x_{n}$ but varies with time step $n$. Another interesting point is that the likelihood $p\left(z_{n} \mid x_{n}\right)$ has bimodal nature. For measurement $z_{n}<0$ the likelihood is unimodal. However, the likelihood is bimodal when $z_{n}>0$.

\section{A.2.2 Simulation Results}

Figure A.5 shows the true states of a 100 step realization of Eqn.(A.8). The estimation performance of the EKF, UKF and the bootstrap PF are compared based on the following metrics:

$$
R M S E_{\hat{x}}=\sqrt{\frac{1}{N} \sum_{n=1}^{N}\left(x_{n}-\hat{x}_{n}\right)^{2}}
$$

where $\hat{x}=E\left(x_{n} \mid z_{0: n}\right)$, and $R M S E$ is the root mean square error computed from the posterior state distribution.

The EKF is a first-order approximation of posterior Gaussian state distribution. It is not a sufficient description of the non-linear and non-Gaussian nature of the example. As shown in Figure A.6, the estimation performance of the EKF is very poor. The mean of the filter is rarely close to the true state. The implication of this is that the local linearization of the EKF is not an adequate approach to approximate the posterior density of the underlying modal.

The RMSE measure also indicates that the EKF is the least accurate of the filters 


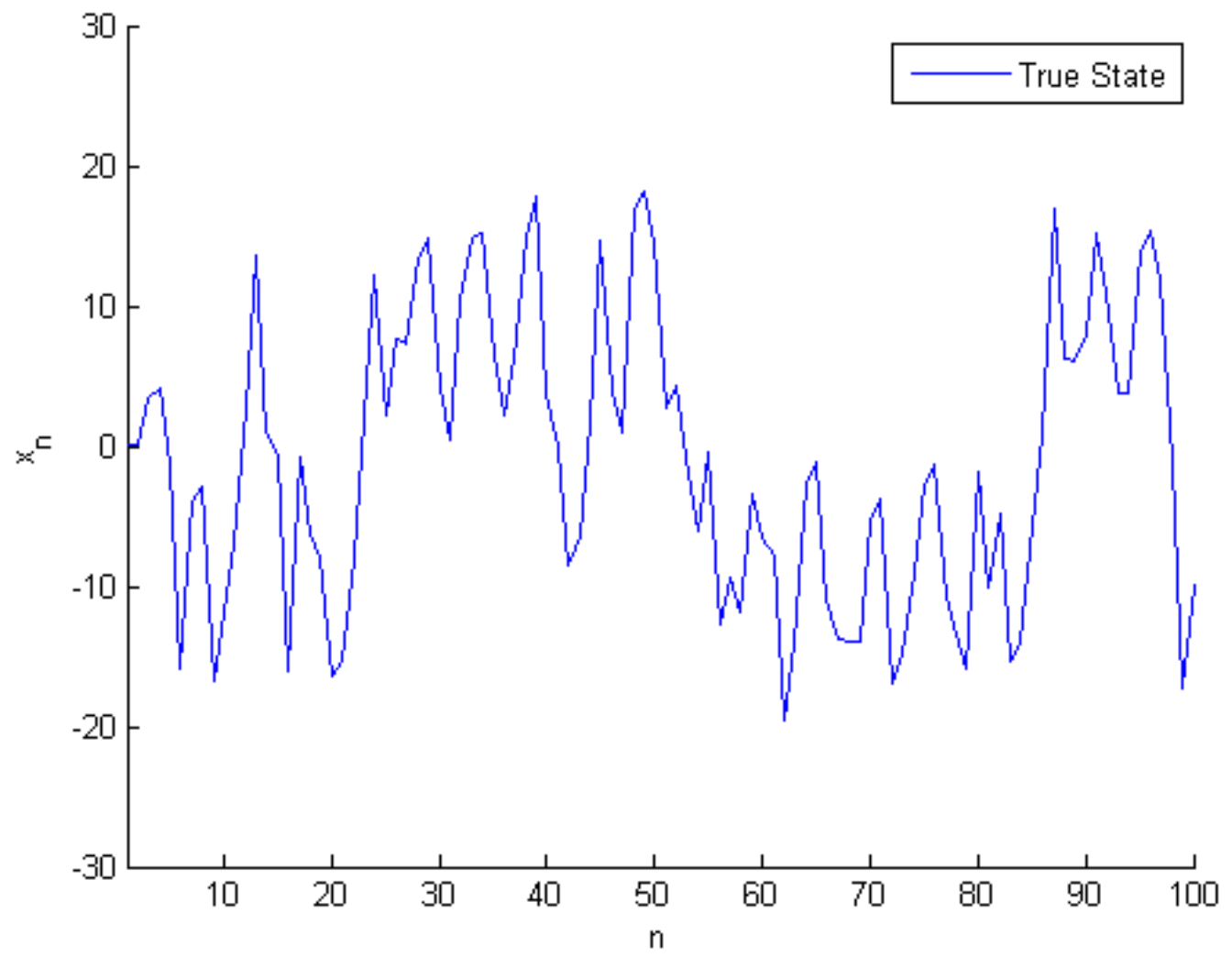

Figure A.5: Plot of the true states $x_{n}$ of 100 step realization

at approximating the posterior density. The EKF is not an adequate approach to approximate the highly non-linear and non-Gaussian density of the example.

The UKF algorithm can capture the first and second order moments of the state distribution of a Gaussian Random Variable $w_{k}[6]$. According to the comparison of RMSE, the UKF outperforms the EKF at approximating the highly non-linear system.

The particle filter (PF) has appealing convergence properties without being subject to any linearity or Gaussianity constraints. The bootstrap algorithm 2.10 is applied to the non-linear model. Figure A.8 shows the result of a realization of the bootstrap filter with 500 particles. Clearly, the bootstrap particle filter outperforms the EKF and UKF significantly for this highly non-linear system. Figure A.9 shows 


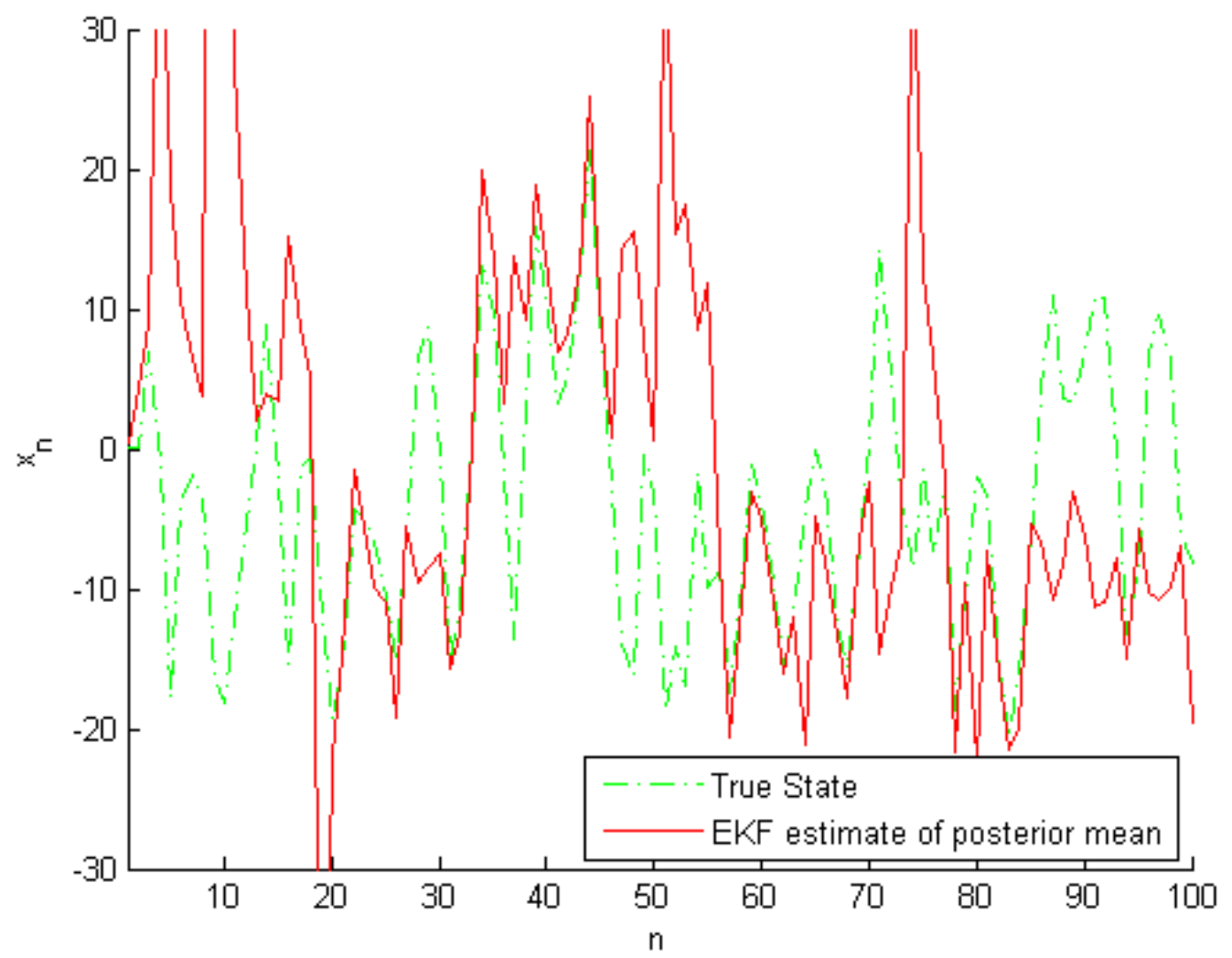

Figure A.6: Plot of the true states $x_{n}$ and the estimate of the EKF

the average RMSE for 50 random realizations with particle $N=100,500$, and 1000 . Even for a small number of particles $M=100$, the bootstrap particle filter performs better than the EKF and UKF. An increase in the number of particles can reduce the RMSE even further for the particle filter. However, the change in performance is negligible as the number of particle increases from $N=500$ to 1000 . 


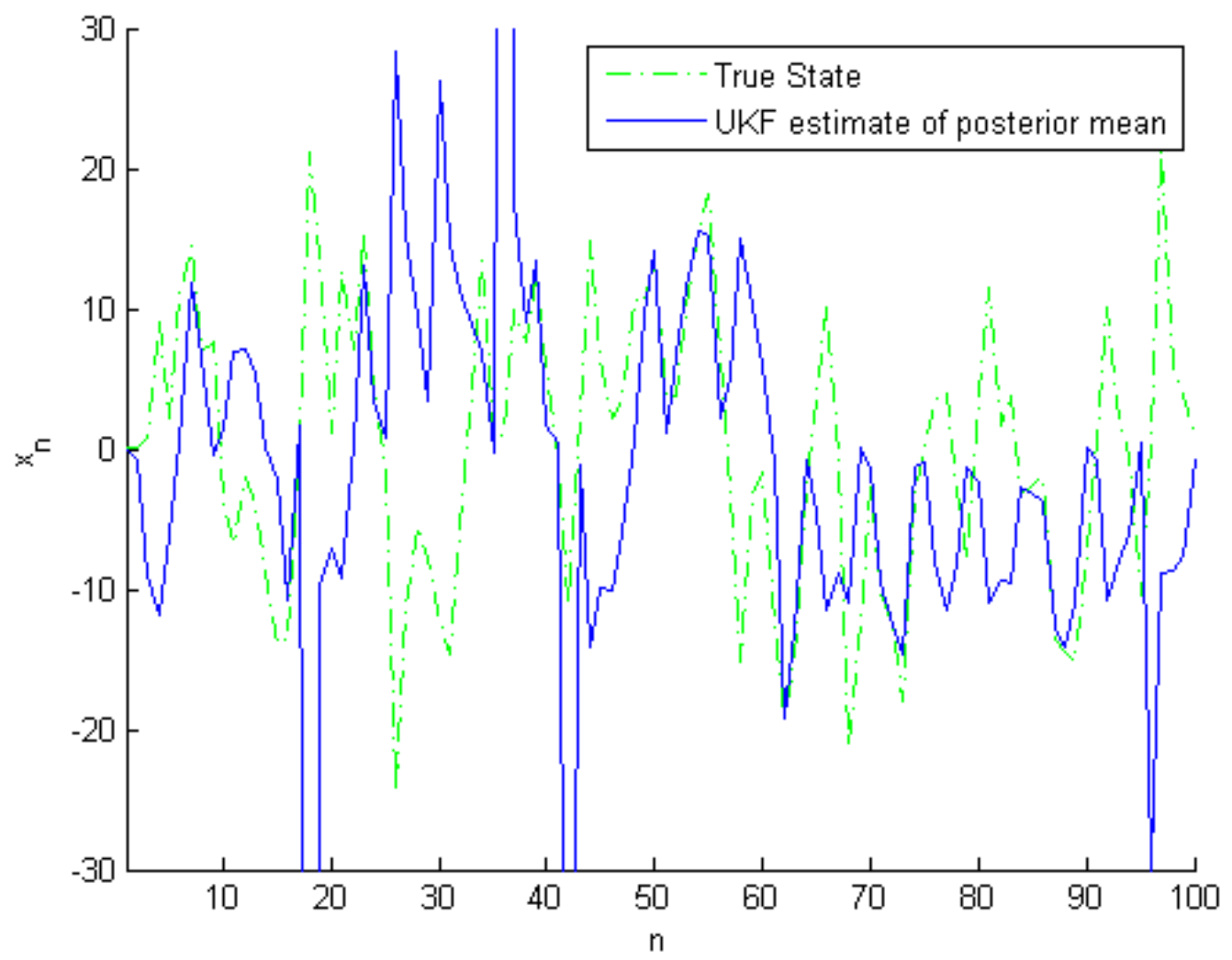

Figure A.7: Plot of the true states $x_{n}$ and the estimate of the UKF 


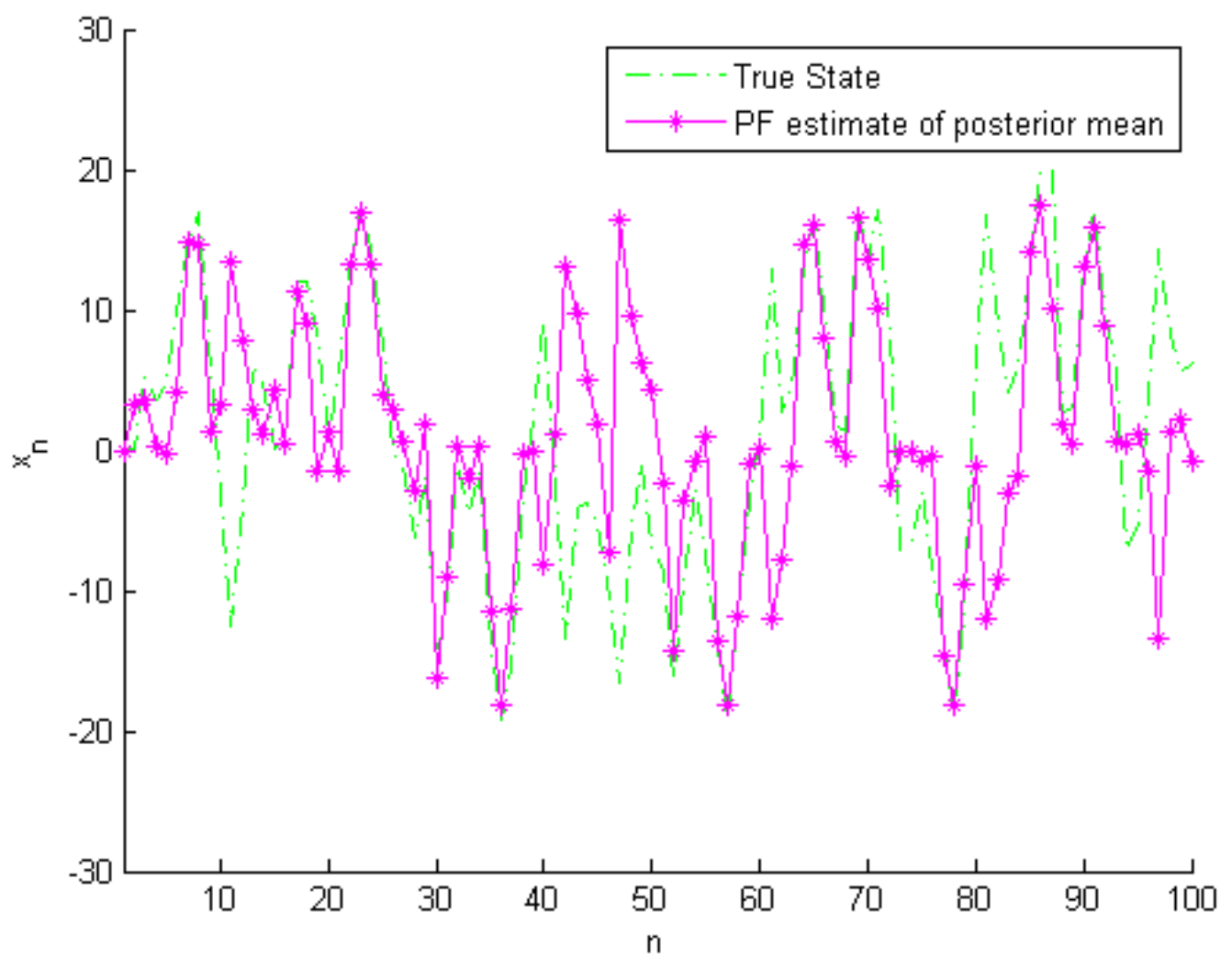

Figure A.8: Plot of the true states $x_{n}$ and the estimate of the PF (N=500) 


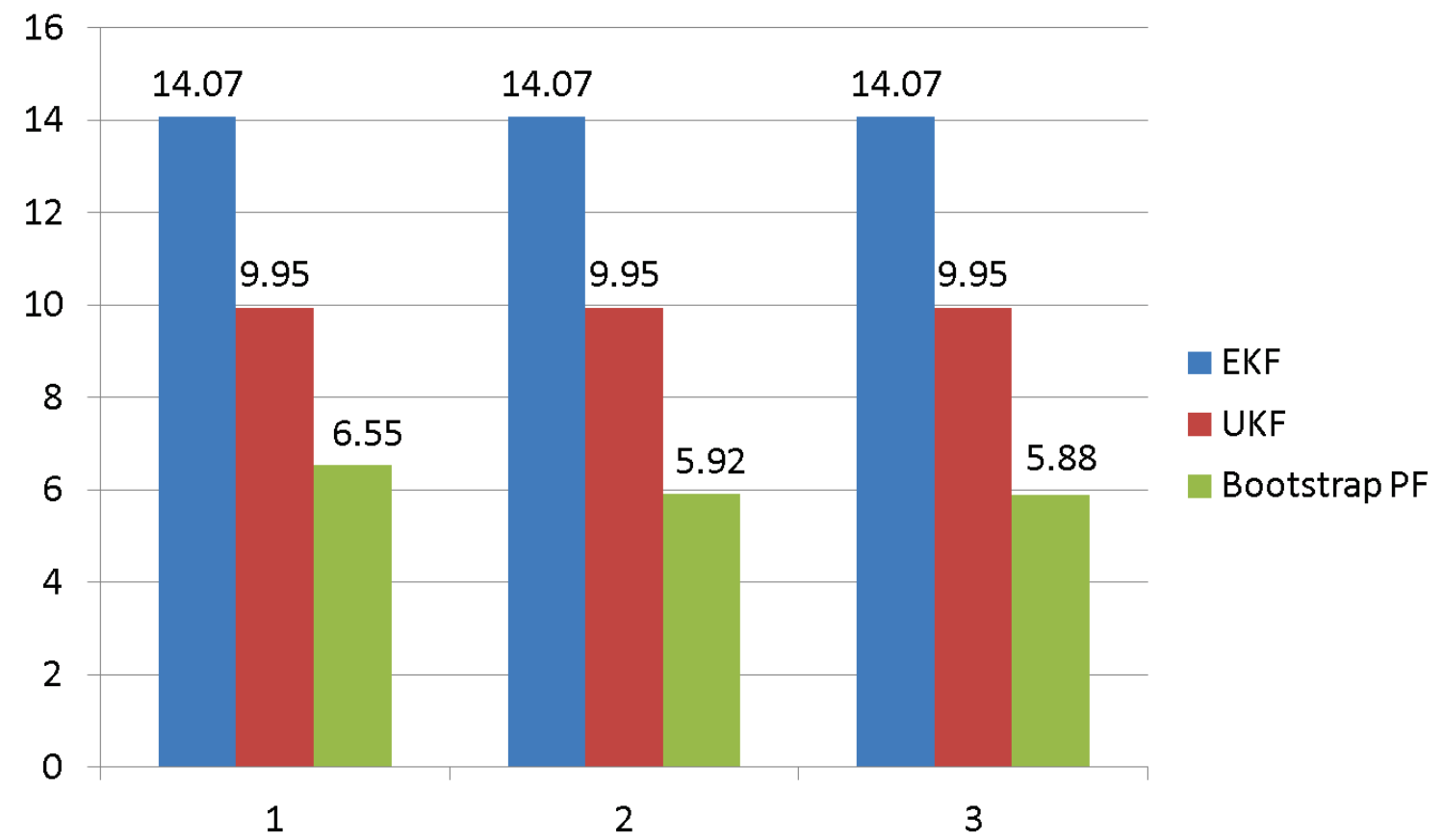

Figure A.9: Performance comparison of the EKF, UKF and the bootstrap PF $(\mathrm{N}=100,500$, and 1000$)$ 